\title{
Advanced Controllers for Electromechanical Motion Systems
}


Graduation committee:

$\begin{array}{lll}\text { Chairman: } & \text { prof.dr.ir. A.J. Mouthaan } & \text { University of Twente, EWI } \\ \text { Secretary: } & \text { prof.dr.ir. A.J. Mouthaan } & \text { University of Twente, EWI } \\ \text { Promotor: } & \text { prof.dr.ir. J. van Amerongen } & \text { University of Twente, EWI } \\ \text { Asst. promotor: } & \text { dr.ir. T.J.A. de Vries } & \text { University of Twente, EWI } \\ \text { Members: } & \text { prof.dr.ir. P.H. Veltink } & \text { University of Twente, EWI } \\ & \text { dr. J.W. Polderman } & \text { University of Twente, EWI } \\ & \text { prof.dr.ir. P.P.J. van den Bosch } & \text { University of Eindhoven } \\ & \text { prof.dr.ir. G. van Straten } & \text { University of Wageningen }\end{array}$

\section{disc}

The author has followed the educational program of the Dutch Institute of Systems and Control (DISC).

This research was financially supported by the Vietnamese Government through the 322 project and carried out at the Control Engineering group, University of Twente, The Netherlands.

ISBN: 978-90-365-2654-8

The cover figure of this thesis gives an overview of the input reference and the tracking errors for the different controllers that have been tested on the Tripod.

Copyright (C) 2008 by Nguyen Duy Cuong

Printed at Wöhrmann Print Service, Zutphen, The Netherlands. 


\section{ADVANCED CONTROLLERS \\ FOR \\ ELECTROMECHANICAL MOTION SYSTEMS}

\section{DISSERTATION}

to obtain

the degree of doctor at the University of Twente, on the authority of the rector magnificus, prof.dr.W.H.M. Zijm,

on account of the decision of the graduation committee, to be publicly defended on Thursday, $10^{t h}$ of April 2008 at $13.15 \mathrm{~h}$

by

Nguyen Duy Cuong born on $9^{\text {th }}$ of May, 1962

in Laocai, Vietnam 
This dissertation is approved by:

Prof.dr.ir. J. van Amerongen, promotor

Dr.ir. T.J.A. de Vries, assistant promotor 


\section{SUMMARY}

The aim of this research is to develop advanced controllers for electromechanical motion systems. In order to increase efficiency and reliability, these control systems are required to achieve high performance and robustness in the face of model uncertainty, measurement noise, and reproducible disturbances.

Proportional Integral Derivative (PID), Linear Quadratic Gaussian (LQG), Model Reference Adaptive Systems (MRAS) are typical conventional approaches where control designs involve compromises between conflicting goals. In general, for the PID control design, a compromise has to be made between performance and robust stability. In the LQG design the main issue is to trade-off attenuation of the process disturbances and the fluctuations created by measurement noise that is injected in the system due to the feedback. Direct MRAS offers a potential solution to reduce tracking errors when there are large changes in the process parameters. However, this control algorithm may fail to be robust to measurement noise. Indirect MRAS offers an effective solution to improve the control performance in the presence of parametric uncertainty and measurement noise. However, a small tracking error cannot always be obtained.

For motion control systems, Learning Feed-Forward Control (LFFC) may be used as a framework for solving the problems of reproducible disturbances. The solution is in the form of a two-degree-of-freedom controller, whose feedback and feed-forward paths independently provide the appropriate signals for disturbance rejection and model uncertainty. The use of LFFC can improve not only the disturbance rejection, but also the stability robustness of the controlled systems. Two main distinct methods, namely Neural Network (NN)-based LFFC and Model Reference Adaptive Systems (MRAS)-based LFFC, are investigated. Although both methods have been studied, little effort has been devoted to comparing or combining them. In this thesis, designs of NN-based LFFC and of MRAS-based LFFC have been developed for the high-performance robust motion control of a linear motor and a comparison of the two approaches has been evaluated. It is our first main contribution.

One of the main drawbacks of the NN-based LFFC is the requirement that the training motions are chosen carefully, such that all possibly relevant input combinations are covered. This requirement may be quite restrictive in practical applications. MRAS-based LFFC can be used to overcome such problem. By implementing both controllers on the Tripod setup, the performances of each method are compared. The experimental results show that both control algorithms reach almost the same tracking error after convergence and are superior to the PID controller. However, after convergence the MRAS-based LFFC is able to generate a much better feed-forward control and hence obtain about a 5 times smaller maxi- 
mum tracking error than the NN-based LFFC with an untrained reference motion. The reason for this is that MRAS-based LFFC can quickly generate appropriate actions for any coming input change. Moreover, compared to the NN-based LFFC, the MRAS-based LFFC is simpler to implement. The resulting control laws are simple and thus interesting for use in practical applications. Furthermore, an important difference is that the stability of the MRAS-based LFFC is analyzed by the Lyapunov theory. In other words, the stability properties of the MRAS-based LFFC are better understood.

We have shown that a combination of NN-based LFFC (to deal with nonlinearities, like cogging) and MRAS-based LFFC is superior to both systems alone. The combination performs better with respect to tracking errors and speed of learning and is simpler to realize. This is our second contribution. Both NNbased LFFC and MRAS-based LFFC can achieve good disturbance rejection, but the performance is limited by measurement noise.

In recent researches, variants of LQG have been used in combination with other algorithms to achieve better performance. Our third main contribution is the design of LQG combined with MRAS-based LFFC. This is a robust, highperformance control scheme that combines the advantages and overcomes the disadvantages of both types of techniques. In comparison to a PID controller, the LQG combined with MRAS-based LFFC has the following benefits: (1) it significantly reduces the tracking errors for any input reference signal; (2) it effectively improves the robustness with respect to changes in plant parameters and respect to measurement noise; (3) it can obtain faster transient response. 


\section{SAMENVATTING}

Het doel van dit onderzoek is het ontwikkelen van geavanceerde regelaars voor elektro-mechanische bewegingssystemen. Om de efficintie en betrouwbaarheid te vergroten dienen deze regelaars een hoog prestatie- en robuustheidsniveau te realiseren, ondanks de aanwezigheid van modelonzekerheid, meetruis en reproduceerbare verstoringen.

PID (proportioneel, integrerend, differentirend), LQG (lineair, kwadratisch, Gaussisch), of MRAS (model-referentie adapterende systemen) zijn kenmerkende conventionele benaderingen, waarbij het regelaar-ontwerp een compromis vormt tussen onderling strijdige regeldoelen. Voor een PID-ontwerp is het in het algemeen noodzakelijk om de haalbare prestatie te beperken ten gunste van een robuuste stabiliteit. In het LQG-ontwerp ligt de nadruk op het uitruilen van de onderdrukking van procesverstoringen tegen variaties die ontstaan door meetruis die het systeem binnenkomt als gevolg van het aanbrengen van terugkoppeling. Directe MRAS biedt een mogelijke oplossing voor het reduceren van volgfouten wanneer er grote veranderingen optreden in de procesparameters. Echter, dit regelalgoritme zal doorgaans geen robuustheid voor meetruis kunnen bieden. Indirecte MRAS vormt een effectieve oplossing voor het verbeteren van de regelprestatie bij aanwezigheid van parametrische onzekerheid en meetruis. Echter, een kleine volgfout wordt niet altijd verkregen.

Bij regelaars voor bewegingssystemen kan Lerende Vooruitkoppeling (LFFC) worden gebruikt als een raamwerk voor het oplossen van de problemen ten gevolge van reproduceerbare verstoringen. De oplossing heeft de vorm van een twee-graden-van-vrijheid regelaarstructuur, waarbij de terugkoppeling en de vooruitkoppeling onafhankelijk van elkaar in de signalen voorzien die geschikt zijn voor het tegengaan van verstoringen en voor modelonzekerheid. Het inzetten van LFFC kan zorgen voor een betere storingsonderdrukking, en tegelijkertijd de robuuste stabiliteit van het geregelde systeem vergroten. Twee verschillende vormen zijn onderzocht, namelijk op Neurale Netwerken (NN) gebaseerde LFFC en op MRAS gebaseerde LFFC. Beide methoden zijn eerder bestudeerd, maar er is nog weinig aandacht gegeven aan het vergelijken en het combineren ervan. In dit proefschrift zijn NN-gebaseerde en MRAS-gebaseerde LFFC ontwerpen ontwikkeld voor hoog-performante robuuste regeling van een lineaire aandrijving en is een vergelijkende evaluatie van de twee benaderingen uitgevoerd. Dit is onze eerste bijdrage.

Een van de belangrijkste nadelen van de $\mathrm{NN}$-gebaseerde LFFC is de vereiste dat de bewegingen voor het trainen zodanig zorgvuldig worden gekozen, dat alle mogelijk relevante combinaties van ingangs-variabelen worden afgedekt. In praktische situaties kan dit als restrictief worden ervaren. MRAS-gebaseerde LFFC 
viii

kan worden ingezet om dergelijke moeilijkheden te overwinnen. Door beide regelaars te implementeren op de Tripod opstelling zijn de prestaties van elk van de methodes vergeleken. De experimentele resultaten laten zien dat de beide regelalgoritmen na convergentie ongeveer dezelfde volgfout bezitten en superieur zijn aan de PID regelaar. Echter, na convergentie is de MRAS-gebaseerde LFFC in staat om veel betere voorwaartse sturing en dus een omstreeks 5 keer kleinere maximale volgfout te realiseren ten opzichte van de NN-gebaseerde LFFC voor een niet-getrainde beweging. De reden hiervan is dat MRAS-gebaseerde LFFC snel geschikte regelacties kan genereren voor elke verandering van de ingangsvariabelen. Bovendien is de MRAS-gebaseerde LFFC in vergelijking met de NNgebaseerde LFFC eenvoudiger te implementeren. De resulterende regelwetten zijn eenvoudig en dus interessant voor gebruik in realistische toepassingen. Voorts is een belangrijk verschil dat de stabiliteit van de MRAS-gebaseerde LFFC is geanalyseerd met behulp van de theorie van Lyapunov. Met andere woorden, de stabiliteitseigenschappen van de MRAS-gebaseerde LFFC zijn beter begrepen.

We hebben laten zien dat een combinatie van NN-gebaseerde LFFC (nodig voor niet-lineariteiten zoals krachtrimpel) en MRAS-gebaseerde LFFC superieur is aan beide systemen individueel. De combinatie presteert beter met betrekking tot volgfouten en snelheid van leren en is eenvoudiger te realiseren. Dit is onze tweede bijdrage. Zowel NN-gebaseerde LFFC als MRAS-gebaseerde LFFC is in staat om verstoringen te onderdrukken, maar de prestaties worden beperkt door meetruis.

In recent onderzoek zijn varianten van LQG ingezet in combinatie met andere algoritmes om te komen tot betere prestatie. Onze derde bijdrage is het ontwerp van LQG gecombineerd met MRAS-gebaseerde LFFC. Dit vormt een robuust, hoog-performant regelschema dat de voordelen samenbrengt en de nadelen oplost van beide technieken. In vergelijking met een PID-regelaar heeft de LQG gecombineerd met de MRAS-gebaseerde LFFC de volgende voordelen: (1) de volgfouten voor willekeurige gewenste ingangssignalen zijn significant verkleind; (2) de robuustheid voor veranderingen in procesparameters en voor meetruis zijn op effectieve wijze verbeterd; (3) de responsie op overgangen is versneld. 
To my parents, my sister Canh-Ty, my brothers Hung-Tam and Cuong-Cham, to my wife Linh, and to Thanh Van - Duy An 



\section{Contents}

1 Introduction 1

1.1 Advanced Controllers for Motion Systems - Why? . . . . . . . . . 1

1.2 Disturbances in electromechanical motion systems . . . . . . . 2

1.2.1 Plant reproducible disturbances . . . . . . . . . . 2

1.2.2 Measurement noise . . . . . . . . . . . . . . 4

1.2.3 Model uncertainty . . . . . . . . . . . . . . 5

1.3 Design of Control Systems _... . . . . . . . . . . 5

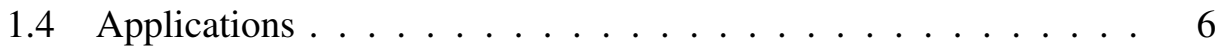

1.4.1 The MeDe5 . . . . . . . . . . . . . . . 6

1.4 .2 The Tripod . . . . . . . . . . . . . . 9

1.5 Aim and outline of the thesis . . . . . . . . . . 10

2 A review of Classical and Advanced Controllers 13

2.1 Introduction . . . . . . . . . . . . . . . 13

2.2 PID Controller . . . . . . . . . . . . . . . . . . . 14

2.3 Linear Quadratic Gaussian . . . . . . . . . . . . . . . . 17

2.3.1 Linear Quadratic Regulator . . . . . . . . . . . . . . 17

2.3.2 Linear Quadratic Estimator . . . . . . . . . . . . . . . . . 19

2.3.3 Linear Quadratic Gaussian . . . . . . . . . . . . . . . . 20

2.3.4 Discrete LQG design . . . . . . . . . . . . . . . . 22

2.4 Model Reference Adaptive Systems . . . . . . . . . . . . . . 25

2.4 .1 Direct MRAS . . . . . . . . . . . . . 26

2.4 .2 Indirect MRAS . . . . . . . . . . . . . 28

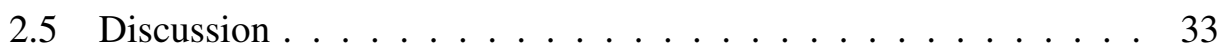

3 Neural Network based Learning Feed-Forward Control 35

3.1 Introduction . . . . . . . . . . . . . 35

3.2 Learning Control . . . . . . . . . . . . . . . . 36

3.3 Feedback-error learning . . . . . . . . . . . . . . . 37

3.3.1 Control Structure . . . . . . . . . . . . . . . . 37

3.3.2 Plant disturbance compensation . . . . . . . . . 38 
3.3.3 Multi-Layer Perceptron (MLP) neural network . . . . . . 39

3.4 Learning feed-forward control using B-spline neural network . . . 40

3.4.1 Control structure . . . . . . . . . . . . . 40

3.4.2 B-spline neural network .............. 41

3.4.3 Design of LFFC using BSNs . . . . . . . . . . . . . . 42

3.5 Input selection of the LFFC . . . . . . . . . . . . . . . . . . . . . . . . . . . . . . .

3.6 B-spline distribution . . . . . . . . . . . . . . . . . 45

3.7 Parsimonious LFFC . . . . . . . . . . . . . . . . . . . . . . . . . . . . . . 46

3.8 Phase correction for LFFC . . . . . . . . . . . . . . . . . 47

3.8.1 Un-delayed learning . . . . . . . . . . . . . . . 47

3.8.2 Delayed learning . . . . . . . . . . . . . . . . 48

3.8.3 Phase-corrected learning feed-forward control . . . . . . . . 49

3.9 Design of a parsimonious LFFC . . . . . . . . . . . . . . . . . 49

3.10 Conclusions . . . . . . . . . . . . . . . . . . 59

4 LQG combined with MRAS-based LFFC

4.1 Introduction . . . . . . . . . . . . . . . . . 61

4.2 An MRAS-based Learning Feed-Forward Controller . . . . . . . 62

4.2.1 Theoretical Background . . . . . . . . . . . . 62

4.3 Design of the proposed controller . . . . . . . . . . . . . 68

4.3.1 Design the MRAS-based LFFC . . . . . . . . . . . . . 69

4.3.2 Discrete LQG design . . . . . . . . . . . . . . . . 74

4.3.3 Solution for friction compensation . . . . . . . . . 77

4.3.4 Solution for cogging compensation . . . . . . . . . 80

4.4 comparison with the conventional PID controller . . . . . . . 82

4.5 Discussion . . . . . . . . . . . . . . . . . . 82

4.6 Conclusions ....................... 83

5 Applications $\quad 85$

5.1 Introduction . . . . . . . . . . . . . . . . 85

5.2 Implementation for the MeDe5 . . . . . . . . . . . . . 86

5.3 Implementation for the Tripod . . . . . . . . . . . . . . . 90

5.3.1 Design of a parsimonious LFFC . . . . . . . . . . . 91

5.3.2 Design of an MRAS-based LFFC . . . . . . . . . . . . 93

5.3.3 Experimental results . . . . . . . . . . . . . 94

6 Discussion 97

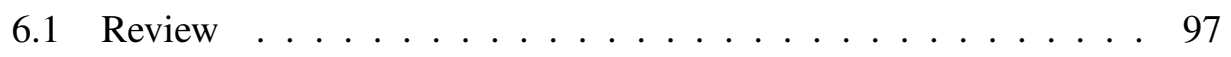

6.2 Conclusions . . . . . . . . . . . . . . . . . . 100

6.3 Recommendations for future work . . . . . . . . . . . . 101 


\section{Chapter 1}

\section{Introduction}

\subsection{Advanced Controllers for Electromechanical Motion Systems - Why?}

Motion control is concerned with manipulating power to control the movement of a mechanical system, and is widely used in packaging, printing, textile, and other industrial applications. A large amount of motion control is now performed using electric motors, so that will be our main focus. Motion control systems can be quite complicated because many different factors have to be considered in the design. The following issues must typically be considered:

- Reduction of the influence of plant disturbances

- Attenuation of the effect of measurement noise

- Variations and uncertainties in plant behavior

It is difficult to find design methods that consider all these factors, especially for the conventional control approaches where control designs involve compromises between conflicting goals. In order to design control systems to get high performance and robustness when controlling such complicated processes, advanced controllers have been introduced.

The advanced control approaches discussed in this thesis will typically focus on a few of the issues to obtain suitable solutions. The control methods will be developed gradually. We start with a simplest design approach and step by step make it more and more realistic. In a high-quality design it is often necessary to take into account all mentioned factors, such that high performance and robust stability can be achieved simultaneously. Several good properties achieved via the control structures presented in this thesis have the potential of being applied to electromechanical motion systems where linear or other controllers are unable to give the desired level of performance. 


\subsection{Reproducible disturbances, measurement noise, and plant uncertainty in electromechanical mo- tion systems}

An electromechanical motion system (see Fig 1.1) is an electrically actuated mechanical plant that requires the control of the position of the end-effector [7]. A path generator indicates a desired path for the end-effector. Electric motors used can be AC or DC, rotary or linear. Mechanical components to transform the motion of the electric motor into the end-effector (may) include: shafts, gears, belts, linkages, rotational bearings, and so on. The use of feedback in order to create a stable closed-loop system requires the use of suitable sensors to provide the necessary information on the process. These sensors are generally located at the actuator and/or the end-effector. With this structure, drawbacks for designing a

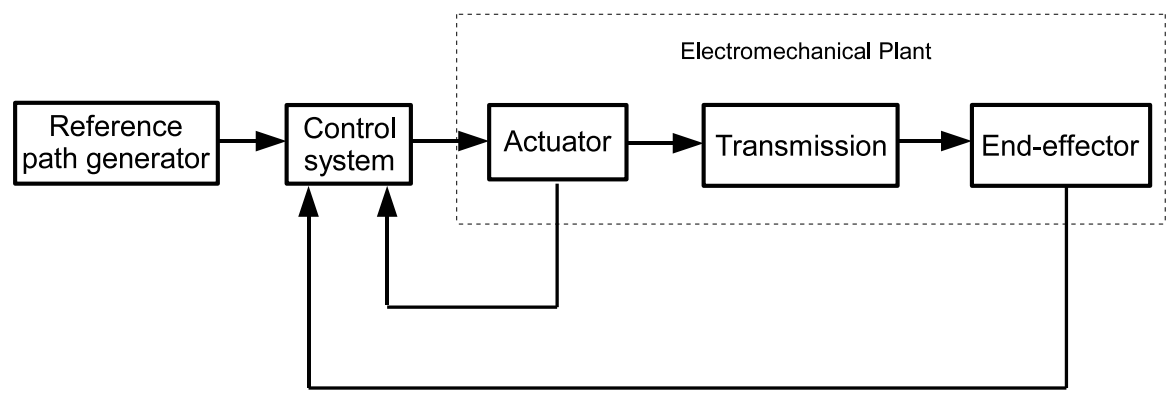

Figure 1.1: Electromechanical Motion System.

control system such as plant reproducible disturbances, model uncertainty, and measurement noise are inevitable. It is difficult to control this system with feedback alone to obtain high performance and robustness. The presence of these challenges is one of the main reasons for using advanced controllers.

\subsubsection{Plant reproducible disturbances}

A large class of motion control using an electric motor can be represented by a simple simulation model shown in Fig 1.2 [34]. The plant is always subject to disturbances. In the permanent-magnet linear motor case, the cogging force is considered as a position-dependent disturbance and the friction force is a velocitydependent disturbance. Thus, these disturbances can be viewed as a function of the state of the plant and they can have a reproducible characteristic - this means that reproducible disturbances reoccur in the same manner when a specific motion is repeated. 
The friction force is exerted in a direction that opposes movement, friction usually does negative work. As explained in [17], friction can be modeled as a velocity-dependent disturbance, which is natural to most of motion systems. Friction is highly non-linear and may cause steady-state errors, limit cycles, and poor performance. The cogging forces [1] are due to the interaction between the permanent magnets and the iron teeth of the primary section. They are especially prominent at lower speeds. They are undesirable effects that prevent smooth movement of the rotor or translator. In permanent-magnet direct drive linear motors, the cogging forces are considered to be the main disturbances. It is important for the control engineer to understand friction, and cogging phenomena and to know how to deal with them. By examining the effects of the cogging and friction disturbances it is thus possible to detect the status of the process. These reproducible disturbances may be reduced at their source. Friction force in a servo can be reduced, by using better bearings and cogging force can be minimized, by optimizing the magnet pole arc or width [1]. In this thesis we will focus on reducing effects of reproducible disturbances by means of control. In order to achieve high-precision motion control, friction and cogging must be appropriately compensated.

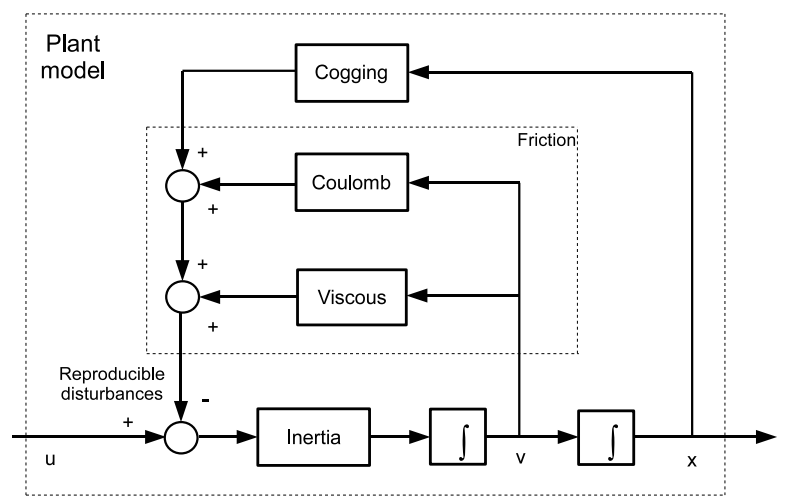

Figure 1.2: Plant model and reproducible disturbances .

Traditionally, the standard Proportional-Integral-Derivative (PID) algorithm is used in motion control to cope with the friction problem. Integral action is included in the controller to eliminate the offset. The use of feedback is often simple and effective because it is not necessary to have the detailed characteristics of the process [5]. High tracking performance can be obtained when a high gain is used in the loop. However, increasing the controller gains may make the loop unstable $[4,3]$. The friction force can also be compensated by feed-forward control but it is only suitable if the desired velocity trajectory is known in advance. The idea is simple. The friction disturbance is measured, and a control signal that attempts to counteract this disturbance is generated and applied to the process. 
In fact, friction varies depending on many factors such as normal force, position, temperature, and etc. A variation in one of these factors may change the friction characteristics in complex ways. Since friction characteristics depend on so many factors it leads to the requirement for automatic adaptation. Cogging disturbances can be well reduced by feed-forward control. Neural networks are a suitable candidate for cogging compensation. The design of a feed-forward compensator is in essence an approximation of the inverse of a dynamic system [5, 11]. In our study the compensation of state-dependent effects (such as friction and cogging) are realized by learning feed-forward control.

\subsubsection{Measurement noise}

In order to create a closed loop, it is necessary to measure the outputs of the system. This is implemented by means of sensors in the system. However, these sensors have noise associated with them, which means that the feedback signal of the system is corrupted by noise (see Fig 1.3). Next, sensor noise will be injected into the plant through the control law. Measurement noise then may be considerably amplified by feedback gains and deteriorates performance. Sensor noise in a motion control system limits the achievable bandwidth of the closed loop system. The effect of measurement noise can be attenuated, by moving a sensor to a position where there are smaller noises or by replacing a sensor with another that has less noise. In this thesis we will focus on reducing effects of

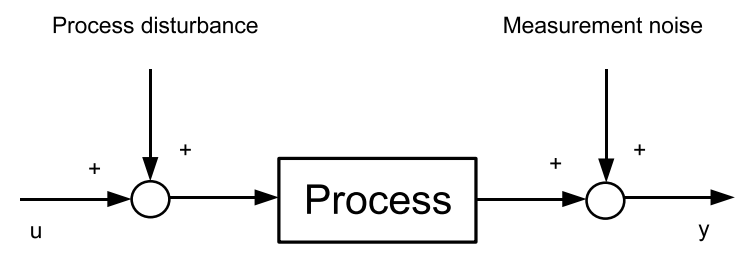

Figure 1.3: Process disturbance and measurement noise.

measurement noise by means of filtering. Kalman filters and adaptive estimators are typical examples. In fact, the control signal will often be contaminated by unwanted signals, thus filtering is necessary in order to make the process response close to the desired response. Frequently, in talking about filtering and related problems, it is implicit that the systems under control are noisy. As stated in [2], the best filter is that which, on the average, has its output closest to the correct or useful signal. As can be seen in Fig 1.3, process disturbance acts at the process input and measurement noise acts at the process output. Major issue in many control designs is a compromise between reduction of process disturbances and eliminating the fluctuations caused by the measurement noise [5]. 


\subsubsection{Model uncertainty}

In reality, motion control systems always operate with model uncertainty. Uncertainty is the absence of information, which may be described and measured. Model uncertainty may consist of parametric uncertainty and un-modeled dynamics. As explained in [20], parametric uncertainty may be caused by variable payload, poorly known masses and inertias, or by unknown and slowly time-varying friction parameters, etc. Structural uncertainty due to un-modeled dynamics may be caused by neglected friction in the drives, backlash in the gears, by neglected flexibility in joints and links, etc. In control theory, model uncertainty is considered from the points of view of physical system modeling. Un-modeled dynamics and parametric uncertainty have a negative effect on the tracking performance and can even lead to instability. If the model structure is assumed to be correct, but only accurate knowledge of plant parameters is not available, adaptive control can be applied $[4,40]$. In adaptive control, one or more control parameters and/or model parameters are adjusted on-line by an adaptation algorithm such that the closed-loop dynamics match approximately the behavior of the desired reference model despite of unknown or time varying plant parameters. Hence, to achieve asymptotic trajectory tracking, parametric uncertainty should be taken into account, under the condition that a stable closed-loop performance can be guaranteed.

\subsection{Design of Control Systems}

Most control systems are inherently nonlinear in nature. It is common to approximate them as linear mathematical models with disturbance and model uncertainty, which allows us to use the well-developed analytical design methods for linear systems. The aim of control engineering design is to obtain the configuration, specifications, and identification of the key parameters of a given system to meet an actual need [33]. Specifications are an explicit set of requirements to be satisfied by the device or product. Generally, the performance specifications given to the particular system suggest which control design method to use.

If the performance specifications are given in terms of frequency-domain performance measures and/or transient-response characteristics, then a conventional approach based on the frequency-response and/or root-locus methods may be used. With the classical control methods, the system under control is described by the input-output relationship, or transfer function. When using frequency response methods, the control designer wishes to alter the system so that the frequency response of the designed system will satisfy the specifications. When using root locus methods, the designer wishes to alter and reshape the root locus so that the 
roots of the obtained system will lie in the desired position in the s-plane. Control designs based on a conventional approach are in principle limited to linear time-invariant systems $[33,15]$.

If the performance specifications are given as performance indices in terms of state variables, then a modern control approach should be used. These specifications may include characteristics such as the energy dissipated by the system, and the required control effort. For a physical system these indices are always constrained. In modern control design, the system under control is described in state space or input-output model and control methods are mainly developed in the time domain. By using modern control approaches, the control designer is able to start from a performance index, together with constraints imposed on the system to create a stable system. The design via the context of modern control theory uses mathematical formulations of the problem and applies mathematical theory to the design problem in which the system can have multiple inputs and multiple outputs and can be time varying [33]. It enables the designer to create a designed system that is optimal with respect to the performance index.

Once performance specifications and an appropriate plant model are determined, the actual design of the control system can be established. There are many control methods for control system design. However, the preferable method is selected based on the performance specifications, the plant model, and the knowledge and experience of the designer. After a system is designed, if we can achieve the desired performance by adjusting the parameters, we will finalize the design and proceed to document the results. If it does not, we will need to change the system configuration and/or select an enhanced actuator and sensor then we will repeat the design steps until we are able to meet the required specifications. The educated trial-and-error-repetition technique is helpfully used in order to achieve the desired parameter settings.

It is commonly expected that: (1) the designed system should exhibit as small errors as possible in responding to the desired reference input, (2) the system dynamics should be relatively insensitive to changes in system parameters, and

(3) the effects of the process disturbances should be attenuated and the influence of measurement noise should be suppressed.

\subsection{Applications}

\subsubsection{The MeDe5}

For the purpose of testing the results of the controller designs for linear and nonlinear systems, The Control Engineering group of The Department of Electrical Engineering in The Faculty of Electrical Engineering, Mathematics and Computer 
Science of The University of Twente designed and implemented an experimental system named MeDe5 (The Mechatronic Demonstration Setup - 2005) (see Fig 1.4). The mechanical part of the setup is designed mimicking printer technology

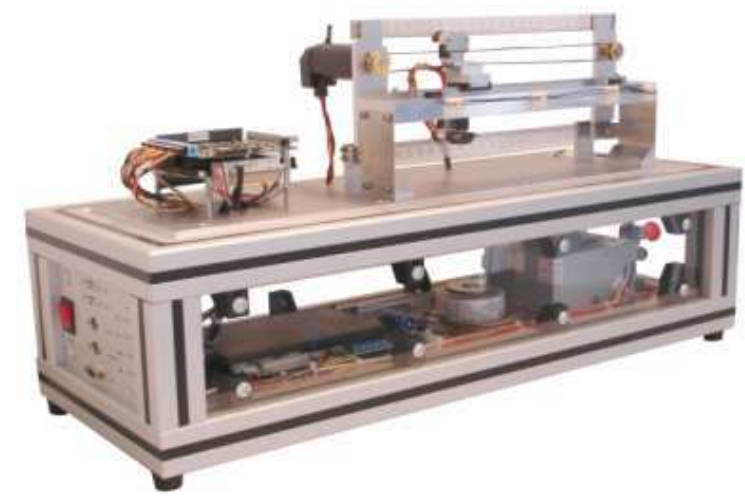

Figure 1.4: The configuration of the MeDe5 setup.

- it consists of a slider which can move back and forth over a rail. A DC motor, rail and slider are fixed on a flexible frame which causes vibration during the acceleration of the mass. For this process, a computer based control system has been implemented with software generated by 20 -sim. With 20 -sim, the behavior of dynamic systems can be modeled and simulated [41, 8]. The software tool 20-sim not only provides a modeling and simulation environment for domain independent systems. It is also provided with a C-code generator, which allows easy integration in embedded applications [14, 44].

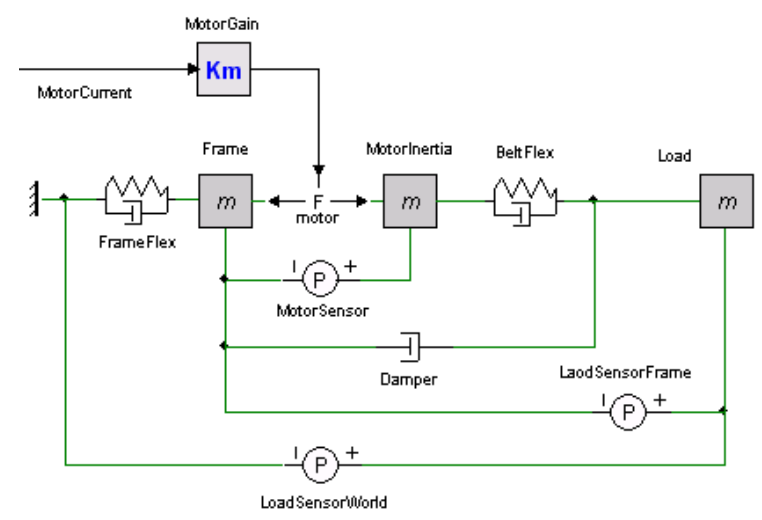

Figure 1.5: Sixth order model of the MeDe5 setup.

Fig 1.5 shows a $6^{\text {th }}$-order model of this setup. The plant parameters of this model are listed in Table (1.1). The resulting plant model is non-linear and con- 
tains high-order terms. In order to obtain a more simple plant model, linearization should be first applied and then the resulting high-order linear model should be reduced to an appropriate order. This allows us to use one or more of the welldeveloped design methods for linear systems. The model as shown in Fig 1.5

\begin{tabular}{|l|l|l|}
\hline Element & Parameter & Value \\
\hline Motor-Gain & Motor constant & $5.7 \mathrm{~N} / \mathrm{A}$ \\
\hline Frame & Mass of the frame & $0.8 \mathrm{~kg}$ \\
\hline Frame-Flex & Spring constant & $6 \mathrm{kN} / \mathrm{m}$ \\
\hline & Damping in frame & $6 \mathrm{Ns} / \mathrm{m}$ \\
\hline Motor-Inertia & Inertia of the motor & $1 \mathrm{e}-5 \mathrm{~kg}$ \\
\hline Load & Mass of the end effector (slider) & $0.3 \mathrm{~kg}$ \\
\hline Belt-Flex & Spring constant & $80 \mathrm{kN} / \mathrm{m}$ \\
\hline & Damping in belt & $1 \mathrm{Ns} / \mathrm{m}$ \\
\hline Damper & Viscous friction & $3 \mathrm{Ns} / \mathrm{m}$ \\
\hline & Coulomb friction & $0.5 \mathrm{~N}$ \\
\hline
\end{tabular}

Table 1.1: Plant parameters of the MeDe5

also contains a non-linear part. The Damper component represents a viscous and Coulomb friction. Coulomb friction always opposes relative motion and is simply modeled as

$$
F_{c}=d_{c} \cdot \tanh (1000 \cdot \dot{x})
$$

where $d_{c}$ is the Coulomb parameter of the Damper element, $\dot{x}$ is the velocity of the load. Viscous friction is proportional to the velocity. It is normally described as

$$
F_{v}=d \cdot \dot{x}
$$

where $d$ is the viscous parameter of the Damper element. The mathematical expression for the combination of viscous and Coulomb friction is

$$
F=F_{v}+F_{c}=d \cdot \dot{x}+d_{c} \cdot \tanh (1000 \cdot \dot{x})
$$

If the non-linear Coulomb friction part is disregarded, the model only contains linear components. In this case we get a linear process model. The dynamics of the $6^{t h}$-order model consist of a DC motor, a flexible frame, a flexible belt, a damper, and a load. If we assume the flexible belt to be stiff and ignore the inertia of the motor we get a linear $4^{\text {th }}$ order approximation. In the same way, if we continue to assume the flexible frame to be stiff, then we get a linear $2^{\text {nd }}$ order approximation of the process. The flexible frame causes an additional behavior, which is useful for comparing $2^{\text {nd }}$ and $4^{\text {th }}$ order control configurations. The full 
plant model is described in Appendix $A$. The MeDe5 is designed in such a way that it is convenient to test different types of controllers $[14,44]$. To predict the behavior of the controlled system, simulations are performed with both the linear reduced-order model and with the $6^{\text {th }}$-order model. Based on simulation results, frequency responses, and other tools, the design of the controller is evaluated.

\subsubsection{The Tripod}

The Tripod is a pick-and-place machine, which is manufactured by the Imotec company [16]. The Tripod is designed to test different types of advanced controllers. A picture and a schematic view of this setup are depicted in Fig 1.6 [9]. This setup consists of three linear motors, which can move up and down within their safe operating regions. A pair of rods is connected to each linear motor, and the other side of these rods is connected to the platform. Due to the constrained movement of the rods, the platform cannot rotate but only translate. The constraints on the rods make the rods form a parallelogram. The position of the platform is determined by the positions of the three linear motors. Only the position of the three linear motors is measured [9].
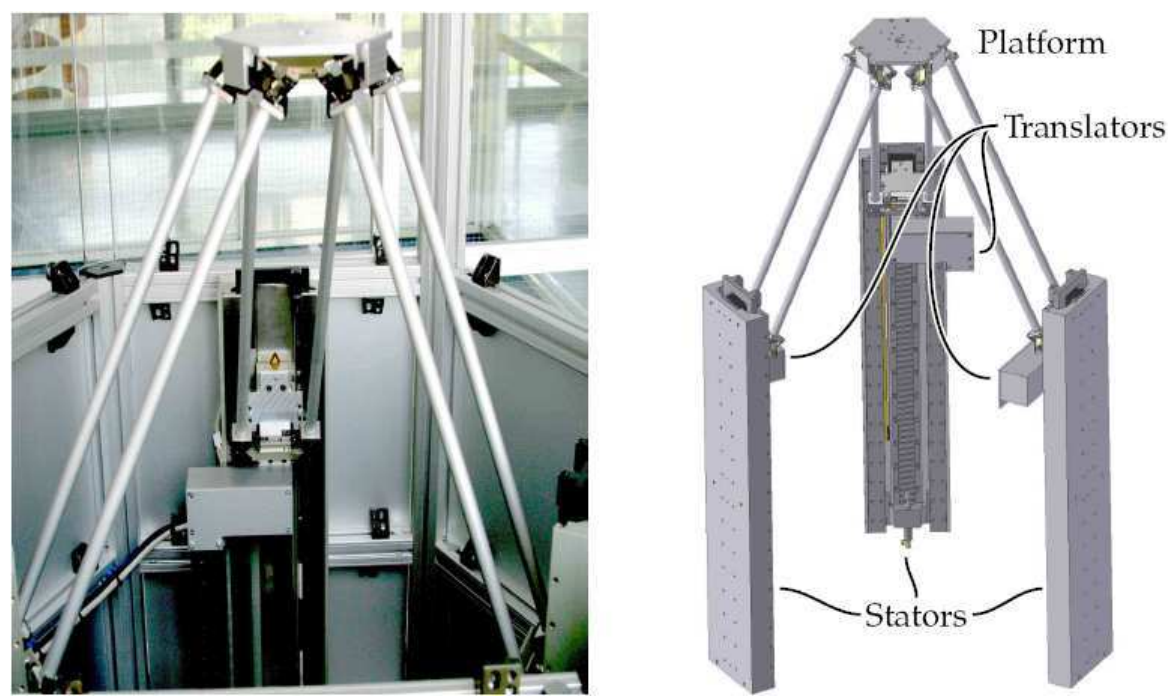

Figure 1.6: The Tripod.

In linear motors the electromagnetic force is applied directly to the payload without any mechanical transmission. This greatly reduces non-linearities and disturbances caused by backlash and additional frictional forces. Thus, linear motors are popularly used for accurate speed and position control of linear motion [1]. On the other hand, because of this direct drive construction, the cogging 


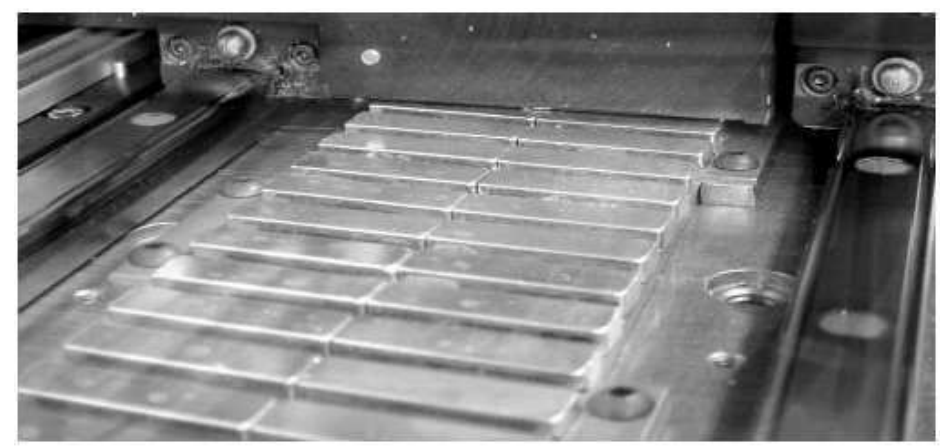

Figure 1.7: Magnets on the stator of a linear motor.

forces are not reduced by a transmission and form the main disturbance. Moreover, the friction force is a dominant disturbance. If the cogging and the friction of the linear motors are predicable, they can be compensated for by a learning feed-forward controller. This will be dealt with in Chapter 3 and Chapter 4. A plant model of a single axis of the Tripod indicated in Fig 1.2. The parameters of this model are listed in Table 1.2 [9].

\begin{tabular}{|l|l|l|}
\hline Cogging amplitude & 30 & {$[\mathrm{~N}]$} \\
\hline Cogging period & 24 & {$[\mathrm{~mm}]$} \\
\hline Coulomb friction & 12 & {$[\mathrm{~N}]$} \\
\hline Mass & $5.8-6.2$ & {$[\mathrm{~kg}]$} \\
\hline
\end{tabular}

Table 1.2: Characteristics of the linear motor

\subsection{Aim and outline of the thesis}

The aim of this research is to develop advanced controllers for electromechanical motion systems. In order to provide the necessary insight and knowledge for selecting a suitable control strategy that will be applied for a specific motion control system, this thesis is structured as follows:

\section{Chapter 2: A review of conventional and advanced controllers.}

In this chapter, more general control problems are discussed. The process model is assumed to be linear, but it may be time varying. Only brief reviews of the main ideas and results are given. This chapter starts by considering a conventional PID 
controlled system. For this type of controller, reduction of the effect of measurement noise suggests low PID gains, but attenuation of process disturbances suggests high PID gains. Both requirements cannot be achieved simultaneously. This problem can be overcome by using more advanced controllers. Next, the combination of the optimal Linear Quadratic Regulator (LQR) and Linear Quadratic Estimator (LQE) resulting in the LQG controller is discussed. This control approach enables us to trade-off between regulation performance and control effort, and to take into account process and measurement noise. However, in practice the plants are subject to parametric uncertainty and un-modeled dynamics. These uncertainties are not explicitly taken into account in the LQG design. The problem of parametric uncertainty will then be considered. This problem will be overcome by using adaptive techniques. Only two adaptive control methods, notably Model Reference Adaptive Systems (MRAS), and the Self-Tuning Regulator (STR) are considered. In the MRAS design the adaptive laws are based on the Liapunov stability theory.

\section{Chapter 3: Neural network based Leaning Feed-Forward Control}

For motion control systems, in order to get high tracking performance, the traditional feedback control should be combined with a feed-forward controller in a two Degree-of-Freedom control structure. When an accurate process model is not available, a fixed conventional feed-forward controller can be replaced by a Learning Feed-Forward controller (LFFC) [11, 12]. In this chapter, LFFC is examined in which the function approximator is implemented as a B-Spline Network (BSN).

\section{Chapter 4: LQG combined with MRAS-based LFFC}

In this chapter, the combination of LQG and MRAS-based LFFC are investigated. The control architecture is geared towards attenuating of the effects of the reproducible disturbances, measurement noise, and parameter variations and model uncertainty. The design procedure of the LQG controller was presented in Chapter 2. Hence, we focus only on the design of the MRAS-based LFFC. The setpoint generator is used as a reference model. In order to find the adaptive laws for the feed-forward parameters, the well-known Liapunov stability approach is used.

\section{Chapter 5: Applications}

This chapter deals with experiments on the Tripod setup and the MeDe5 setup. NN-based LFFC introduced in Chapter 3 and MRAS-based LFFC introduced in Chapter 4 are tested on the Tripod and the LQG combined with MRAS-based LFFC developed in Chapter 4 is tested on the MeDe5.

\section{Chapter 6: Discussion}

Firstly, we review the design procedures and experimental results of the previous chapters. Finally, conclusions are drawn. 


\section{Chapter 2}

\section{A review of Classical and Advanced Controllers}

\subsection{Introduction}

This chapter presents and evaluates various designs of controllers for electromechanical motion systems such as Proportional Integral Derivative (PID) controllers, Linear Quadratic Gaussian (LQG) controllers, and Model Reference Adaptive Systems (MRAS). The results of the design methods are simulated by 20-Sim-software and tested on the Mechatronic Demonstration Setup (MeDe5). Mainly via simulation results, from a mechatronic point of view, the positive and negative points of each method are shown and the performances are compared.

The study is to start with a conventional PID controller, which is the most common form of feedback [3]. For this type of controller, the simulation results show two main negative points. Firstly, the control signal is corrupted by measurement noise. Secondly, the tracking error depends on plant parameter variations. Therefore, simple PID controllers are not suitable for all processes. To overcome these problems, improved PID controllers or other advanced controllers have been applied. With the MeDe5 setup, the above control methods are tested. These methods are examined in both continuous and discrete time domains. The order of the linear plant model is $6[14,44]$. However, designs based on its approximation as a second and fourth order model were also implemented.

By means of a good control algorithm, acceleration and deceleration of the slider will be smooth during operation. As a result, the vibration of the flexible frame will be reduced. The higher quality control methods should show better performances. However, in fact, depending on the behavior of the plant to be controlled and the qualitative control requirements that the system has to achieve, the designer can choose the most suitable type of controllers. The best controller 
is the simplest one that is powerful enough to fulfill the system requirements.

In order to show implementation of the controllers in the presence of measurement noise and plant parameter variations, the following numerical values are fixed for most simulations: an input reference (with stroke $R=0.1[\mathrm{~m}]$, period $T=2[s]$ ), measurement noise on the slider position (with amplitude $A=5.10^{-4}$ $[\mathrm{m}]$ ), and a parameter variation in the load (with mass variation $\triangle_{m}=0.15[\mathrm{~kg}]$ ). Most of the experiments consist of three phases: $t=0-6[s]$ : homing operation, the system is controlled by a fixed PID controller; $t=6-15[s]$ : normal parameters, the system is controlled by the proposed controller; and $t=15-22[s]$ : at $t=15[s]$ a parameter variation is added to the load of the plant.

This chapter is organized as follows. In Sections 2.2 to 2.4 the results with the MeDe5 model with control methods as PID, LQG, and MRAS are shown with some comments. No detailed theoretical analyses are given in these sections. Finally, Section 2.5 summarizes the conclusions of this chapter.

\subsection{PID Controller}

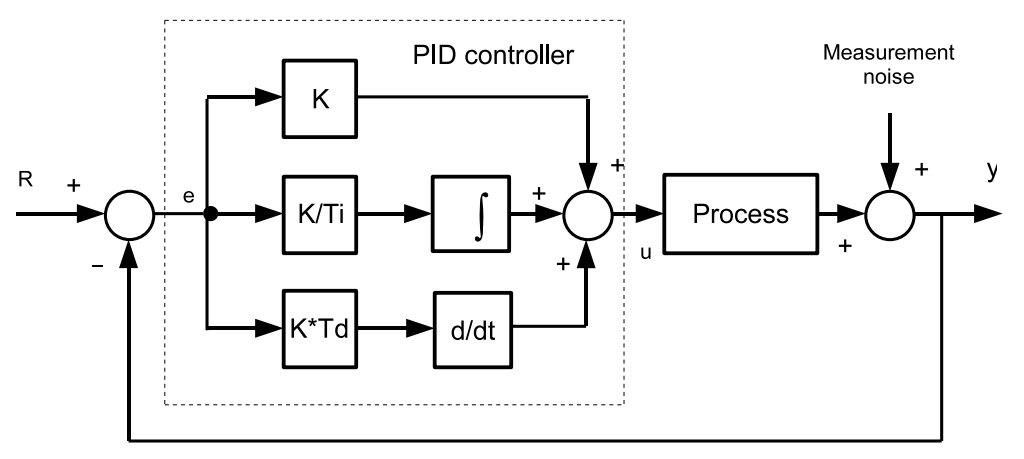

Figure 2.1: A parallel PID controlled system

A PID controller is used for most industrial control applications [4, 3]. The PID algorithm can be implemented in several ways. The parallel form is the typical form where the P, I and D components are given in parallel (see Figure 2.1). The output of the controller is given by:

$$
u(t)=K\left(e(t)+\frac{1}{T_{i}} \int_{0}^{t} e(\tau) d(\tau)+T_{d} \frac{d e(t)}{d t}\right)
$$

where, $u$ is the control signal, $e$ is the control error $(e=r-y), y$ is the measured process variable, $r$ the reference variable, $K$ is the proportional gain, $T_{i}$ the integral time, and $T_{d}$ is the derivative time. Roughly, it can be stated that all these actions have their own function [7]: 
a - The proportional action primarily provides a desired bandwidth. Larger gain results in a smaller error, and a more sensitive system. However, there will usually be a steady state error with proportional control due to Coulomb friction.

$\mathrm{b}$ - The integral action provides extra gain at low frequencies that results in suppressing low frequency disturbances. The strength of the integral action increases with decreasing $T_{i}$. The steady state error decreases when the integral action is used. Smaller $T_{i}$ implies that steady state errors are eliminated quicker, but it may make the transient response worse.

c - The derivative action mainly stabilizes loops since it adds phase lead. Larger $T_{d}$ decreases overshoot, but may slow down the transient response.

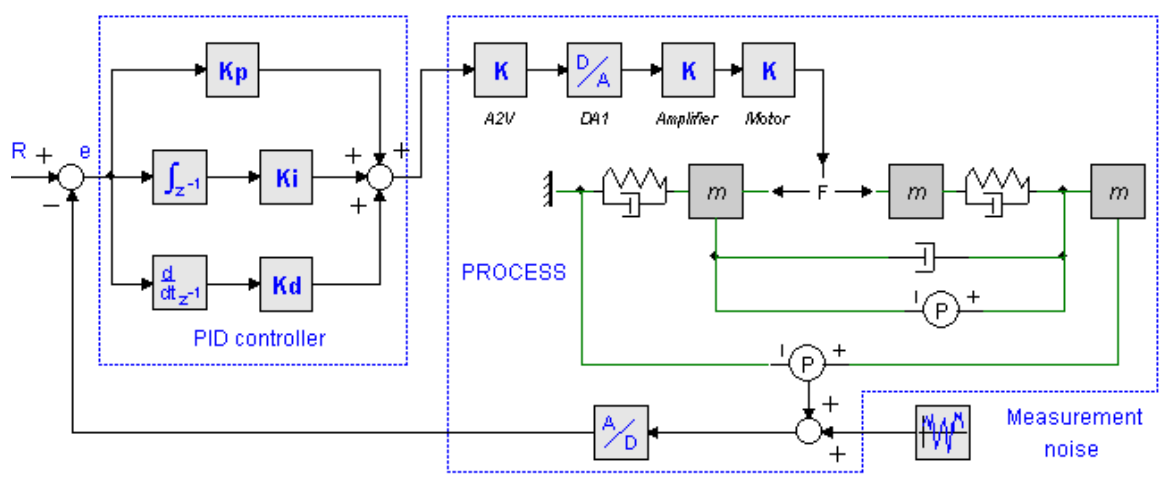

Figure 2.2: The MeDe5 with discrete PID controller

In practice some applications may require only using one or two of the controller actions. The PID structure can be simplified by setting one or two of the gains to zero, which will result in for instance a PI, PD, P or I control algorithm. In motion control, a PD algorithm can be considered as a state feedback controller for a second order system. The MeDe5 will be a system controlled by a computer, which requires a discrete control algorithm $[14,44]$. This leads to the control configuration of Fig 2.2. A more detailed procedure of the PID design can be found in [7]. Figures $2.3 \mathrm{a}$ and $2.3 \mathrm{~b}$ show the corresponding responses for the system depicted in Fig 2.2. As can be seen in Fig 2.3b, the simulation of the model can be divided in three phases $(t=0-6[s]$ : homing operation; $t=0-15[s]$ : normal parameters; $t=15-22[s]$ : a mass variation is added to the load). The simulation results in Fig 2.3a confirm that the PID controller performs poorly for this process in the presence of measurement noise. The control signal to the process (lowest line) is corrupted by measurement noise on the slider position. Another negative point of the PID controller is also shown in Fig 2.3b. When plant parameters change, the increased tracking error obtained with this structure is clearly visible. The position tracking error will increase rapidly with increasing values of the mass 

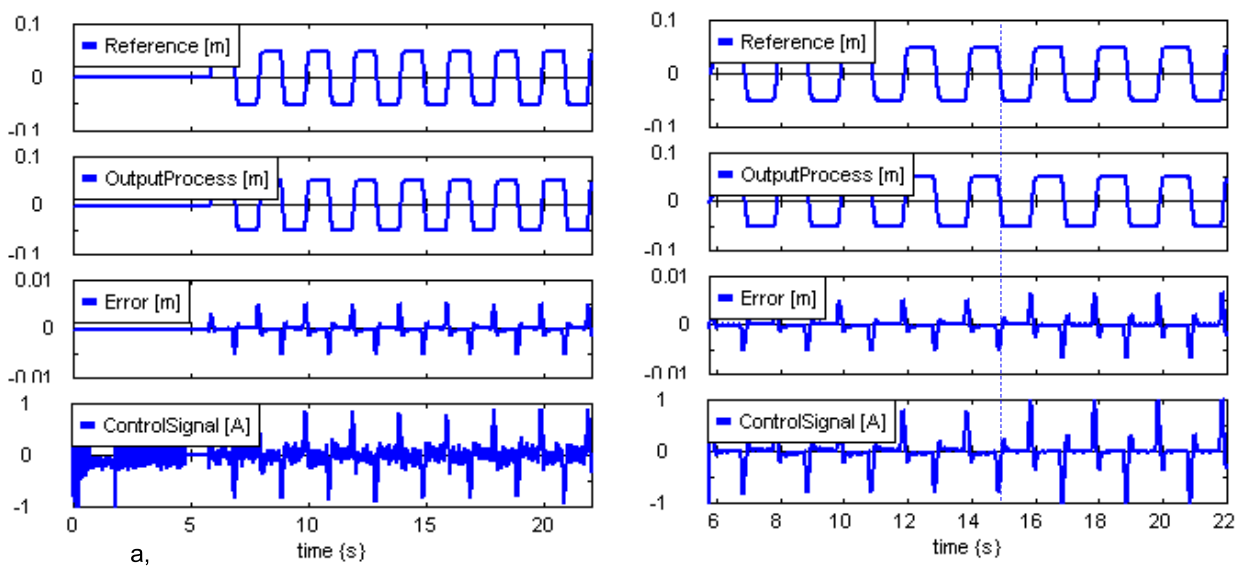

Figure 2.3: (a) Control signal is corrupted by measurement noise. (b) Position tracking error depends on plant parameter variations $(t>15[s])$

of the load ( $m=0.3[\mathrm{~kg}]$ at $t=0[\mathrm{~s}]$ and $m=0.45[\mathrm{~kg}]$ at $t=15[\mathrm{~s}])$. The simu-
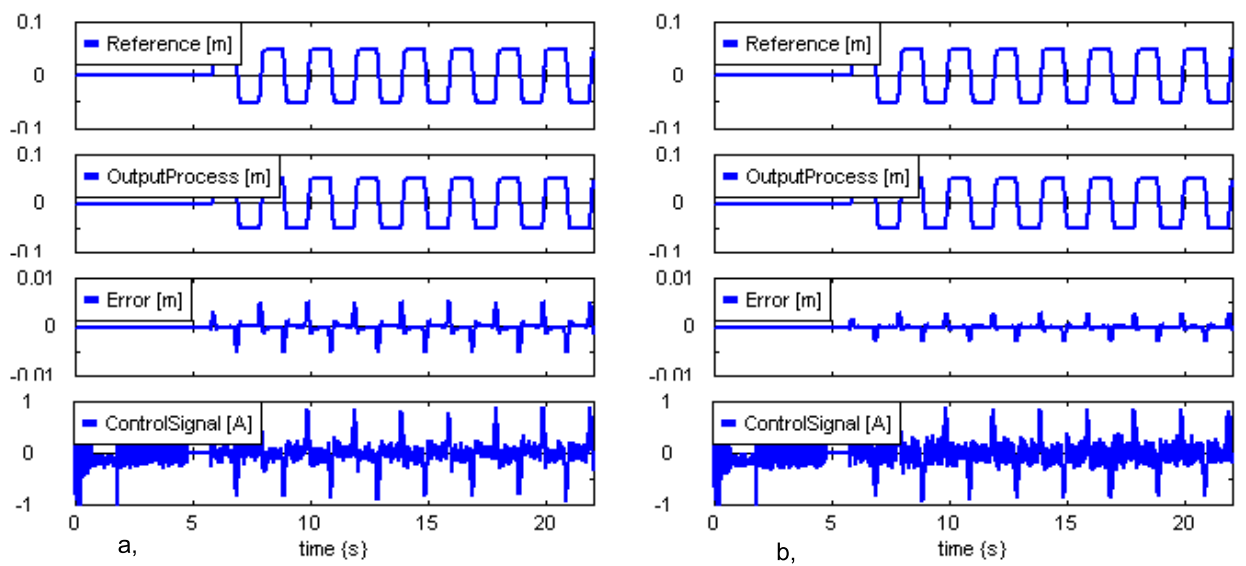

Figure 2.4: (a) Simulation results with $K_{p}=250$. (b)... with $K_{p}=500$

lation results show that the PID controlled system will be relatively insensitive to system disturbances or plant parameter variations if the proportional gain is high (see Fig 2.4). However, when the proportional gain is increased, there is a risk that the closed-loop system may become unstable due to un-modeled dynamics. Furthermore, measurement noise is amplified even more. In order to reduce the harmful effects of measurement noise to the control signal as seen in Fig 2.3a and Fig 2.4, a block diagram of a parallel PID and a Low Pass Filter is introduced in Fig 2.5. The low pass filter passes low frequencies and rejects frequencies higher 
than the cutoff frequency from its input signal. As a result, the quality of the

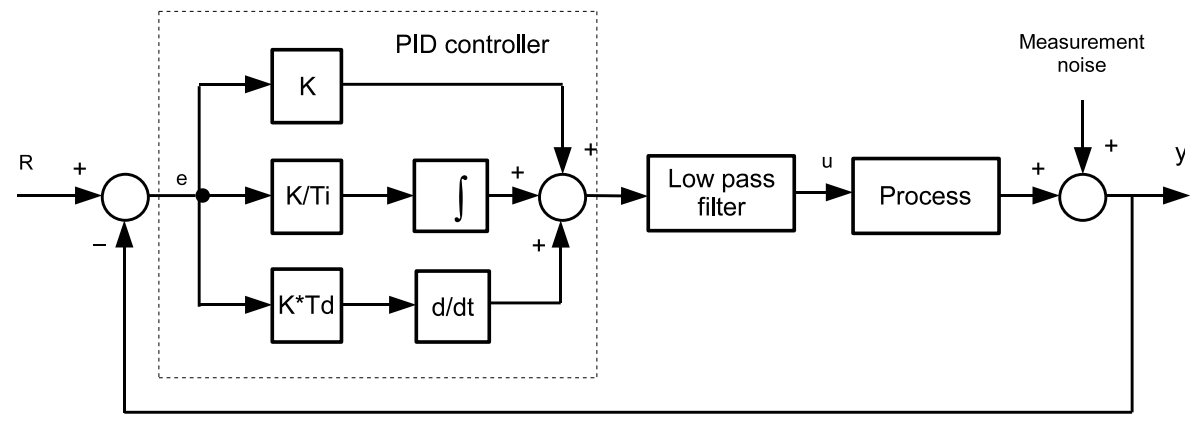

Figure 2.5: A parallel PID controlled system with a low pass filter

control signal is improved significantly in the presence of measurement noise (see Fig 2.6b). However, low pass filters have inherent disadvantages. On the one hand they may reduce the noise level, but on the other hand they produce phase shifts. They have to be tuned carefully.
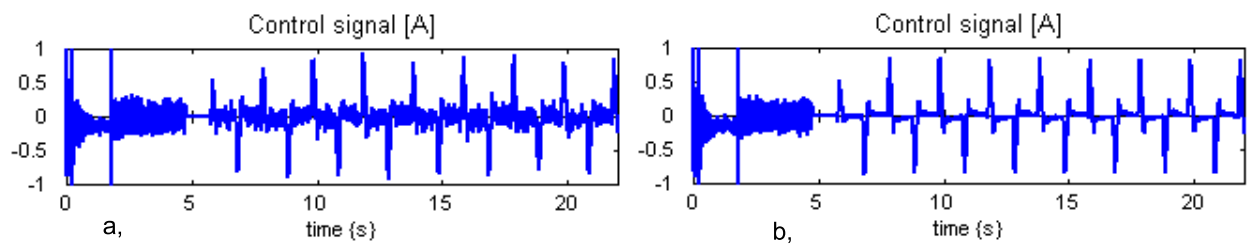

Figure 2.6: (a) Without low pass filter. (b) When a low pass filter is added

\subsection{Linear Quadratic Gaussian}

\subsubsection{Linear Quadratic Regulator}

In the theory of optimal control, the Linear Quadratic Regulator (LQR) is a method of designing state feedback control laws for linear systems that minimize a given quadratic cost function [42]. In the so called Linear Quadratic Regulator, the term "Linear" refers to the system dynamics which are described by a set of linear differential equations and the term "Quadratic" refers to the performance index which is described by a quadratic functional. The aim of the LQR algorithm is finding an appropriate state-feedback controller. The design procedure is implemented by choosing the appropriate positive semi-definite weighting matrix $Q_{R}$ and positive definite weighting matrix $R_{R}$. The advantage of the control algorithm is that it provides a robust system by guaranteeing stability margins. 


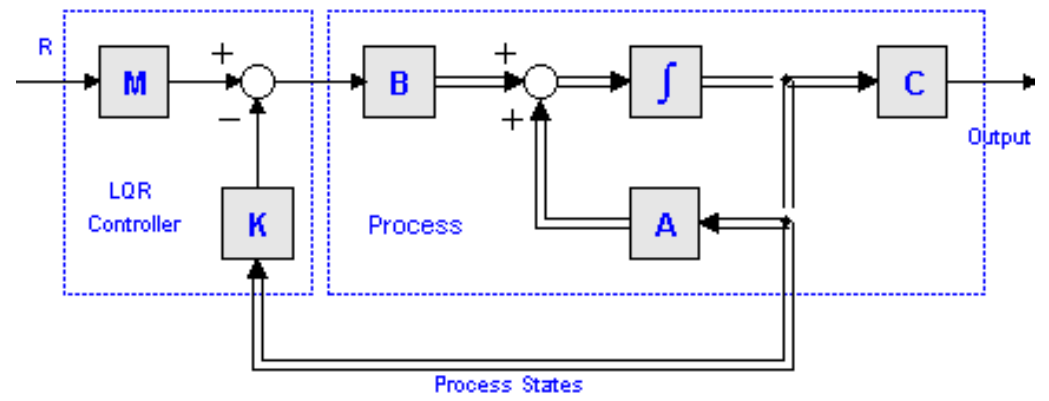

Figure 2.7: Principle of state feedback

An LQR however requires access to system state variables. A state feedback system is depicted in Fig 2.7 [42]. The internal states of the system are fed back to the controller, which converts these signals into the control signal for the process. In order to implement the deterministic LQR, it is necessary to measure all the states of the system. This can be implemented by means of sensors in the system. However, these sensors have noise associated with them, which means that the measured states of the system are not clean. That is, controller designs based on LQR theory fail to be robust to measurement noise. In addition, it may be difficult or too expensive to measure all states.

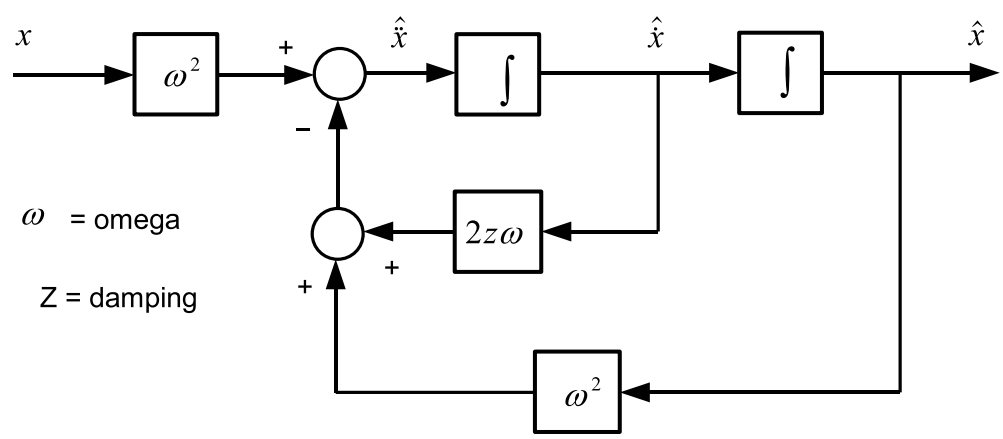

Figure 2.8: Continuous-time $2^{\text {nd }}$ order State Variable Filter

State Variable Filters (SVFs) can be used to make a complete state feedback. When the noise spectrum is principally located outside the band pass of the filter, measurement noise can be suppressed by properly choosing $\omega$ of the filter [22]. For example, in the MeD5, information about the position is measured with a lot of noise at any time instant. The SVFs remove the effects of the noise and produce a good estimate of the positions and velocities (see Fig 2.9). However, SVFs cause phase lags (see Fig 2.10). The phase lags can be reduced by means 


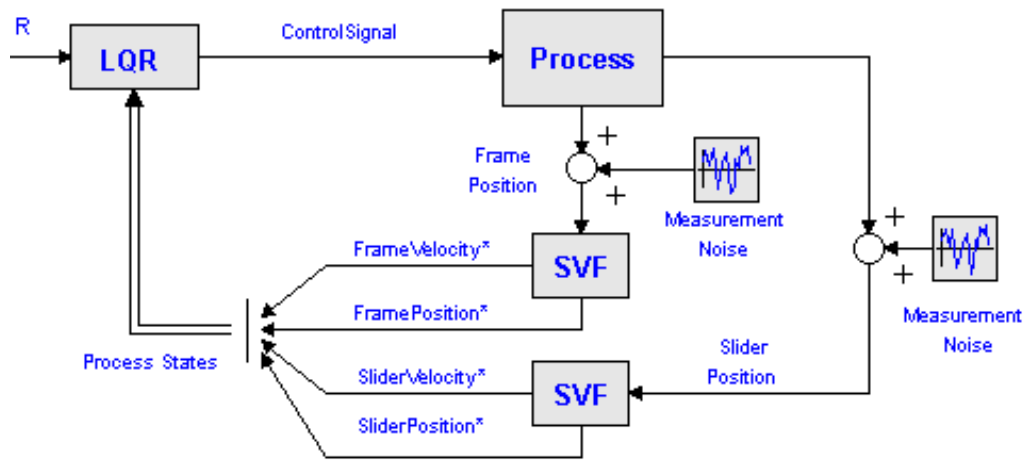

Figure 2.9: Clean state feedback of the process is obtained by using State Variable Filters

of increasing the omega of the SVF. In practice, the choice of the omega is a compromise between the phase lag and the sensitivity for noise [22].

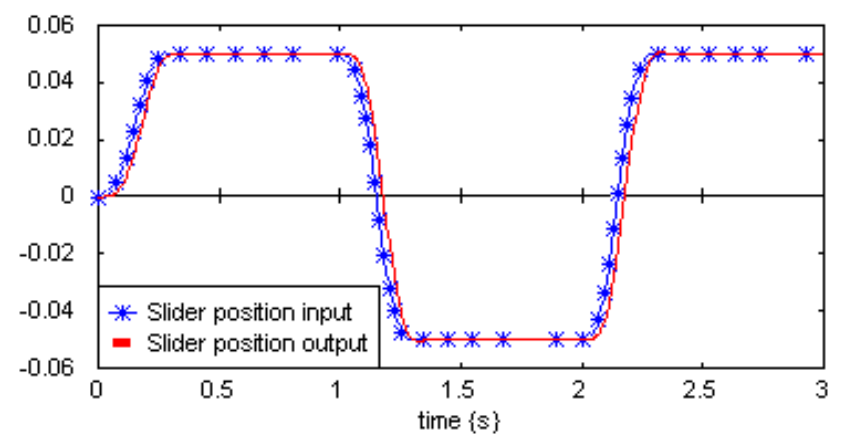

Figure 2.10: The phase lag between the input and output signal of an SVF with an omega of $50(\mathrm{rad} / \mathrm{sec})$

\subsubsection{Linear Quadratic Estimator}

Another way to estimate the internal state of the system is by using a Linear Quadratic Estimator (LQE) (see Fig 2.11). In control theory, the LQE is most commonly referred to as a Kalman filter or an Observer [42]. The Kalman filter is a recursive estimator. This implies that to compute the estimate for the current state, the estimated state from the previous time step and the current measurement are required. The Kalman filter is implemented with two distinct phases:

$\mathrm{i}$ - The prediction phase, the estimate from the previous step is used to create an estimate of the current state. 
ii - The update phase uses measurement information from the current step to refine this prediction to arrive at a new estimate.

A Kalman filter is based on a mathematical model of a process. It is driven by the control signals to the process and the measured signals. When we use Kalman filters or observers disturbances at the input of the process are mostly referred to as "system noise" as in Fig 2.11.

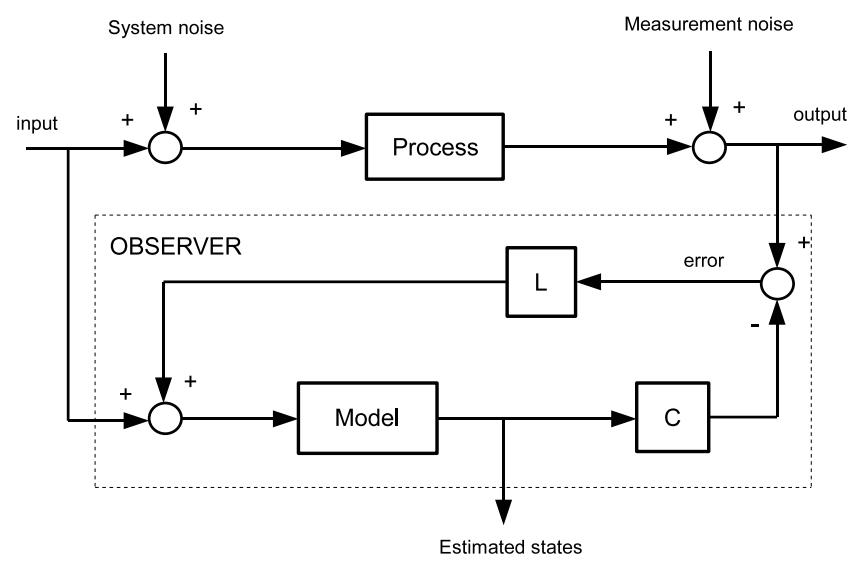

Figure 2.11: Principle of an observer

Its output is an estimate of the states of the system including the signals that cannot be measured directly. The Kalman filter provides an optimal estimate of the states of the system in the presence of measurement noise and system noise. In order to obtain optimality the following conditions must be satisfied [42]:

i - Structure and parameters of process and model must be identical.

ii - Measurement and system noise have average zero and known variance.

The LQE design determines the optimal steady-state filter gain $L$ based on linear parameters of the process, the system noise covariance $Q_{E}$ and the measurement noise covariance $R_{E}$. The states of the model will follow the states of the plant, depending on the choice of $Q_{E}$ and $R_{E}$.

\subsubsection{Linear Quadratic Gaussian}

Linear Quadratic Gaussian (LQG) is simply the combination of a Linear Quadratic Regulator (LQR) and a Linear Quadratic Estimator (LQE) [42]. This means that LQG is a method of designing state feedback control laws for linear systems with additive Gaussian noise that minimizes a given quadratic cost function. The control configuration is shown in Fig 2.12. The design of the LQR and LQE can be carried out separately. LQG enables us to optimize the system performance and 


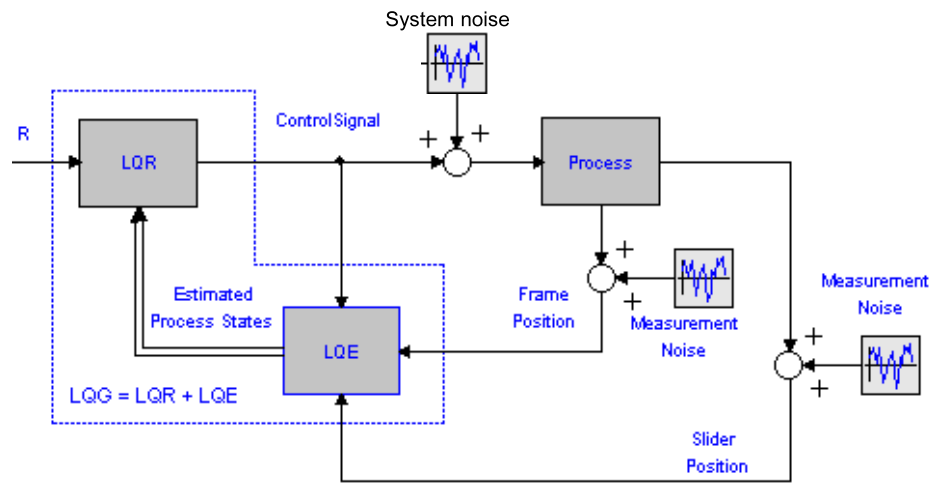

Figure 2.12: LQG explanation

to reduce measurement noise. The LQE yields the estimated states of the process. The LQR calculates the optimal gain vector and then calculates the control signal. However, in state feedback controller designs reduction of the tracking error

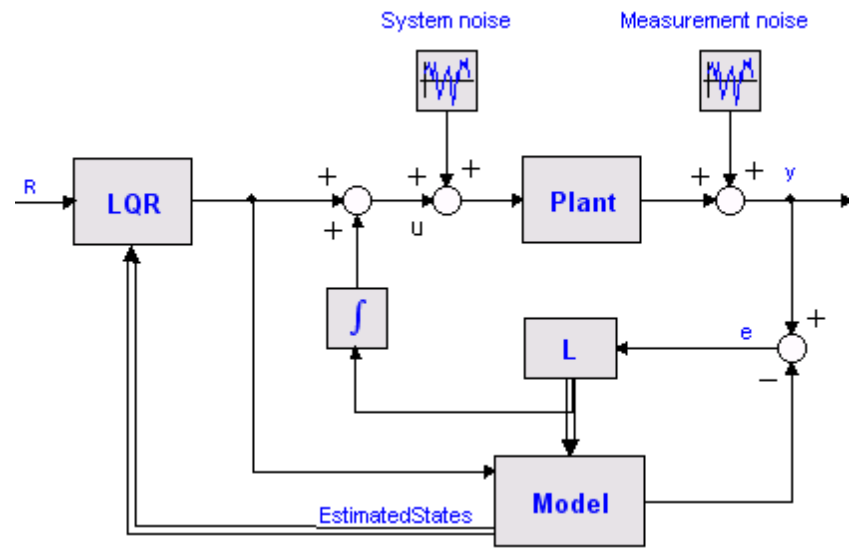

Figure 2.13: Addition of integrator to LQG

is not automatically realized. In motion control systems, Coulomb friction is the major non-linearity, which causes a static error. This problem can be solved, by introducing an additional integral action to the LQG control structure [42]. The difference between process and model is integrated, instead of the error between reference and process output (in the PID controller). Adding the integral term to the LQG control structure leads to the system indicated in Figure 2.13. 


\subsubsection{Discrete LQG design}

We consider the LQG design based on the $2^{\text {nd }}$ order approximation of the $6^{\text {th }}$ order mathematical model. A more detailed exposition of the LQG design can be found in [42]. The design procedure in here is also based on this book.

\section{Discrete LQR}

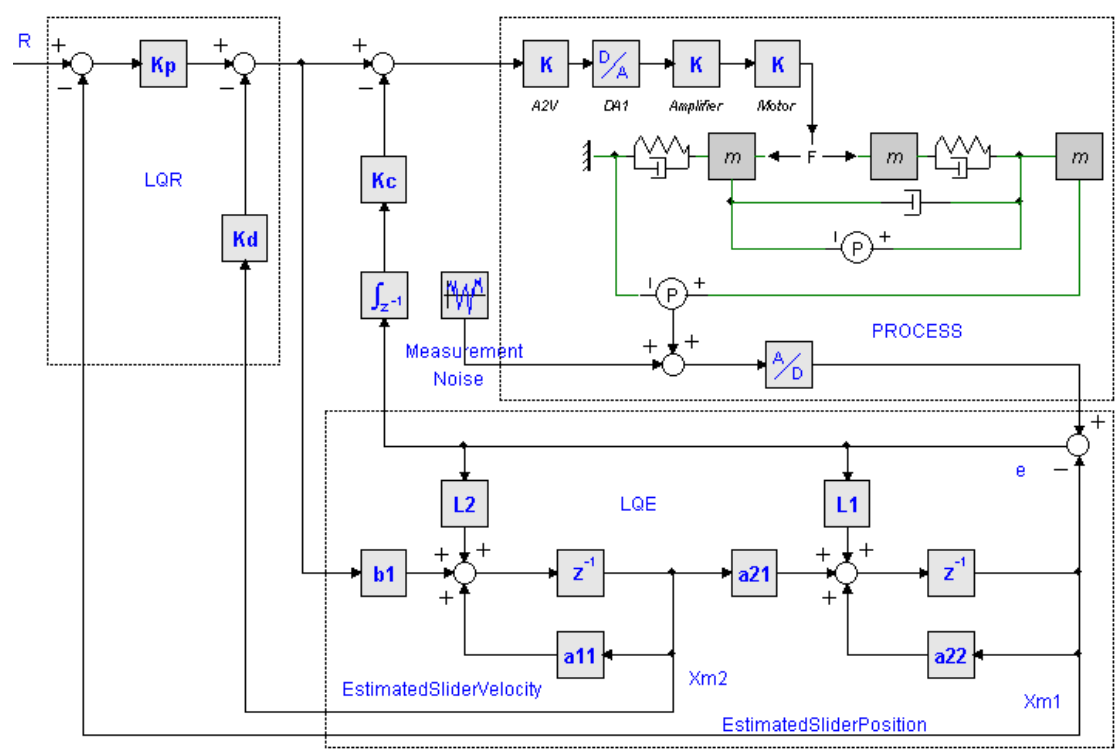

Figure 2.14: The MeDe5 with $2^{\text {nd }}$ order discrete LQG controller

We consider a discrete-time linear plant described by

$$
\left\{\begin{array}{l}
x_{k+1}=A x_{k}+B u_{k} \\
y_{k}=C x_{k}+D u_{k} .
\end{array}\right.
$$

with a performance index defined as

$$
J=\Sigma_{k=0}^{\infty}\left(e_{k}^{T} Q_{R} e_{k}+u_{k}^{T} R_{R} u_{k}\right)
$$

In equations (2.2) and (2.3) $A, B, C$ and $D$ are discrete state matrices of the plant to be controlled, $x$ denotes the state of the plant, $e$ is the tracking error, $u$ is the control signal, $Q_{R}$ and $R_{R}$ are matrices in the optimization criterion $\left(Q_{R}\right.$ is positive semi-definite weighting matrix and $R_{R}$ is positive definite weighting 
matrix). The optimal state feedback controller will be achieved by choosing a feedback vector

$$
K_{L Q R}=\left(B^{T} P B+R_{R}\right)^{-1} B^{T} P A,
$$

in which $P$ is the solution of the reduced matrix Riccati equation

$$
A^{T} P A-P-A^{T} P B\left(B^{T} P B+R_{R}\right)^{-1} B^{T} P A+Q_{R}+P=0,
$$

The output of the state feedback controller is

$$
u=-K_{L Q R} x_{m}
$$

where

$$
x_{m}=\left[\begin{array}{ll}
x_{m 2} & x_{m 1}
\end{array}\right]^{T},
$$

$x_{m 2}$ and $x_{m 1}$ are defined in Fig 2.14. The following parameters from the MeDe5 (see Appendix A) are used in the simulations:

$$
\begin{gathered}
A=\left[\begin{array}{ll}
a_{11} & a_{12} \\
a_{21} & a_{22}
\end{array}\right]=\left[\begin{array}{ll}
0.990 & 0.000 \\
0.001 & 1.000
\end{array}\right], B=\left[\begin{array}{l}
b_{1} \\
b_{2}
\end{array}\right]=\left[\begin{array}{l}
0.019 \\
0.000
\end{array}\right], \\
Q_{R}=\left[\begin{array}{cc}
0.000 & 0.000 \\
0.000 & 1000
\end{array}\right], R_{R}=0.0001 .
\end{gathered}
$$

These values result in the following stationary feedback controller gains

$$
K_{L Q R}=\left[\begin{array}{ll}
K_{d} & K_{p}
\end{array}\right]=\left[\begin{array}{ll}
18.10 & 2657.90
\end{array}\right] .
$$

\section{Discrete LQE}

The feedback matrix $L$ yielding optimal estimation of the process states is computed as

$$
L=P C^{T}\left(C P C^{T}+R_{E} I\right)^{-1},
$$

where $P$ is the solution of the following matrix Riccati equation

$$
\operatorname{next}(P)=A(I-L C) P A^{T}+Q_{E},
$$

in which $A$ and $C$ are discrete state matrices of the plant to be controlled, $Q_{E}$ is the system noise covariance, and $R_{E}$ the sensor noise covariance. The following settings were used

$$
A=\left[\begin{array}{ll}
0.990 & 0.000 \\
0.001 & 1.000
\end{array}\right], C=\left[\begin{array}{ll}
0 & 1
\end{array}\right]
$$




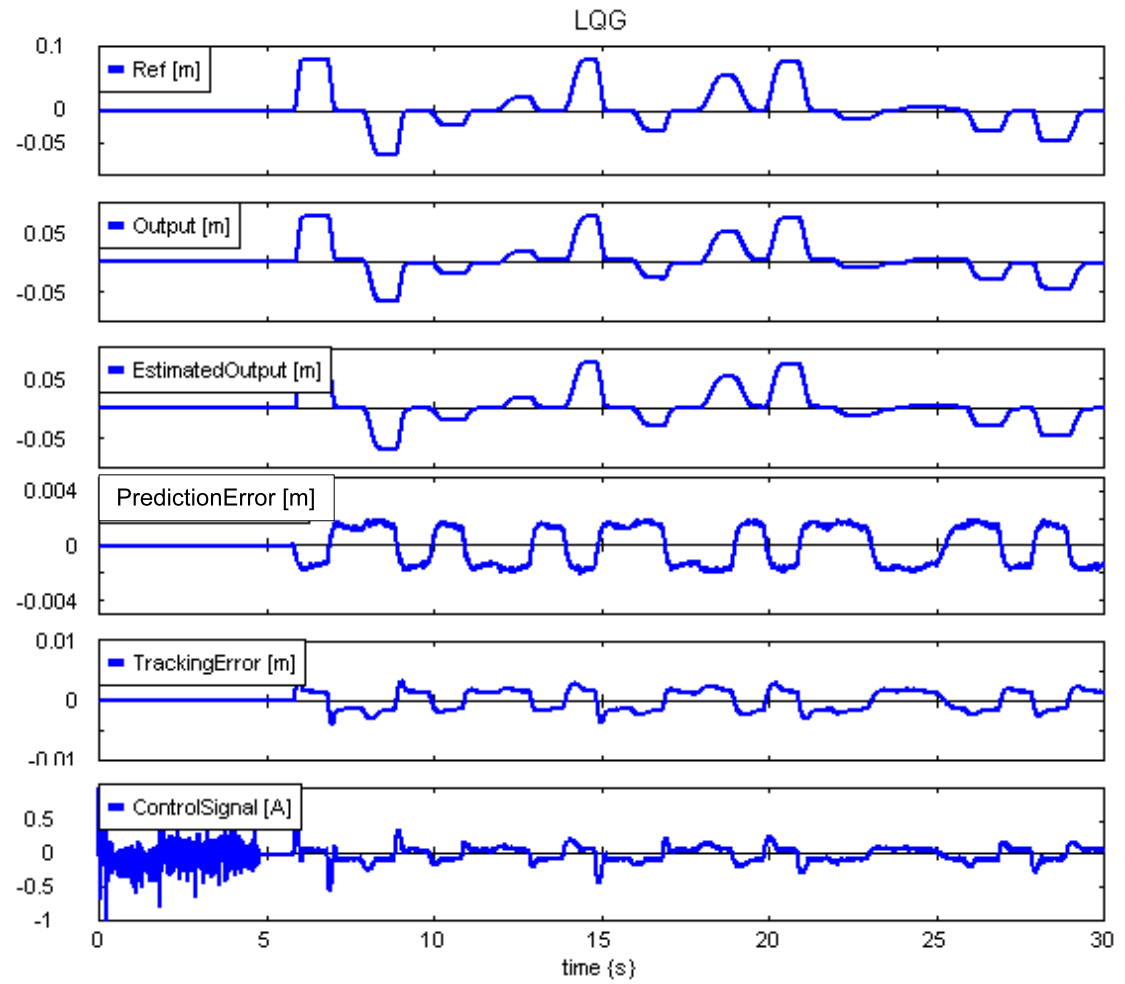

Figure 2.15: Control signal is insensitive for measurement noise

$$
Q_{E}=\left[\begin{array}{cc}
10 & 0.0 \\
0.0 & 10
\end{array}\right], R_{E}=1000 .
$$

The settings result in the following stationary gains

$$
L_{L Q E}=\left[\begin{array}{l}
L_{1} \\
L_{2}
\end{array}\right]=\left[\begin{array}{l}
0.0043 \\
0.0951
\end{array}\right]
$$

The results lead to the control structure shown in Fig 2.14. With the LQG regulator, noise on the measurements of the process has almost no influence on the system. This is illustrated in Figure 2.15; the real position state (second line) and the position state error (fourth line) are corrupted by measurement noise, whereas, the estimated position state (third line) and the control signal (lowest line) are almost clean. The integral action is used to compensate the effect of the process disturbances (see Figure 2.14). The gain of the integrator can be tuned manually, or it can be included in the solution of the Riccati equation [22]. By comparing two simulation results as indicated in Fig 2.16a and Fig 2.16b, it is observed that when the integral action is used with $K_{C}=2500$ the tracking error is decreased. 

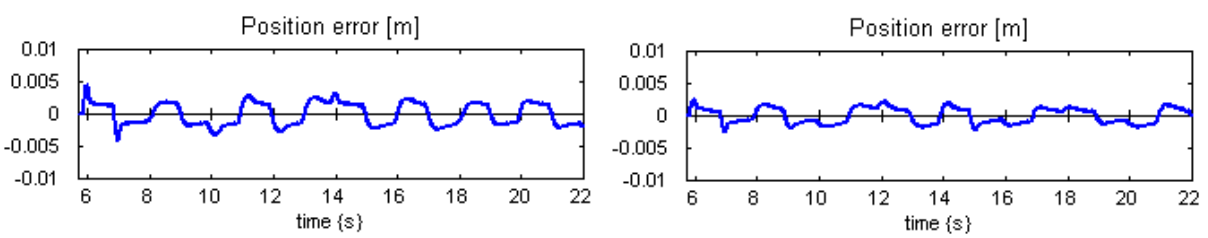

Figure 2.16: (a) Without integral action. (b) With integral action

\subsection{Model Reference Adaptive Systems}

Model reference adaptive control systems (MRAS) are one of the main approaches to adaptive control. The desired performance is expressed in terms of a reference model (a model that describes the desired input-output properties of the closed loop system). When the behavior of the controlled process differs from the "ideal

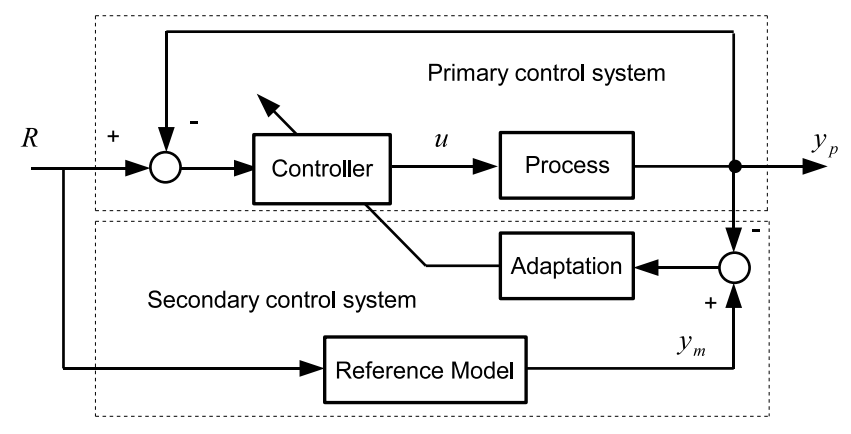

Figure 2.17: Direct MRAS - Parameter adaptive system

behavior", which is determined by the reference model, the process is modified, either by adjusting the parameters of a controller (see Fig 2.17), or by generating an additional input signal for the process (see Fig 2.18) based on the error between the reference model output and the system output [40,3]. The aim is to let parameters converge to ideal values that result in a plant response that tracks the response of the reference model. As can be seen from Fig 2.17 and Fig 2.18, there are two loops, namely an inner (primary) loop, which provides the ordinary feedback control, and an outer (secondary) loop, which adjusts the parameters in the inner loop. The inner loop is assumed to be faster than the outer loop [40].

Model reference adaptive control systems can be classified into two main classes [40, 3, 22]: The first is indirect or explicit adaptive control, in which online estimates of the plant parameters are used for control law adjustment. The second is direct, or implicit adaptive control, in which no effort is made to identify the plant parameters, that is, the control law is directly adjusted to minimize 


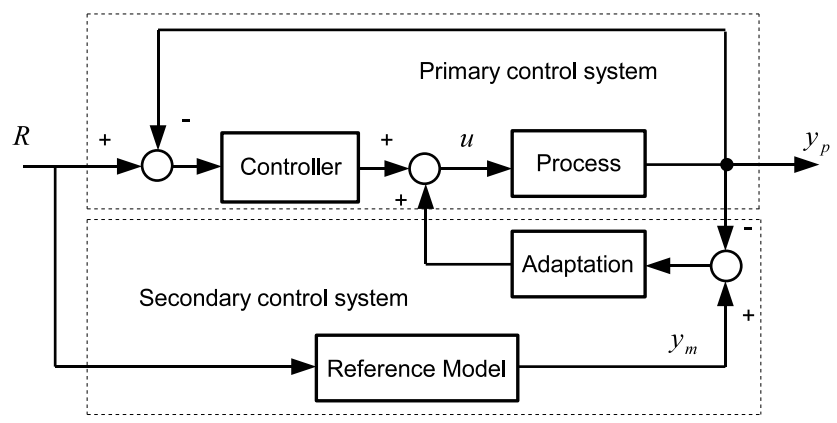

Figure 2.18: Direct MRAS - signal adaptive system

the errors between the reference outputs and the process outputs. A more detailed procedure of the MRAS design can be found in [22, 40].

The control configuration of direct MRAS is illustrated in Figure 2.17 and also in Figure 2.18. In this case the process must follow the response of the reference model. The structure depicted in Fig 2.17 can be used as an adaptive PID controlled system. The controller parameters are adapted directly by the tracking error between the plant output and the reference model output to form the control signal.

\subsubsection{Direct MRAS}

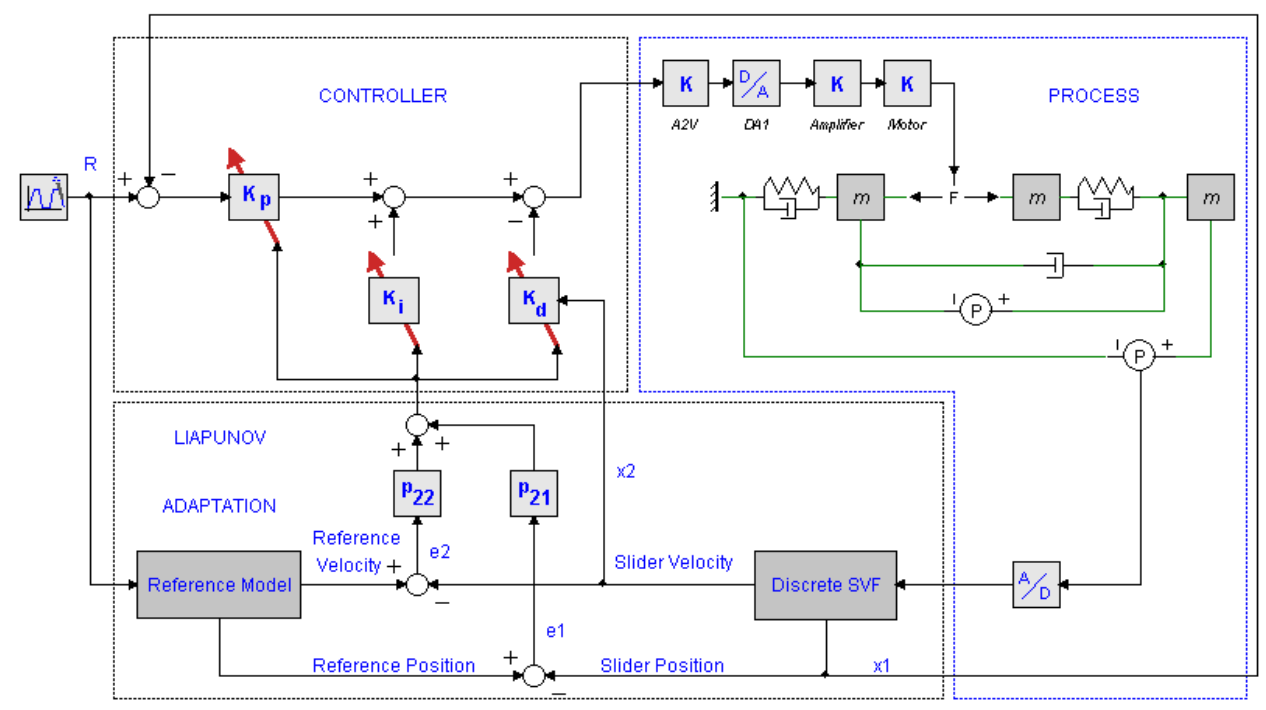

Figure 2.19: Control configuration of the adaptive PID controlled $6^{\text {th }}$ order process 
For the system shown in Fig 2.19, a state variable filter is placed after the process output in order to obtain filtered values of the measured position and velocity of the load with respect to the fixed world. The states of the reference model are compared with the filtered states of the process. The adaptive laws used in this controller are based on the Liapunov stability theory [40]. In discrete time the adjustable parameters of the controller are given by:

$$
\begin{gathered}
K_{p}=\alpha_{p} \sum\left[\left(p_{21} e_{1}+p_{22} e_{2}\right)\left(R-x_{1}\right)\right] \cdot T_{s}+K_{p}(0), \\
K_{d}=\alpha_{d} \sum\left[\left(p_{21} e_{1}+p_{22} e_{2}\right) x_{2}\right] \cdot T_{s}+K_{d}(0), \\
K_{i}=\alpha_{i} \sum\left[\left(p_{21} e_{1}+p_{22} e_{2}\right)\right] \cdot T_{s}+K_{i}(0),
\end{gathered}
$$

where $\alpha_{p}, \alpha_{d}$ and $\alpha_{i}$ are the speed of adaptation, $e_{1}, e_{2}, R, x_{1}$, and $x_{2}$ are defined in Fig 2.19, $T_{s}$ is the sampling interval, $p_{21}$ and $p_{22}$ are elements of the $P$ matrix, obtained from the solution of the Liapunov equation:

$$
\operatorname{next}(P)=\left(A_{m}^{T} P+P A_{m}+Q\right) \cdot T_{s}+P .
$$

In this equation $Q$ is a positive definite matrix; matrix $A_{m}$ belongs to the desired

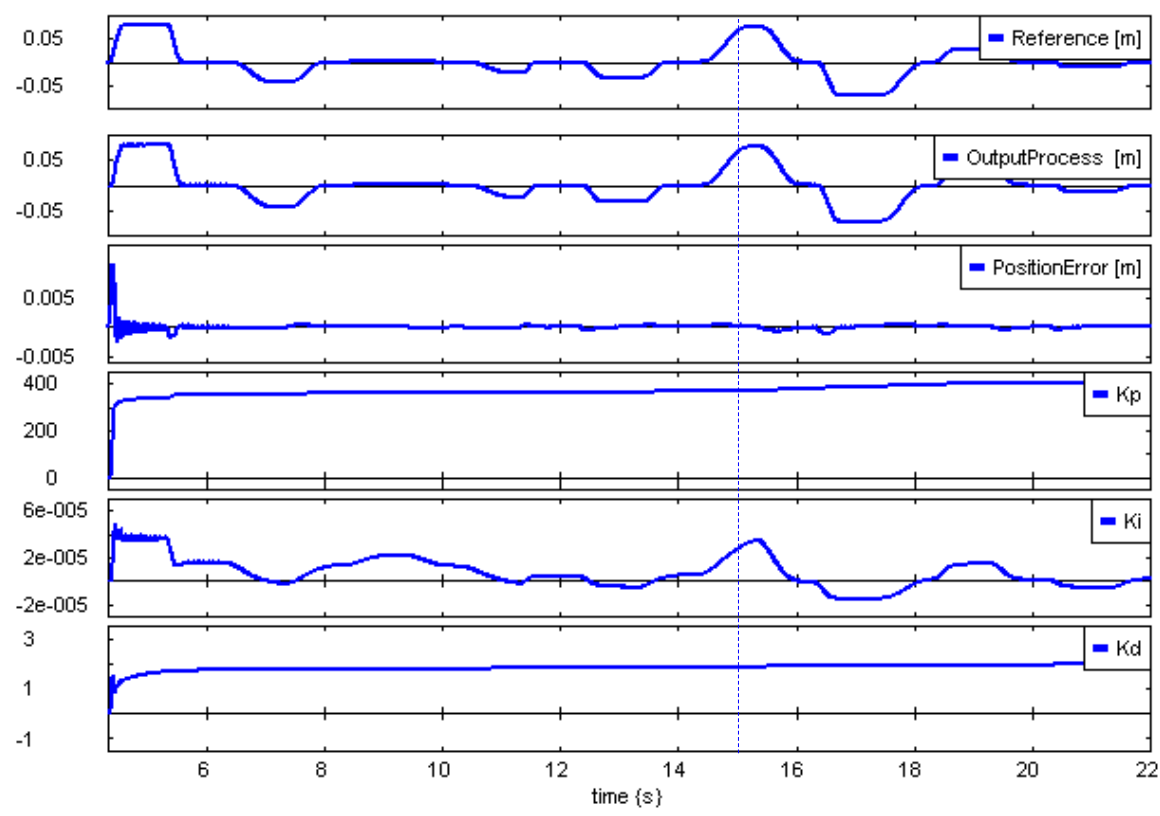

Figure 2.20: Simulation results (a load mass variation is added at $t=15[s]$ ) 
reference model. The following settings were used

$$
A_{m}=\left[\begin{array}{cc}
0.00 & 1.00 \\
-100 & -20.0
\end{array}\right], Q=\left[\begin{array}{cc}
100 & 0 \\
0 & 100
\end{array}\right], T_{s}=0.001
$$

The settings result in the steady state values

$$
p_{21}=0.3785, p_{22}=0.8359 \text {. }
$$

The following speeds of adaptation are chosen

$$
\alpha_{p}=5000, \alpha_{d}=50, \alpha_{i}=25
$$

The control configuration as used for the adaptive controlled process given in Fig 2.19 is based on the structure indicated in Figure 2.17. As can be seen in Fig $2.20 K_{p}$ and $K_{d}$ reach stationary values while $K_{i}$ remains varying to maintain a small position tracking error. In the beginning the maximum tracking error is large. However, when the adaptive gains $K_{p}$ and $K_{d}$ reach stationary values, it will decrease quickly to a small value.

By comparing two simulation results as indicated in Fig 2.3b and Fig (2.20 third line), it can be seen that in the direct MRAS case, after a load mass variation at $t=15[s]$, the position tracking error quickly converges to a small value. The conventional PID controller cannot do that. In other words, with respect to representative load variations, the direct MRAS algorithm is more robust than the PID algorithm. However, negative point of the direct MRAS is shown in Fig 2.21. The control signal is corrupted by measurement noise.

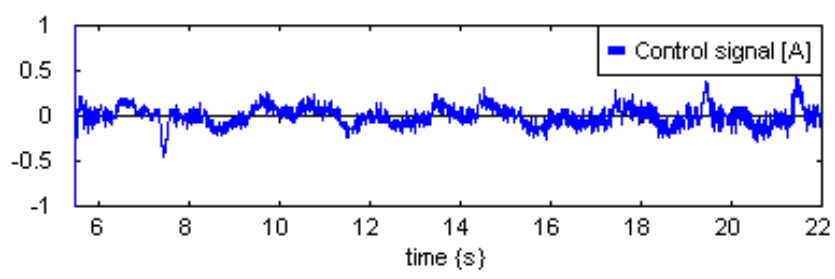

Figure 2.21: Control signal is corrupted by measurement noise

The adaptive gains, which determine the speed of adaptation, can in principle be chosen freely [40]. However, un-modeled system dynamics limit these values in practice.

\subsubsection{Indirect MRAS}

Fig 2.22 shows the block diagram of the indirect MRAS, which combines an adaptive observer and an adaptive Linear Quadratic Regulator (LQR). The function of 
this adaptive observer and the function of the LQE block, which is indicated in Fig 2.12, are almost the same. The control scheme consists of two phases at each time step. The first phase consists of identifying the process dynamics by adjusting the parameters of the model. In the second phase, the adaptive LQR design is implemented, not from a fixed mathematical model of the process, but from the identified model.

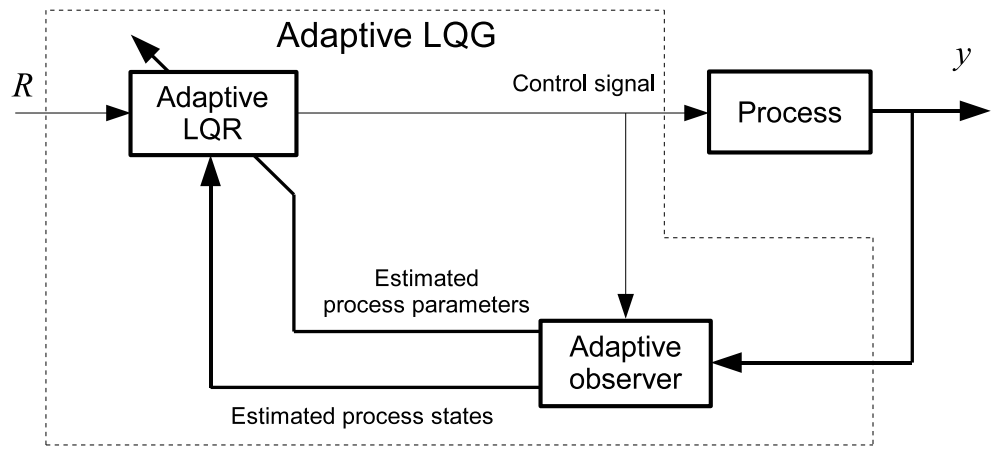

Figure 2.22: Adaptive LQG = Adaptive LQR + Adaptive Observer

\section{Adaptive observer}

The reference model, in this case referred to as the 'adjustable model', will follow the response of the process [40]. In the following discussions the terms "adjustable model" and "adaptive observer" are used interchangeably. The goal in process identification is to obtain a satisfactory model of a real process by observing the process input-output behavior. Identification of a dynamic process contains four basic steps [22]. The first step is structural identification, which allows us to characterize the structure of the mathematical model of the process to be identified. This can be done from the phenomenological analysis of the process. Next, we determine the inputs and outputs. Third step is parameter identification. This step allows us to determine the parameters of the mathematical model of the process. Finally, the identified model is validated. When the parameters of the identified model and the process are supposed to be "identical", the model states can be considered as estimates of the process states. When the states of the process are corrupted with noise, the structure of the adaptive observer can be used to get filtered estimates of the process states. When the input signal itself is not very noisy, the model states will also be almost free of noise (see Fig 2.24). It is important to notice that in this case the filtering is realized with minimum phase lag [40], like the LQE. However, this observer is also able to deal with unknown or time-varying parameters. 


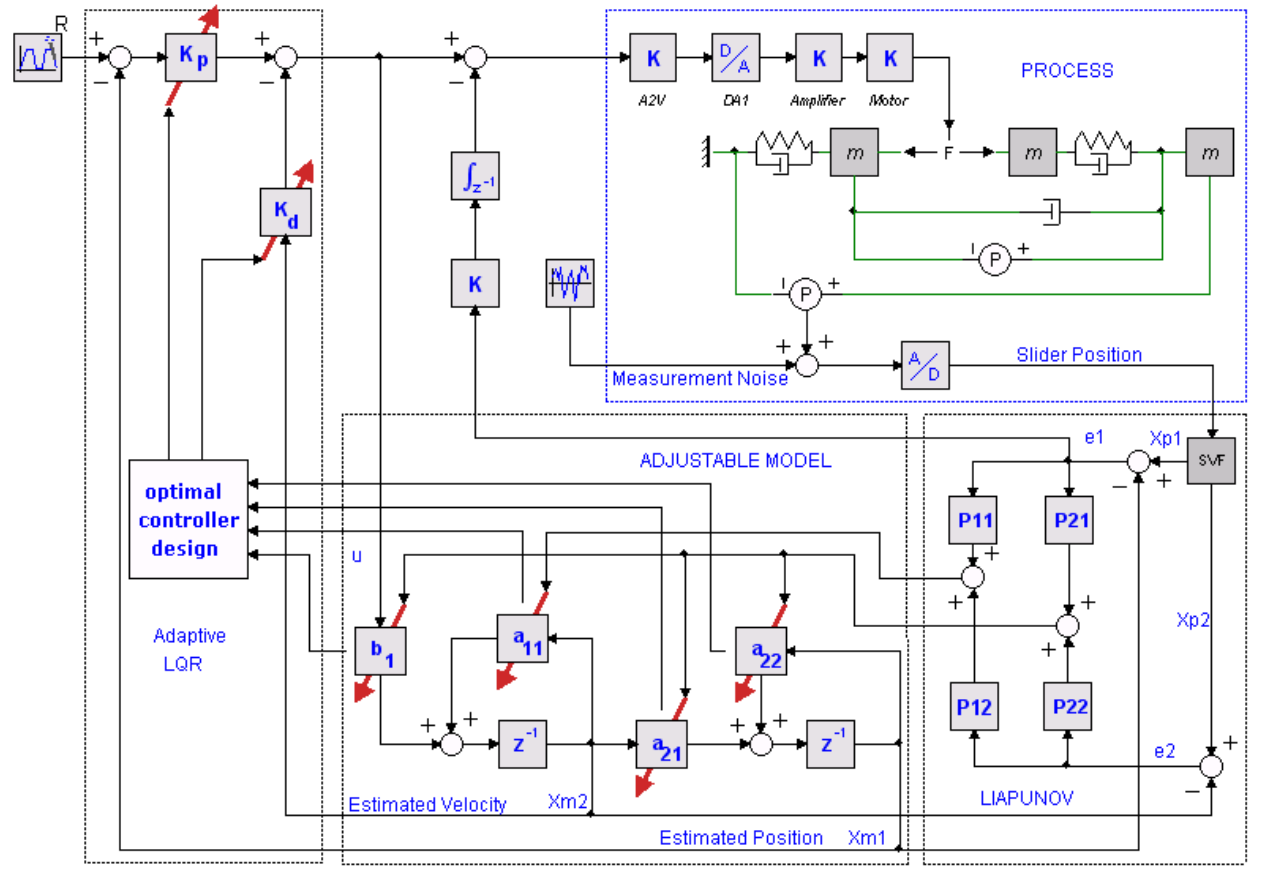

Figure 2.23: The Mede5 with the $2^{\text {nd }}$ adaptive observer and the $2^{\text {nd }}$ adaptive state feedback

The structure of the adjustable model depends on the chosen order, which is used for the identification. Here, we consider an adjustable model of second order. A state variable filter is used in order to obtain filtered values of the measured position and velocity. In discrete time the adjustable parameters of the adaptive observer are:

$$
\begin{gathered}
\left.b_{1}=\beta_{1} \sum\left[\left(p_{21} e_{1}+p_{22} e_{2}\right) u\right)\right] \cdot T_{s}+b_{1}(0) \\
a_{11}=\beta_{11} \sum\left[\left(p_{11} e_{1}+p_{12} e_{2}\right) x_{m 2}\right] \cdot T_{s}+a_{11}(0) \\
a_{21}=\beta_{21} \sum\left[\left(p_{21} e_{1}+p_{22} e_{2}\right) x_{m 2}\right] \cdot T_{s}+a_{21}(0) \\
a_{22}=\beta_{22} \sum\left[\left(p_{21} e_{1}+p_{22} e_{2}\right) x_{m 1}\right] \cdot T_{s}+a_{22}(0)
\end{gathered}
$$

in which $\beta_{1}, \beta_{11}, \beta_{21}$, and $\beta_{22}$ are the speeds of adaptation, $e_{1}, e_{2}, u, x_{m 1}$, and $x_{m 2}$ are defined in Fig 2.23, $T_{s}$ is the sampling interval, $p_{11}, p_{12}, p_{21}$ and $p_{22}$ are elements of the $P$ matrix, obtained from the solution of the Liapunov equation indicated in (2.27).

$$
\operatorname{next}(P)=\left(A_{p}^{T} P+P A_{p}+Q\right) \cdot T_{s}+P
$$




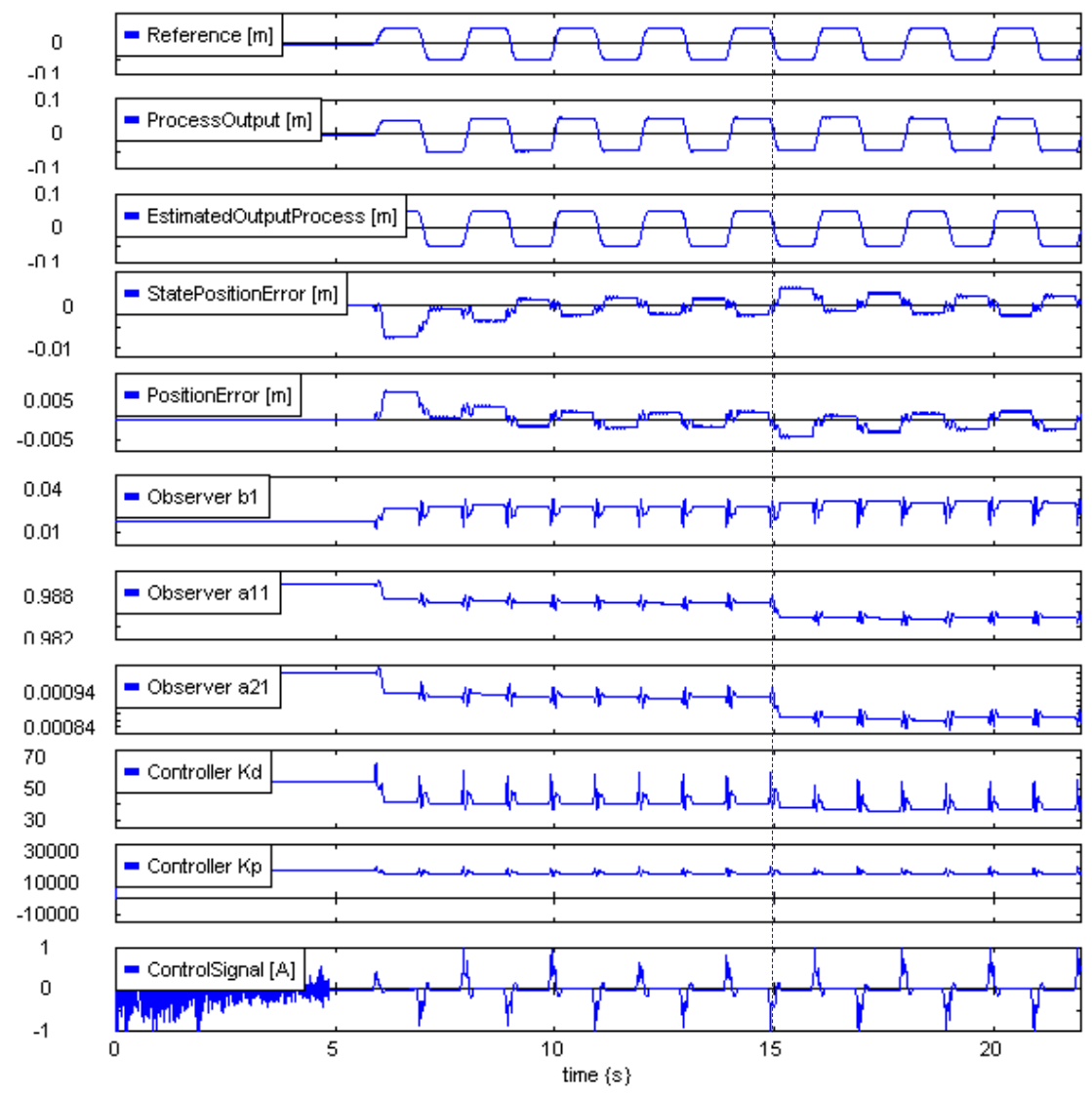

Figure 2.24: Simulation results of indirect MRAS controlled system (a load mass variation is added at $t=15[s]$ )

Because $A_{p}$ is not exactly known and may vary in time, we approximate $A_{p}$ by its nominal value (see Appendix A)

$$
A_{p}=\left[\begin{array}{cc}
-10 & 0 \\
1 & 0
\end{array}\right], B_{p}=\left[\begin{array}{c}
19 \\
0
\end{array}\right] .
$$

The following settings are used

$$
Q=\left[\begin{array}{cc}
100 & 0 \\
0 & 100
\end{array}\right], T_{s}=0.001
$$

The settings result in the stationary values of $P$

$$
p_{11}=0.9146, p_{12}=0.0043, p_{21}=0.0043, p_{22}=1.0000 \text {. }
$$




\section{Adaptive LQR}

The non-adaptive LQR design was shown in Sections 2.2 and 2.3. The gains of the controller are determined by minimizing a cost function, which reduces the tracking error and the control signal. When the state matrices $A$ and $B$ of the process are given accurately, the optimal feedback gain vector $K$ can be obtained. However, in practice an accurate state space description of the process is usually not present. And, if there is an initial accurate state space description but the process parameters change during operation, then the optimal feedback gain vector cannot be achieved. Adaptive LQR can be used in order to overcome this problem. In the control design, the feedback gains will be determined based on the $A_{m}$ and $B_{m}$ matrices of the adjustable model which follows continuously the process at different load conditions. The results of the combination of the adaptive LQR and the adaptive observer are illustrated in Fig 2.22. The detailed control structure based on the $2^{\text {nd }}$ order approximation of the $6^{\text {th }}$-order model is shown in Fig 2.23.

Fig 2.24 shows the corresponding responses for the system of Fig 2.23. The obtained responses show that the control structure was robust in the presence of sudden load changes and the control signal was relatively insensitive for measurement noise.

A Kalman filter has been shown to estimate the states and reject disturbances for systems with known parameters and when the injection points of all disturbances are known, while, the adaptive observer allows for the estimation and rejection of disturbances when the plant parameters are unknown. By comparing two simulation results as indicated in Fig 2.25left and Fig 2.25right, it can be seen that in the adaptive observer case, when a mass variation is added to the load, after a short time, the slider position state error $y_{p}-y_{m}$ converges rapidly to a small value. When the designer has limited knowledge of the plant parameters, it may be desirable to utilize MRAS to adjust the control law on-line in order to reduce the effects of the unknown parameters [40,22].
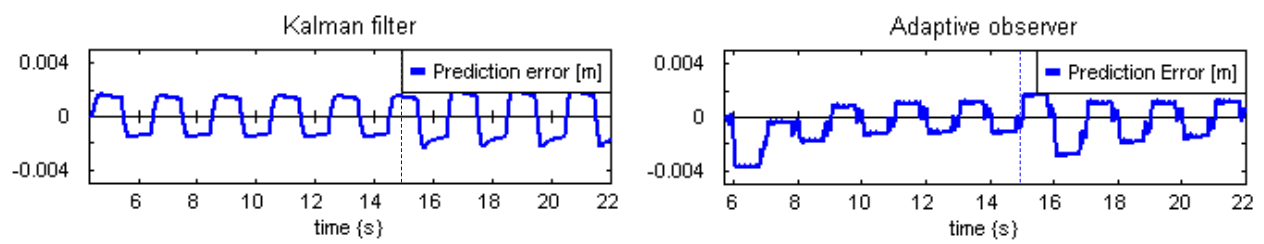

Figure 2.25: A comparison 


\subsection{Discussion}

A PID controller is an effective solution for most industrial control applications. It is often the first choice for a new controller design. The development went from mechanical devices to digital devices, but the control algorithm is almost the same [4]. The PID controller is used to make decisions about changes to the control signal that drives the plant. With the proportional action, the controller output can be adjusted by multiplying the error by a constant proportional gain. This gain is also frequently expressed as a percentage of the proportional band. The integral action gives the controller a large gain at low frequencies which results in reducing the static error. With the derivative action, the controller output is proportional to the rate of change of the error. It can stabilize loops since it adds phase lead. And it is often used to reduce overshoot.

The PID controlled system is sensitive to measurement noise. When the error is corrupted by noise, the noise content will be amplified by PID gains. Another problem with this controller is that the tracking error depends on plant parameter variations. Because the selection of PID gains depends on the physical characteristics of the system to be controlled, there is no set of constant values that is suitable to every implementation when the dynamic characteristics are changing. In fact, the control designer wants to reach a controlled system which is insensitive for measurement noise and plant parameters variations. Insensitive for measurement noise suggests low PID gains. While, insensitive for plant parameter variations suggests high PID gains. Achieving both desired properties at the same time may not be possible in every system. Negative points of the conventional PID controller, can be solved by applying more advanced controllers.

For linear finite-dimensional systems, LQR theory plays a particular role because optimal gains can be easily calculated by solving algebraic Riccati equation and the control signal stabilizes the closed-loop system. The LQR design is to find a state-feedback law that minimizes a cost function, which involves the desired performance specifications of the closed loop system. The cost function is a quadratic performance criterion with user-specified weighting matrices. The optimal state feedback requires full-state measurements of the plant to be controlled. In practice, however, not all state variables are available for feedback. In addition, the measured state variables may be corrupted by measurement noise at any time instant. The Kalman filter is a common approach to overcome these problems.

The Kalman filter is an efficient recursive filter that supports estimation of past, present, and even future states of a dynamic system when dealing with Gaussian white noise. It minimizes the asymptotic covariance of the estimation error. In optimal control theory, the Kalman filter is known as Linear Quadratic Estimation (LQE). The choice of the process noise covariance $Q_{E}$ and the sensor noise covariance $R_{E}$ has a great influence on the optimal state filter gain $L$. The solution 
is a compromise between model and sensor uncertainty.

The combination of the optimal LQR and LQE results in the LQG controller, which is optimal under the given quadratic cost function. The estimator and feedback controller may be designed independently. It enables us to compromise between regulation performance and control effort, and to take into account process and measurement noise. However, it is not always obvious to find the relative weights between state variables and control variables. Most real world control problems involve nonlinear models while the LQG control theory is limited to linear models. Even for linear plants, the mathematical models of the plants are subject to uncertainties that may arise from un-modeled dynamics, and parameter variations. These uncertainties are not explicitly taken into account in the LQG design. These problems can be solved, by using adaptive control systems such as Model Reference Adaptive Systems (MRAS) or Self Tuning Regulators (STR).

The basic philosophy behind Model Reference Adaptive Systems (MRAS) is to create a closed loop controller with parameters that can be adjusted based on the error between the output of the system and the desired response from a reference model. For implementing MRAS when only input and output measurements are available, state variable filters (SVFs) can be used, allowing us to obtain filtered derivatives. Moreover, the SVFs offer a beneficial solution to reduce the influence of the measurement noise if the noise spectrum is principally located outside the band pass of the SVFs. Direct MRAS offers a potential solution to reduce the tracking errors. However, this control algorithm may fail to be robust to measurement noise. In indirect MRAS, estimation of parameters in the model leads indirectly to adaptation of parameters in the controller. In other worlds, for indirect MRAS the adaptation mechanism modifies the system performance by adjusting the parameters of the adjustable model, by adapting the parameters of the controller. Indirect MRAS can have a beneficial effect on reducing the influence of measurement noise. On line MRAS is vital for control systems where the model parameters are poorly known, due to modeling errors and changing environment. However, the MRAS methods need a priori structural information of the plant to be controlled. 


\section{Chapter 3}

\section{Neural Network based Learning Feed-Forward Control}

\subsection{Introduction}

In order to eliminate positional inaccuracy due to reproducible disturbances and model uncertainty we consider a learning feed-forward controller structure that consists of a feedback and a feed-forward controller. We assume that the state of the process and the state of the reference model are identical and use the approximated inverse dynamics of the process to compute the feed-forward signal. For proper reference signals and when there are no disturbances, if the feed-forward controller equals the inverse of the plant, the tracking error will be zero. The feedback controller is designed such that robust stability is guaranteed in the presence of model uncertainty, while the feed-forward controller is used to compensate for known reproducible disturbances.

The aim of this chapter is the design of the feed-forward controller. The problem that we are going to solve is to find a suitable feed-forward structure and parameters that allow achieving an as small as possible tracking error with respect to the reference input, i.e. the error between the output of the reference path generator and the end-effector (see Fig1.1). We would like to note the contributions of De Vries et al $[10,9,34,11,12,13,46,45]$ as a motivation for the development of learning feed-forward control. The design procedure in here is also based on their published papers.

This chapter consists of ten main sections. In the next section a brief definition of learning control is given. Feedback error learning (FEL) is introduced in Section 3.3. Section 3.4 presents a learning feed-forward controller (LFFC). In Section 3.5 we discuss in detail how to select the inputs for the feed-forward part. The B-spline distribution on each of the inputs of the feed-forward part is 
introduced in Section 3.6. The concepts "Curse of Dimensionality in LFFC" and "Parsimonious LFFC" are discussed in Section 3.7. Phase correction for LFFC is introduced in Section 3.8. Section 3.9 treats the design of a parsimonious LFFC. The obtained knowledge is used to formulate a practical design procedure. The effectiveness of the proposed controller is illustrated through the simulation results. At the end of the chapter a review is given.

\subsection{Learning Control}

Traditionally, the design of a controller is often based on an explicit plant description in terms of a mathematical model [15]. The mathematical model, which describes the dynamical behavior of the plant, is firstly developed, and then control design techniques are applied to design appropriate controllers. The mathematical model of the system must be "simple enough" so that it can be analyzed with available mathematical techniques, and "accurate enough" to describe the important aspects of the relevant dynamical behavior [33]. The controller is then realized via hardware and/or software and it is used to control the physical system. It is clear that, a more accurate mathematical model of the plant will result in a controller with a better performance. However, in many practical cases the mathematical model of the plant to be controlled is generally unknown or ill defined. Moreover, the system may be complex with non-linearity and parameter variation problems. When accurate mathematical models are unavailable or too complex to be established, learning control may be considered [12,45].

Neural networks offer methodologies to perform learning functions. A learning controller comprises a function approximator of which the input-output mapping is adapted during control such that a desired behavior of the controlled system can be obtained. A function approximator is an input-output mapping determined by a selected function $F(., \theta)$, of which the parameter vector $\theta$ is chosen such that a function $f($.$) is "best" approximated [32].$

Neural network literature for function approximation is sufficiently wealthy [24]. In its complete form, the problem involves both parametric and structural learning. The properties of a neural network are determined by its structure and the learning rule is used to adapt the weights. The network structure is defined based on the basic processing units and the way in which they are interconnected. The network allows any unit to be connected to any other unit in the network. The principal importance of a neural network is not only the way a neuron is implemented but also how interconnections are made. The learning rules, also known as training algorithms, are procedures for modifying the weights on the connection links in a neural network. Finding appropriate learning rules and finding an appropriate network structure are two different issues. Neural networks typically take a 
vector of input values and produce a vector of output values. Inside, the weights of neurons are trained to build a representation of a function that will approximate the input to output mapping.

\subsection{Feedback-error learning using multi-layer per- ceptron neural network}

\subsubsection{Control Structure}

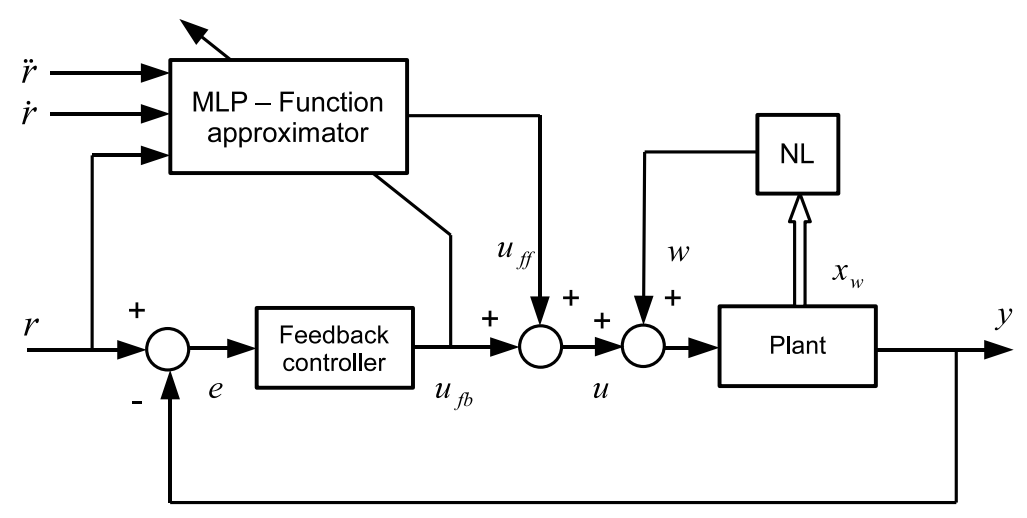

Figure 3.1: Feedback-error learning control; A Multi-layer perceptron neural network is used in the feed-forward part.

Fig 3.1 shows the block diagram of a feedback-error learning (FEL) controller as introduced in [19], which combines a feed-forward controller and a linear feedback controller. The type of function approximation that Kawato used is a Multi Layer Perceptron (MLP) neural network. Among various neural network learning control schemes, feedback error learning has been known for the reason that it has advantages over other schemes $[26,45]$. This technique aims to acquire the inverse dynamics model of the plant and uses neural networks acting as an adaptive controller to minimize the tracking error during training. Most control action for disturbance rejection is finally performed by the feed-forward action. The FEL can also be interpreted as a form of nonlinear adaptive control [26].

Commonly, a few simple types of feedback controller structures such as PD, PID, or PID plus a low pass filter are applied. In general, the feedback controller is used to provide the learning signal for the feed-forward controller, and to robustly stabilize the system. Furthermore it determines minimum tracking performance at the beginning of learning 


\subsubsection{Plant disturbance compensation}

Motion control systems are usually designed to track given trajectories with small tracking errors. The assumed model of a plant with state dependent effects is illustrated in Fig 3.2. There are only integrators in the forward path. The state dependent effects are assumed to be known and act on the input that generates effects which are known as reproducible disturbances. Thus, reproducible disturbances can be viewed as a function of the state of the plant. These disturbances may significantly affect the control performances during the object operations.

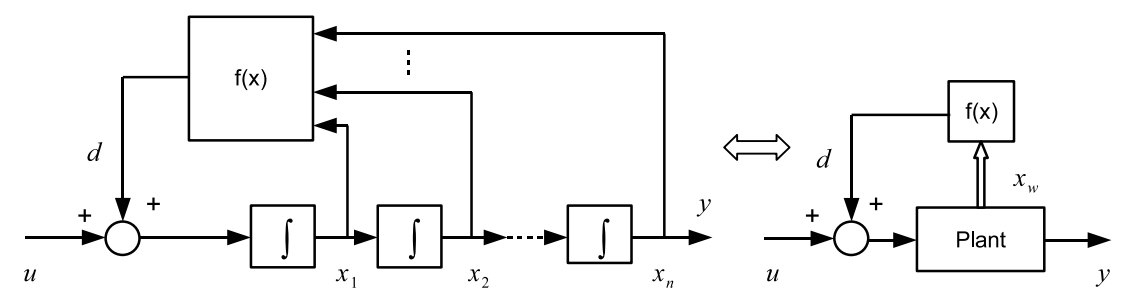

Figure 3.2: Plant and reproducible disturbances.

The reproducible disturbances would be difficult to be compensated by mechanical or electrical means. However, a fast and accurate servo response can be achieved by using a state space reference model with feed-forward compensation of these disturbances. If the disturbances are known then a control signal that attempts to counteract the disturbances can be created and applied to the process. For example: The inertia of a rotating mass can be computed or measured. If the inertia and the desired acceleration rate are known, the torque required to accelerate the mass to a desired speed can be computed and then used as a feed-forward signal such that the rotational speed tracks the speed reference with a small error. This is known as inertia compensation. In order to achieve high-precision motion control, friction must be appropriately compensated for. Coulomb friction has a constant value with a sign, which is opposite to the sign of the velocity. Viscous friction is proportional to the velocity. The total friction force can be modeled as a velocity-dependent nonlinear disturbance. The friction force can be predicted largely if the velocity is known and can thus be effectively compensated. The cogging force is considered as a position-dependent disturbance. It is generally modeled as a sinusoidal function of the displacement. Therefore, if the feed-forward part has access to the desired position, the cogging disturbance can be compensated. 


\subsubsection{Multi-Layer Perceptron (MLP) neural network}

MLPs are the most common neural network structures used for a broad range of applications [26, 31]. A multi-layer neural network is a neural network, which has more than one layer of neurons. The first layer is called the input layer, with a number of neurons equal to the number of input variables of the problem. The last one is the output layer, where the perceptron response is made (a single-layer neural network is called a perceptron), with a number of neurons equal to the desired number of quantities computed from the inputs. In between there may be one or more hidden layers. Neurons in the hidden layer compute a non-linear function of their input (with no hidden layer, the perceptron can only perform linear tasks). Every processing node in one particular layer is usually connected to every node in the layer above and below.

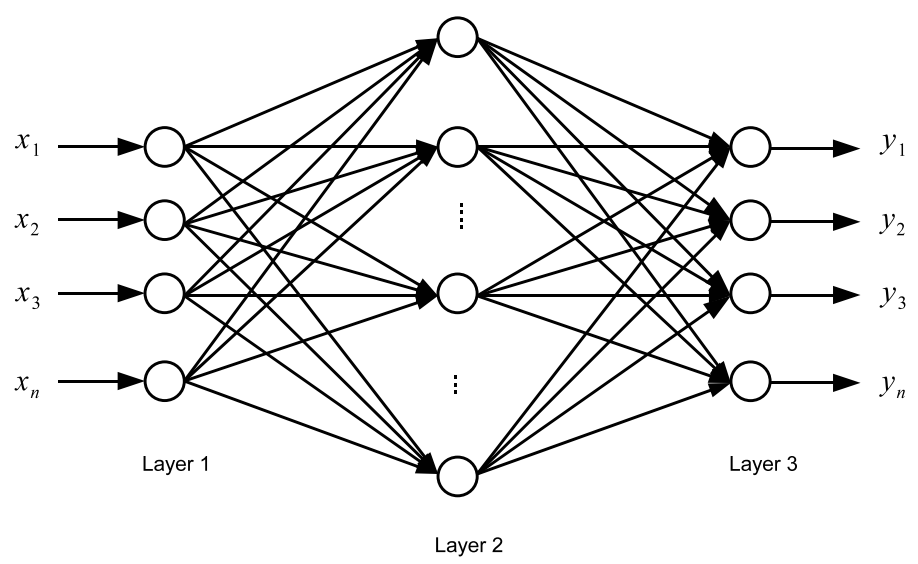

Figure 3.3: Multi Layer Perceptron Network with one hidden layer (the weight of each connection is not represented but must exist in the reality).

Training the MLP networks is performed, by adapting the weights of the connections according to the back propagation mechanism [31]. The connections carry weights, which encapsulate the behavior of the network. Beginning with small random weights, the linear neurons are adjusted so that a slight non-linearity is added after the linear summation. The training set is presented iteratively to the network until a stable set of weights is achieved and the error between the network output and the desired output is reduced to an acceptable level. The complexity of the MLP network can be changed by varying the number of layers and the number of units in each layer. In fact, it has been shown that, given enough hidden units and enough data, MLPs can approximate almost any function to any desired accuracy [26]. This means that MLPs network are universal function approximators.

In the design of the feedback error learning the following advantages of MLP 
networks can be shown [31, 24]: first, the MLP network exhibits a great degree of robustness and fault tolerance because of built-in redundancy. Second, the MLP network can form any unbounded decision region in the space spanned by the inputs. Third, the MLP networks have a capability for function approximation. However, the MLP network has the following disadvantages: first, it is often unclear which network topology is required for the solution of a given task. For a given problem, the numbers of hidden units are tuned until satisfactory results are obtained. Second, the network might become trapped in a local minimum of the error function being minimized. This can be caused by an improper setting of the initial weights. Third, there is no theory explaining the behavior and mapping capabilities of the MLP network. There is also no rule to determine the optimal number of hidden units required for the solution of a given task. Moreover, the method to find a set of weights to approximate the given function is still unclear. To overcome these problems, in Learning Feed-forward Control introduced later, a B-spline network is chosen for the feed-forward part instead of an MLP.

\subsection{Learning feed-forward control using B-spline neural network}

\subsubsection{Control structure}

Since the difficulties of FEL control are mainly caused by the MLP network, one of the approaches to overcome the problems is the use of a B-spline network for the feed-forward part instead of an MLP.

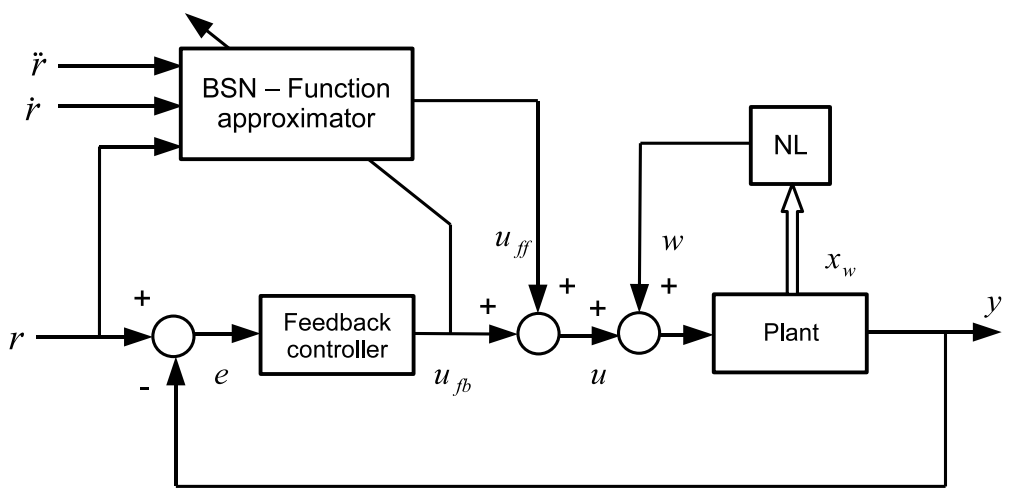

Figure 3.4: Learning Feed-Forward Control; A B-spline neural network is used in the feed-forward controller.

This adopts direct inverse control of B-spline neural network. The neural net- 
work inverse model of the plant is in series with the plant such that the transfer function between the input of the system and the output of the plant is equal to 1. In order to obtain the input-output behavior of an inverse dynamics model over time, the B-spline network should be trained. During training, the plant output is compared with a desired reference output. The error between these two signals is used to adjust the weights which are known as the free parameters of the function approximator.

\subsubsection{B-spline neural network}

The B-spline neural network (BSN) has become popular, mainly due to its ability to universally approximate any continuous nonlinear function $[12,24]$. In a BSN the input-output mapping is created using basis functions, known as B-splines. A B-spline of order $n$ consists of piece-wise polynomial functions of order $n-1$. The function evaluation of a B-spline is generally called the membership and is denoted as $\mu \in[0,1]$. That part of the input space for which $\mu \neq 0$ is known as its support. The output of the BSN is a weighted sum of the B-spline evaluations.

We will only consider B-splines of order 2. In order to produce an input-output mapping from $r, \dot{r}$ and $\ddot{r}$ to $y$, the B-splines are placed on the domain of the BSN input such that at each input value the sum of all memberships equals 1 . The output of the BSN is determined as

$$
y(x)=\sum_{i=1}^{N} w_{i} \mu_{i}(x),
$$

in which $y(x)$ is the output of the BSN, $\mu_{i}(x)$ is the membership of the $i-t h$ basic function at input $x, w_{i}$ the weight of the basis function, and $N$ is the number of B-splines. Training of the network can either be done after each sampling interval, which is known as on-line learning, or after a motion has been completed, known as off-line learning. In the on-line case, the cost function $j$ that is minimized is the squared approximation error between the desired output of the BSN $y_{d}$ and the actual output $y[24,45]$ :

$$
J=\frac{1}{2}\left(y_{d}-y\right)^{2}
$$

Applying the back propagation rule, this yields:

$$
\Delta \omega_{i}=-\gamma \frac{\partial J}{\partial \omega_{i}}=\gamma\left(y_{d}-y\right) \frac{\partial y}{\partial \omega_{i}}
$$

Substituting (3.1) in (3.3) gives:

$$
\Delta \omega_{i}=\gamma\left(y_{d}-y\right) \frac{\partial \sum_{j=1}^{N} \mu_{j}(x) \omega_{j}}{\partial \omega_{i}}=\gamma\left(y_{d}-y\right) \mu_{i}(x)
$$




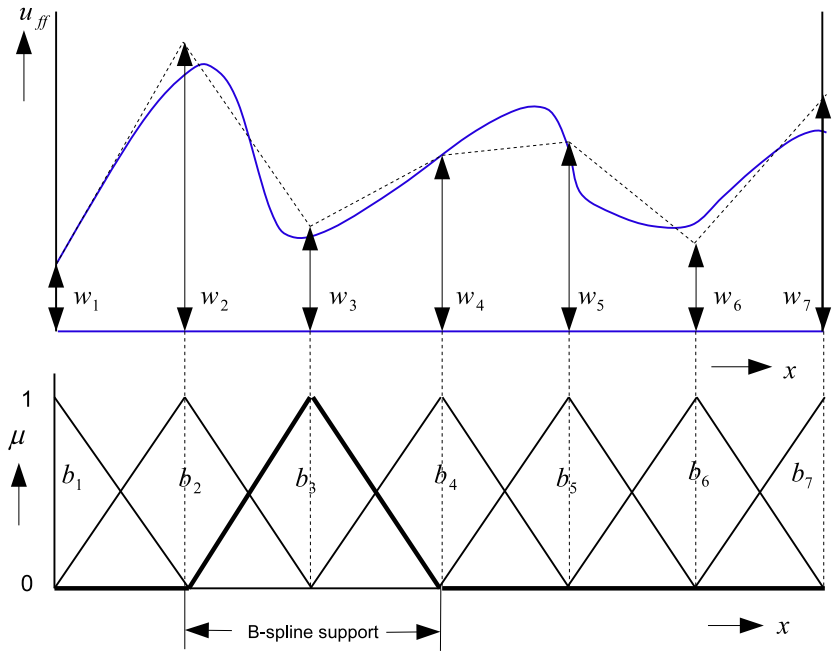

Figure 3.5: Top: I/O mapping. Bottom: $2^{\text {nd }}$ order B-splines.

In this equation $\Delta \omega_{i}$ denotes the adaptation of the weight of the $i-t h \mathrm{~B}$-spline, and $\gamma$ the learning rate, $0<\gamma<1$. In the off-line case the cost function is given by:

$$
J=\frac{1}{2} \sum_{j}\left(y_{d, j}-y_{j}\right)^{2},
$$

where $y_{d, j}$ is the desired output for input $x_{i}$ and $y_{j}$ the actual output of the BSN. The adaptation of weights is then determined as:

$$
\Delta \omega_{i}=\gamma \sum_{j}\left(y_{d, j}-y_{j}\right) \mu_{i}^{n}\left(x_{j}\right)
$$

In order to prevent large weight-adaptations, (3.6) is normalized by:

$$
\Delta \omega_{i}=\gamma \frac{\sum_{j}\left(y_{d, j}-y_{j}\right) \mu_{i}^{n}\left(x_{j}\right)}{\sum_{j} \mu_{i}^{n}\left(x_{j}\right)}
$$

The off-line learning rule (3.7) implies that the weights are updated after a reference motion has been completed.

\subsubsection{Design of LFFC using BSNs}

In the detailed design of LFFC the following parameters of the feed-forward part have to be considered based on stability analysis in the frequency domain [46]: 
1. Selection of the inputs of the feed-forward part: The inputs of the BSN depend on the nature of the plant and the reproducible disturbances that the LFFC has to compensate. For random motions, the inputs should consist of the reference position and its derivatives/integrals.

2. Selection of the B-spline distribution on each of the inputs of the BSN: The BSN is able to accurately approximate a given signal by choosing a suitable width of the B-splines. A large number of "narrow" B-splines, allows the BSN to precisely approximate a high frequency signal. While a low frequency target signal requires a small number of "wide" B-splines.

3. The learning mechanism: Adaptation of the network weights can either be done after each sample, which is known as on-line learning, or after a motion has been completed, known as off-line learning.

4. The learning rate: The learning rate $\gamma$ determines how strong the weights of the BSN are adjusted. A large learning rate implies that the weights are adjusted quickly. When a small learning rate is used the weights are adjusted slowly. However, a too high learning rate will cause unstability.

By using a BSN, we have the following advantages [45]:

1. Local learning: Due to the compact support of the B-splines, only a small number of the weights contribute to the output. Only these weights need to be updated during training. While, in the case of the MLP, during training all network weights need to be updated. This means, the BSN converges much faster than the MLP.

2. No local minima: The output of the BSN is a linear function of the weights. This implies that, both for off-line and on-line learning the learning mechanisms do not suffer from local minima. This means that the initial weights of the BSN do not influence the final tracking accuracy.

3. Tune-able precision: The choice of the number of the B-splines and their locations determines the smoothness of the input-output relation. Random target signals can be accurately approximated, by choosing B-splines that have a proper compact support.

The principal drawback of BSNs is that the number of network weights grows exponentially with the dimension of the input space [12]. 


\subsection{Input selection of the LFFC}

Models of the plant dynamics and of the reproducible disturbances are needed to create a structure for non-repetitive motions. We assume that the process can be modeled by a linear state space model and additive non-linear dynamics, shown in Fig 3.6. This yields for the linear process

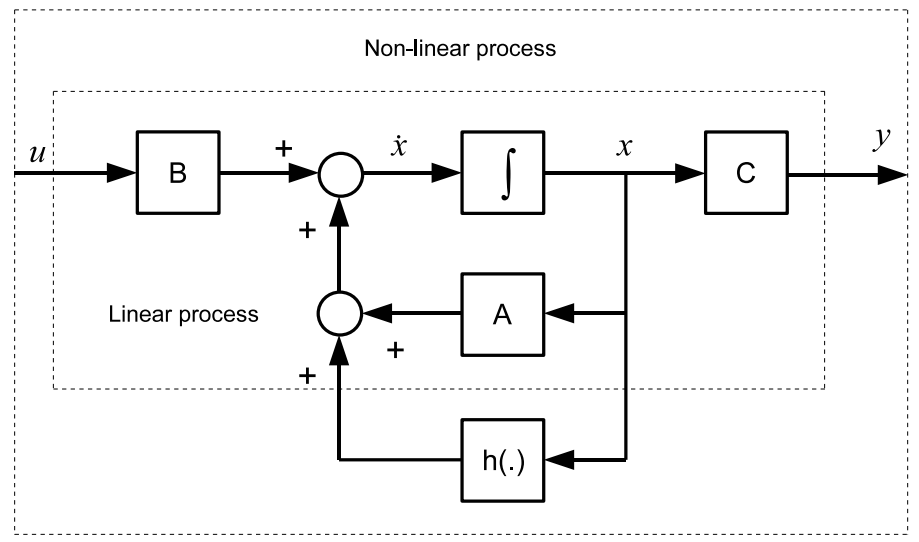

Figure 3.6: A non-linear process consists of a linear process and a non-linearity $\mathrm{h}($.$) .$

$$
\dot{x}=A x+B u
$$

The state vector is chosen such that it consists of positions $\left(x_{2}\right)$ and their corresponding velocities $\left(x_{1}\right),\left(\dot{x}_{2}=x_{1}\right)$. This gives

$$
\left[\begin{array}{l}
\dot{x}_{1} \\
\dot{x}_{2}
\end{array}\right]=\left[\begin{array}{cc}
A_{11} & A_{12} \\
I & 0
\end{array}\right]\left[\begin{array}{l}
x_{1} \\
x_{2}
\end{array}\right]+\left[\begin{array}{c}
B_{1} \\
0
\end{array}\right] u
$$

Now we consider additive non-linear dynamics. For example, the case that the process has both a velocity and a position dependent non-linearity. This yields for the non-linear process:

$$
\left[\begin{array}{c}
\dot{x}_{1} \\
\dot{x}_{2}
\end{array}\right]=\left[\begin{array}{cc}
A_{11} & A_{12} \\
I & 0
\end{array}\right]\left[\begin{array}{l}
x_{1} \\
x_{2}
\end{array}\right]+\left[\begin{array}{c}
h\left(x_{1}\right)+h\left(x_{2}\right) \\
0
\end{array}\right]+\left[\begin{array}{c}
B_{1} \\
0
\end{array}\right] u
$$

The desired feed-forward signal $u_{d}$, that makes the actual position of the process $x_{2}$ track the desired states $x_{2, d}$, is given by (if $B_{1}^{-1}$ exists):

$$
B_{1}^{-1}\left(\ddot{x}_{2, d}-A_{11} \dot{x}_{2, d}-A_{12} x_{2, d}-h(.)\right)=u_{d}
$$

The specific inputs of a BSN can be determined on the basis of a (non-linear) state space representation of the plant dynamics. To ensure the LFFC to accurately control the process for random motions, the states involved in (3.11) should be used as 
inputs of the BSNs. In general, this will result in a BSN that has multiple inputs. It is clear that when the disturbances depend on the velocity of the plant, the reference velocity must be used as input of the LFFC. In case the disturbances do not only depend on the velocity of the plant, but also on the acceleration, both the reference velocity and acceleration must be used as inputs of the LFFC. Each of the reference inputs of the LFFC will be used to compensate one specific disturbance.

\subsection{B-spline distribution}

The aim of the BSN is to create the control signal that would result in the smallest possible tracking error. The width or support of the B-splines determines the accuracy such that the BSN is able to approximate an input-output relation. In case a one-dimensional BSN with second-order B-splines is used, the output consists of first-order polynomials between the B-spline knots [45]. Therefore the smaller the distance between the B-spline knots the more accurate the LFFC can be obtained. When a small width is chosen the BSN is able to accurately approximate high frequency signals. For smooth target signals, a large width of the B-splines is required. The width of the B-splines should be chosen such that the BSN is able to learn the desired feed-forward signal.

As stated the accuracy of the approximation can be improved if a large number of the B-splines is applied. The designer would naturally prefer a distribution that has a large number of the B-splines. In this case the BSN is able to accurately learn any feed-forward signal. However, the following troubles may happen [45]: firstly, the stability analysis shows that B-splines with a small width may result in unstable behavior. Secondly, the rate of convergence of the LFFC decreases. Finally, a large amount of computer memory is required to store the feed-forward signal.

Prior knowledge of the process and the disturbances are needed to determine the width of the B-splines. The minimum width of the B-splines, $d_{\min }$, for which the system remains stable, can be determined on the basis of a Bode plot of the negative complementary sensitivity function, $|-T(j \omega)|_{\infty}$, of the closed loop. The following steps are given [46]:

1. Determine $|-T(j \omega)|_{\infty}$ from a model of the closed loop system.

2. Use the Bode plot of the model to find out $\min _{\{\omega \in R \mid \cos (\varphi) \leq 0\}}|-T(j \omega)|$

3. Search for the smallest value of $\omega_{1}$ at which $\varphi_{1}=\arg \left(-T\left(j \omega_{1}\right)\right.$ satisfies

$$
\varphi_{1}=\arccos \left[-0.0147 \cdot \frac{|-T(j \omega)|_{\infty}}{\min _{\{\omega \in R \mid \cos (\varphi) \leq 0\}}|-T(j \omega)|}\right]
$$


4. The minimum value of the width of the B-splines, $d_{\text {min }}$, is given by

$$
d_{\text {min }}=\frac{2 \pi}{\omega_{1}}[\sec ]
$$

Formula 3.13 can be used to derive e.g. speed limitation that should be obeyed during training of position-dependent disturbances. Later, we show how this can be done.

In order for the learning to converge, the learning rate should satisfy [45]

$$
\gamma \leq \frac{2}{|-T(j \omega)|_{\infty}}
$$

When the minimum width of the B-splines has been determined, then the distribution of the B-splines can be chosen. The B-spline distribution should be determined on the basis of the desired reference inputs which are supposed to be known. Based on the knowledge of these inputs, an initial B-splines distribution is chosen. However, the initial B-spline distribution may not result in a satisfactory tracking performance. To optimize the tracking performance, the B-spline distribution could be adjusted in an iterative way during the training phase. This is implemented, by decreasing the width of the B-splines at those positions where the tracking performance is poor [45]. Because $-T(j \omega)$ depends on the feedback controller, it follows that if the obtained value $d_{\min }$ is too large, the feedback controller should be redesigned.

\subsection{Parsimonious LFFC}

The BSN is trained on the basis of observed input-output data. When the inputs of the LFFC are chosen in a straightforward way on basis of qualitative knowledge of the plant model and the disturbances, this often leads to BSNs that have multiple inputs. For a B-spline the number of the network weights depends on the dimension of the input space in an exponential way. The growth of the number of weights will result in large memories, poor training, and bad generalization. This is known as the curse of dimensionality. To overcome this problem, a network structure should be chosen that it consists of several low-dimensional BSNs instead of one high-dimensional BSN, each compensating for one specific disturbance. This is known as a parsimonious network structure [11]. The parsimonious LFFC generates a feed-forward signal by summing the BSNs output signals with properly chosen weights.

To make the problem clear, we consider an example

$$
y=f\left(x_{1}, x_{2}, x_{3}\right)=f_{1,2}\left(x_{1}, x_{2}\right)+f_{2}\left(x_{2}\right)+f_{1,3}\left(x_{1}, x_{3}\right)
$$


This function can either be learnt by one three-dimensional network or by a parsimonious network structure consisting of three lower-dimensional BSNs, see Figure 3.7. If the above function would be learnt in one three-dimensional network,

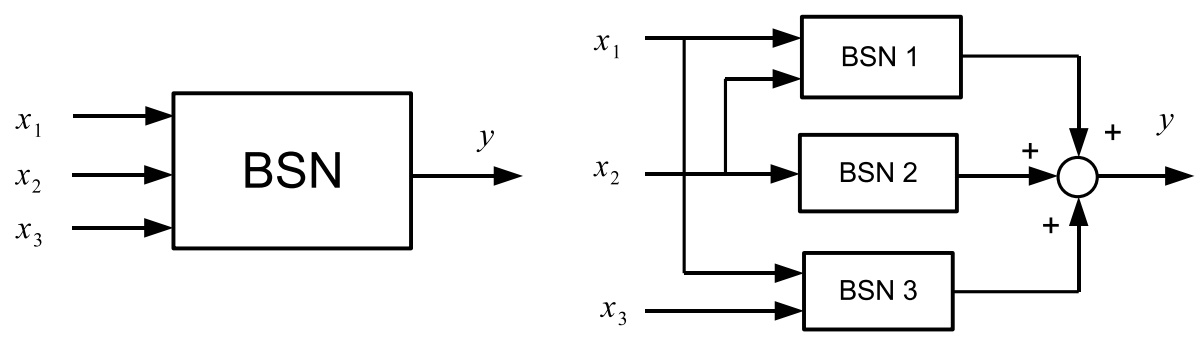

Figure 3.7: Splitting up one multi-dimensional SBN (left) into several lower-dimensional BSNs (right).

the total number of weights would be equal to

$$
N_{\text {multi }}=N_{1} N_{2} N_{3},
$$

where $N_{i}$ is the number of weights of one-dimensional BSN defined on the input domain. Assuming $N_{1}=N_{2}=N_{3}=10$ this results in $N_{\text {multi }}=1000$. However, if this function would be learnt in a network consisting of three lower-dimensional networks, the total number of required weights would become

$$
N_{\text {lower }}=N_{1} N_{2}+N_{2}+N_{1} N_{3}=210 .
$$

\subsection{Phase correction for LFFC}

\subsubsection{Un-delayed learning}

We first consider the ideal case. In Figure 3.8 (left) a block diagram is given of the system which will be used as an example. We use a B-spline network with one input to approximate a simple non-linear function from the reference model:

$$
y_{p}=\sin (\text { input })
$$

where the input $=A \sin (\omega t), A=1[\mathrm{~m}]$, and $\omega=20[\mathrm{rad} / \mathrm{s}]$. A network with 10 second-order splines is used. This B-spline network is trained by adapting the weights. During training the network output is compared with a desired output. The actual error $e$ between these two signals is used to adapt the weights. The output of the B-spline network is a combination of all ten B-Splines. The simulation results when the actual error is being used to adapt the weights is illustrated in Fig 3.9 (left). As can be seen, the error rapidly decreases and converges after a few runs, due to the network learning. 

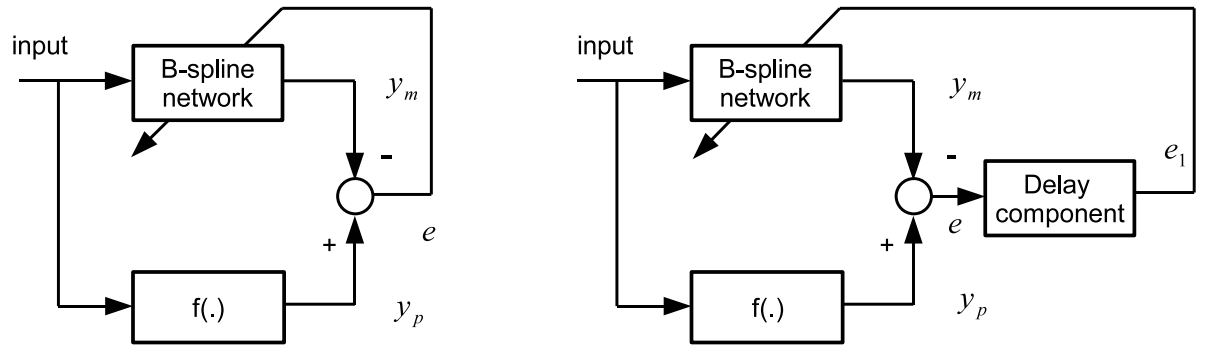

Figure 3.8: Left: un-delayed learning. Right: delayed learning learning.
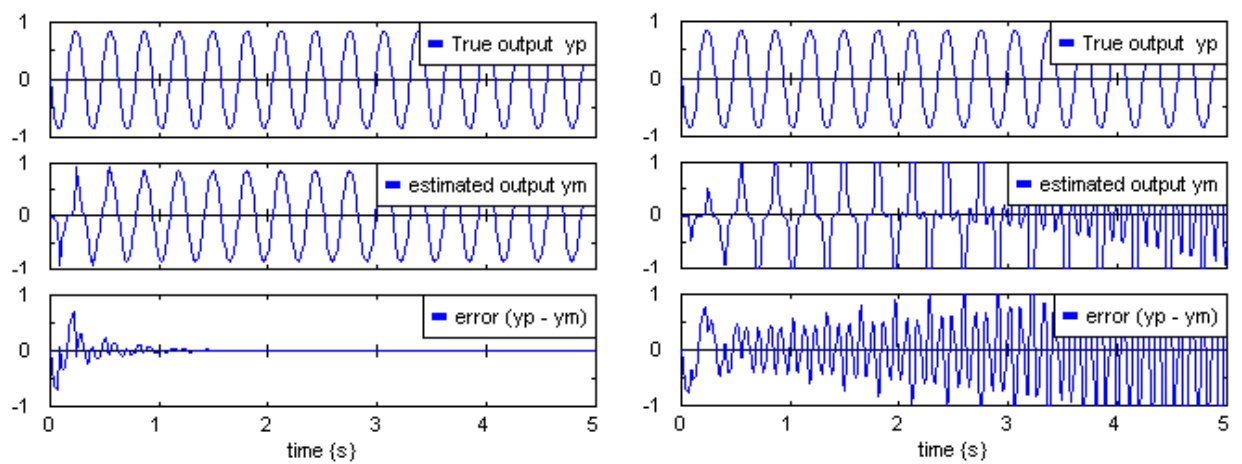

Figure 3.9: Simulation results: (left) un-delayed and (right) delayed learning.

\subsubsection{Delayed learning}

However, the model shown in Fig 3.8 (left) is an ideal case because in practice the actual error $e$ cannot be directly measured. We can only get information about $e$ delayed by plant dynamics. This indicated in a simplified form in Fig 3.8. The signal $e_{1}$ is available instead of $e$. The simulation results due to the delayed error being used to adapt the weights as illustrated in Figure 3.9 (right). In this simulation a low pass filter is chosen as the delay component with the transfer function:

$$
H(s)=\frac{\omega_{n}^{2}}{s^{2}+2 \varsigma \omega_{n} s+\omega_{n}^{2}}
$$

The following numerical values are chosen:

$$
\omega_{n}=50, \varsigma=2 .
$$

From the obtained simulation results, it is observed that the error rapidly increases and diverges in the next runs. 


\subsubsection{Phase-corrected learning feed-forward control}

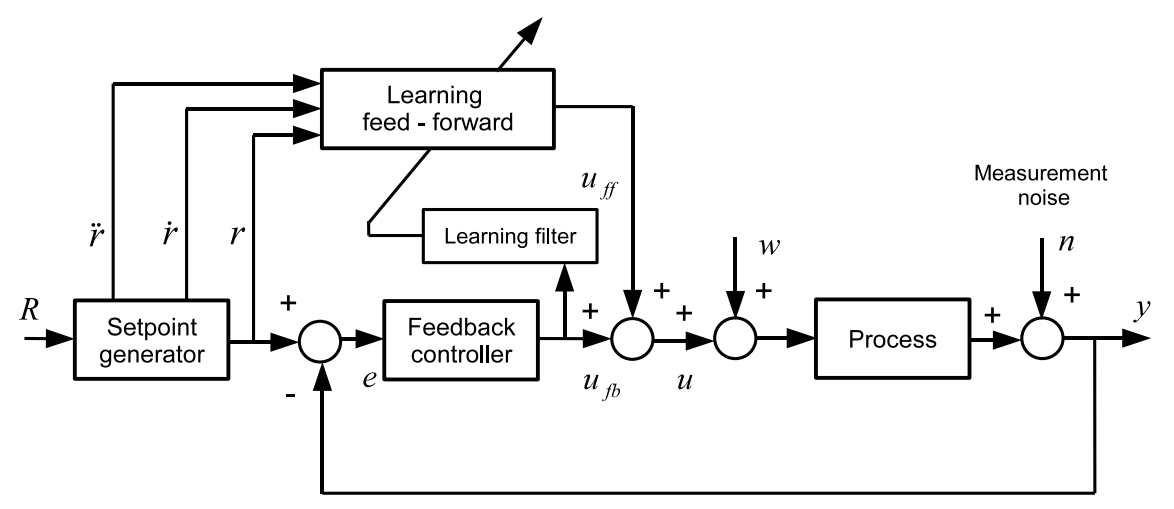

Figure 3.10: Learning filter is used to generate the learning signal.

The phase shift results in growth of the tracking error. Adding $T^{-1}$ ( $T$ denotes the complementary sensitivity function) in series with the learning signal offers a solution. Instead of using the output signal from the feedback controller, a filtered signal is used to adapt the weights (see Fig 3.10). The learning filter $F$ is designed in such way that it corrects the phase shift of the closed loop transfer function. The transfer function of $T^{-1}$ is given by:

$$
T_{(s)}^{-1}=\frac{1+C_{(s)} P_{(s)}}{C_{(s)} P_{(s)}}
$$

However, this filter is not proper. Adding a low-pass filter $Q$ offers a solution to this. The transfer function of $Q$ is given as:

$$
Q(s)=\frac{\omega_{n}^{2}}{s^{2}+2 \varsigma \omega_{n} s+\omega_{n}^{2}}
$$

The value of $\omega_{n}$ will usually be somewhat large than the bandwidth of T. The transfer function of the learning filter is determined by:

$$
F(s)=T_{(s)}^{-1} Q(s)
$$

Note that application of this filter implies that a smaller $d_{m}$ is obtained (see 3.13).

\subsection{Design of a parsimonious LFFC}

In this section a parsimonious LFFC will be designed on the basis of the analyses shown in the previous sections. The controlled process is the MeDe 5 that has been 
described in Chapter 1. An approximate model, used in the simulations, is shown in Fig 3.11. As stated in Chapter 1, for the MeDe5, the cogging phenomenon is so insignificant that can be ignored. However, in order to illustrate the effectiveness of the control method, the cogging force is added in the plant model. The plant state vector is chosen such that it consists of the position and its corresponding velocity. The LFFC is to learn the steering signal $u$, which makes the actual position of the process $y$ track the desired reference position $r$ with a small tracking error. The design procedure for a parsimonious LFFC is give below:

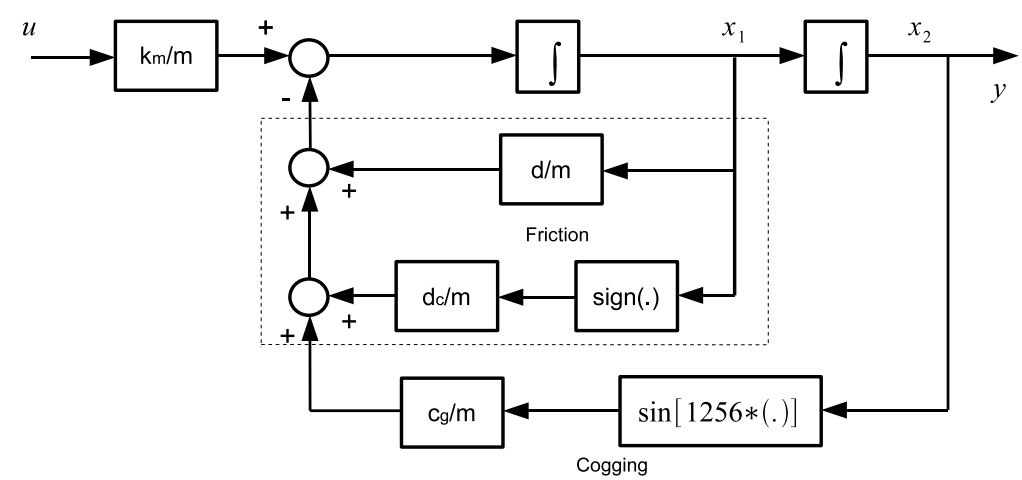

Figure 3.11: Approximate model of the MeDe5.

\section{Step 1: Design the feedback controller}

Feedback controllers are generally designed on the basis of a process model. As stated in the previous sections, for the LFFC, the feed-forward part is used to obtain good tracking performance. Therefore, the feedback controller should be designed such that it mainly features a robust stability. It can be done on the basis of a crude process model. The compensator that is used is of the PD-type:

$$
C(s)=K_{p}+K_{d} s
$$

The following values of the parameters are used in the simulations:

$$
K_{p}=800, K_{d}=4 .
$$

These parameters have been determined using the root locus technique.

\section{Step 2: Determine the inputs of the feed-forward part}

In the MeDe5 the plant is subject to the following disturbances: unknown mass, unknown friction force, and unknown cogging force. We illustrate Formula (3.11) 


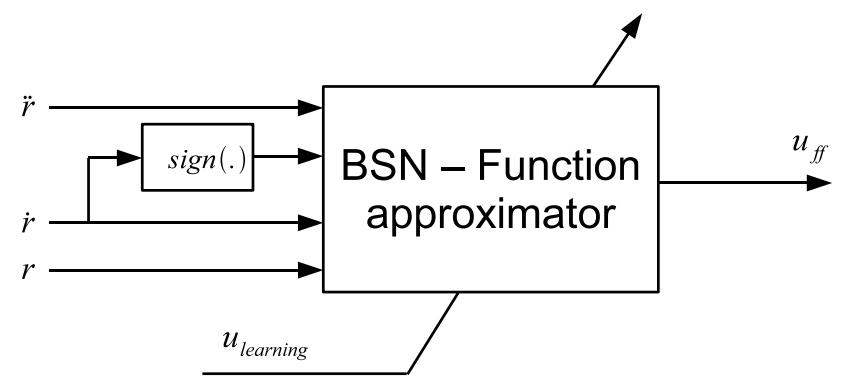

Figure 3.12: Selected inputs of the LFFC part.

for the experimental process. The state vector of the process consists of slider position $x_{2}$ and its corresponding velocity $x_{1}$. The process states for which the process output $y_{p}$ equals the desired reference input $r$ are denoted as $\dot{r}$ and $r$, giving:

$$
\begin{gathered}
x_{2}=y_{p}=r, x_{1}=\dot{r} \\
x=\left[\begin{array}{l}
x_{1} \\
x_{2}
\end{array}\right]=\left[\begin{array}{l}
\dot{r} \\
r
\end{array}\right], A=\left[\begin{array}{cc}
a_{11} & 0 \\
1 & 0
\end{array}\right]=\left[\begin{array}{cc}
-\frac{d}{m} & 0 \\
1 & 0
\end{array}\right], B=\left[\begin{array}{c}
\frac{k_{m}}{m} \\
0
\end{array}\right], \\
h(.)=\left[\begin{array}{c}
h\left(x_{1}\right)+h\left(x_{2}\right) \\
0
\end{array}\right]=\left[\begin{array}{c}
-\frac{d_{c}}{m} \operatorname{sign}(\dot{r})-\frac{c_{g}}{m} \sin (1256 r) \\
0
\end{array}\right] .
\end{gathered}
$$

The desired feed-forward signal is given by

$$
u_{d}=\frac{m}{k_{m}} \ddot{r}+\frac{d}{k_{m}} \dot{r}+\frac{d_{c}}{k_{m}} \operatorname{sign}(\dot{r})+\frac{c_{g}}{k_{m}} \sin (1256 r) .
$$

That is, we should select $[r, \dot{r}, \operatorname{sign}(\dot{r}), \ddot{r}]$ as inputs of the feed-forward controller (see Fig 3.12).

\section{Step 3: Choose the structure of the feed-forward part}

As analysed in Section 3.7, in a BSN the number of network weights that is necessary to cover the input space grows exponentially with the dimension of the inputs. Therefore, when high-dimensional BSNs are used, a large number of network weights and poor learning may result. To overcome this, splitting the multidimensional BSN into several low-dimensional BSNs that each compensate one specific disturbance, gives a solution. For the MeDe5 design, the solution to prevent the curse of dimensionality is given in Fig 3.13. The proposed network structure should consist of four BSNs that have the reference position $r$, velocity $\dot{r}$, sign of velocity, and acceleration $\ddot{r}$ as inputs. The inertia is acceleration-dependent and can be approximated by $n_{1}$ splines. The BSN that has the reference velocity $\dot{r}$ as input has to compensate for the viscous friction. To be able to do this accurately, 


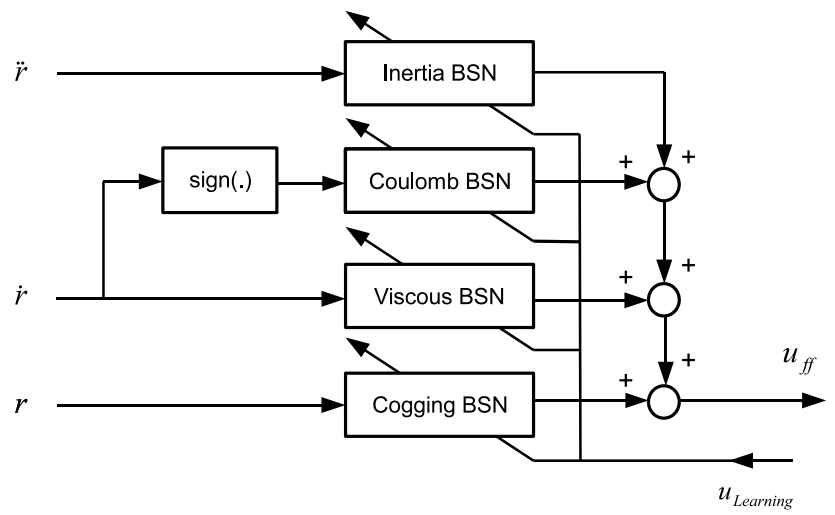

Figure 3.13: Parsimonious network structure.

we need $n_{2}$ splines. The Coulomb-friction is sign-of-velocity-dependent and can be approximated by $n_{3}$ splines. The cogging is compensated by the BSN that has the reference position $r$ as input and $n_{4}$ splines are needed. If these effects would be learnt in one four-dimensional network, the total number of weights would be equal to $n_{1} n_{2} n_{3} n_{4}$. In case all effects would be learnt in four one-dimensional BSNs, the total number of required weights would become $n_{1}+n_{2}+n_{3}+n_{4}$. The parsimonious LFFC designed for the MeDe5 is shown in Fig 3.14. Since the learning filter is not contained in the primary closed loop system, it has only limited influence on the robustness.

\section{Step 4: Choose the B-spline distribution}

Determine the minimum width of the support of the B-splines and the maximum learning rate: In order to determine the minimum width of the B-splines for which learning converges, a Bode plot of the negative complementary sensitivity function $-T$ is required. The transfer function $-T$ is determined by:

$$
-T(s)=\frac{-19\left(K_{d} s+K_{p}\right)}{s^{2}+10 s+19\left(K_{d} s+K_{p}\right)}=\frac{-76 s-15700}{s^{2}+86 s+15700}
$$

where $K_{d}$ and $K_{d}$ were given in (3.25). To obtain the minimum B-spline width, we need to determine

$$
\varphi=\arccos \left[-0.0147 \cdot \frac{|-T(j \omega)|_{\infty}}{\min _{\{\omega \in R \mid \cos (\varphi) \leq 0\}}|-T(j \omega)|}\right]
$$

From the Bode plot presented in Fig (3.15-right) it can be seen

$$
|-T(j \omega)|_{\infty}=1.71[4.65 d B]
$$




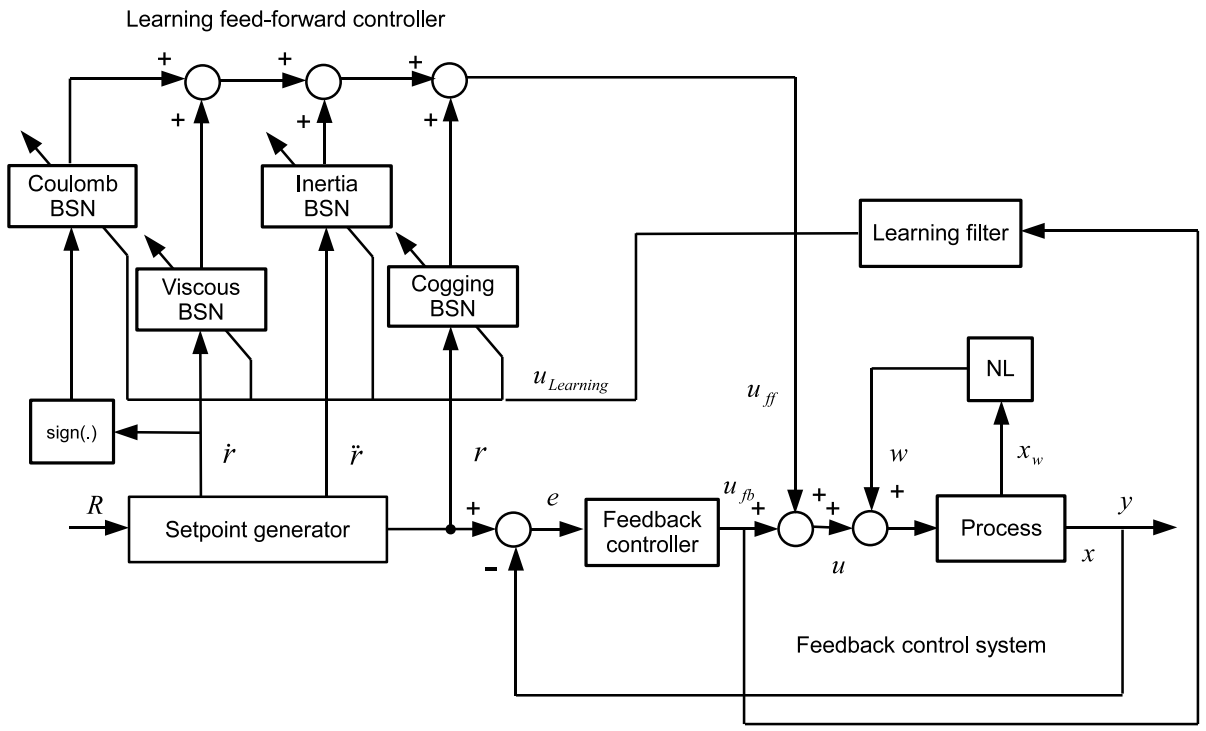

Figure 3.14: Parsimonious network structure for the MeDe5.

$$
\min _{\{\omega \in R \mid \cos (\varphi) \leq 0\}}|-T(j \omega)|=1[0 d B]
$$

This means that (3.29) results in

$$
\varphi=78.3[\mathrm{deg}]
$$

The frequency at which the phase shift is equal to 78.3 [deg] is

$$
\omega=316\left[\mathrm{rads}^{-1}\right]
$$

The minimum support of the B-splines is thus

$$
d_{\text {min }}=\frac{2 \pi}{\omega}=0.002[s]
$$

This (limit in time) will be translated to a measure in the appropriate unit later. Next, the maximum learning rate can be determined according to (3.14)

$$
\gamma \leq \frac{2}{|-T(j \omega)|_{\infty}}
$$

Using (3.30) this gives

$$
\gamma \leq \frac{2}{|-T(j \omega)|_{\infty}}=\frac{2}{1.71}=1.16
$$



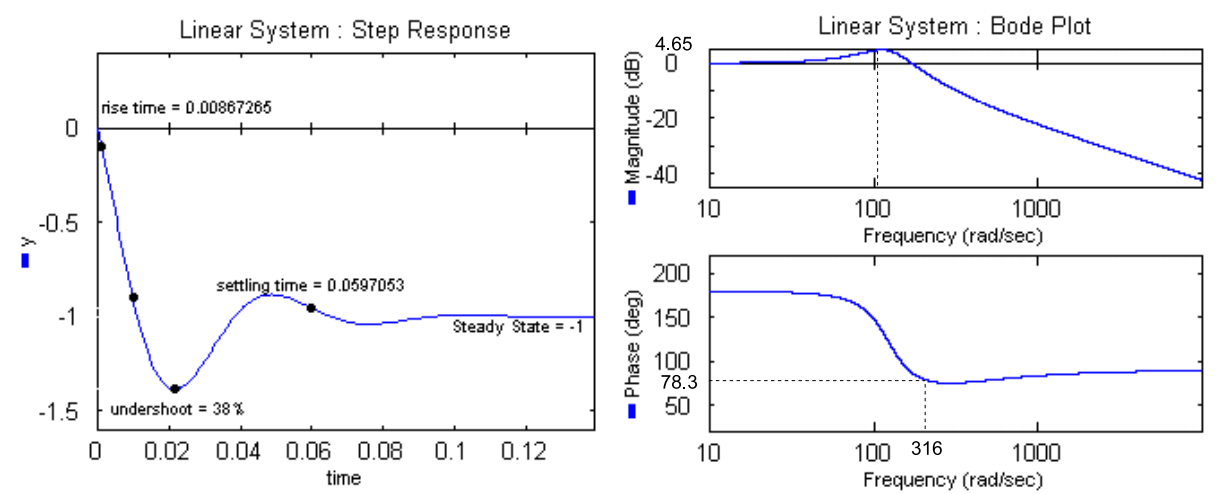

Figure 3.15: Step response of -T (left); Bode plot of -T (right).
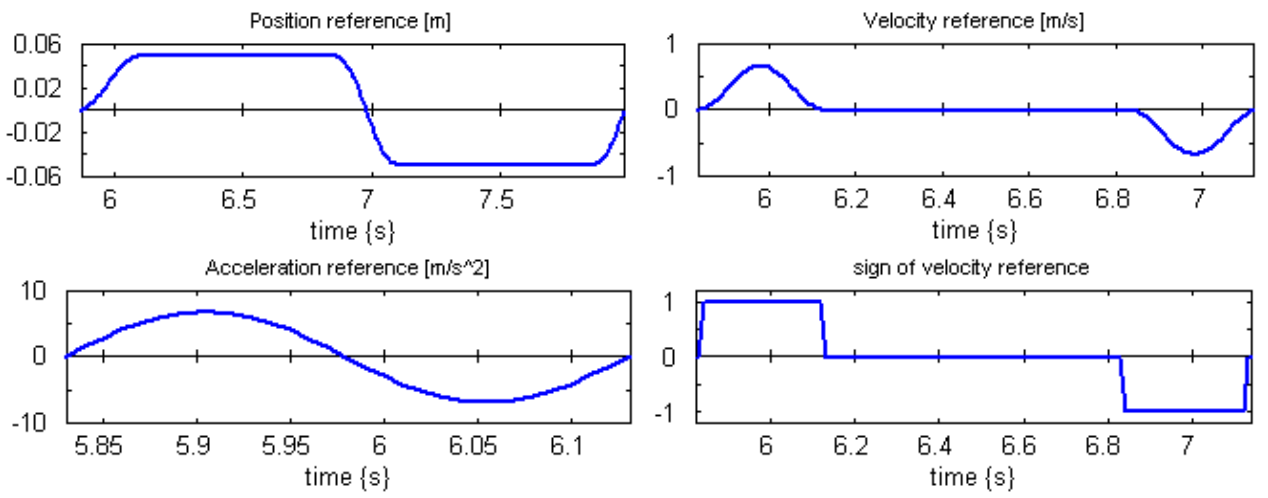

Figure 3.16: Reference inputs of the LFFC part.

- Cogging BSN: The cogging force is compensated by the BSN that has the reference position as input. The cogging force has a magnitude of $c_{g}=0.2[N]$ and has a period of $0.005[\mathrm{~m}]$. It was modeled as:

$$
F_{C}(x)=0.2 \sin \left(\frac{2 \pi}{0.005} x\right)=0.2 \sin (1256 x)[N] \text {. }
$$

To accurately compensate cogging, $25 \mathrm{~B}$-splines are defined on one cogging period. For a position range of $0.1 \mathrm{~m}$, this gives $25(0.1 / 0.005)=500 \mathrm{~B}$-splines on the input domain, $(-0.05[\mathrm{~m}], 0.05[\mathrm{~m}])$. The width of the $\mathbf{B}$-splines is $0.0042[\mathrm{~m}]$. A part of the B-spline distribution is shown in Fig 3.17. According to (3.34) the Bsplines are at least $0.002[s]$ wide, this $\mathrm{BSN}$ must be trained at a reference velocity that satisfies:

$$
\dot{r}(t)<\frac{0.0042[\mathrm{~m}]}{0.002[\mathrm{~s}]}=2.1\left[\mathrm{~ms}^{-1}\right] .
$$


- Viscous friction BSN: The BSN that has the reference velocity as input has to

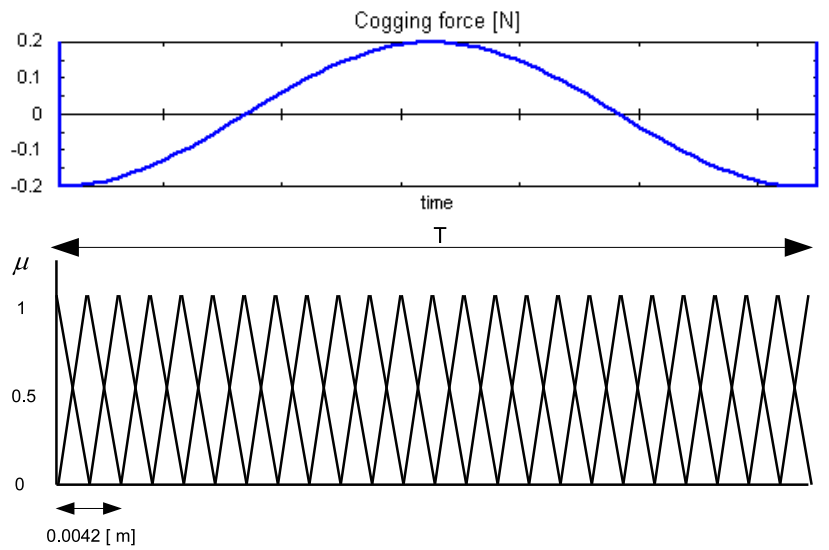

Figure 3.17: B-spline distribution of the cogging BSN.

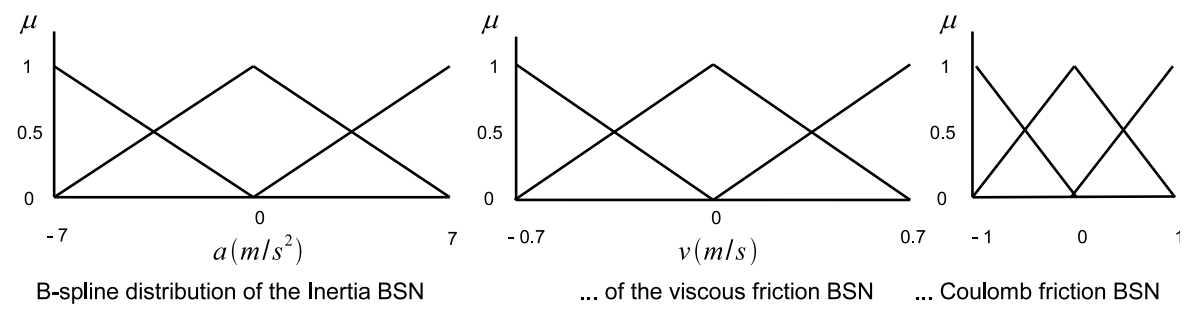

Figure 3.18: B-spline distribution ....

compensate the viscous friction. This is also a linear phenomenon. In total three B-splines have been defined on the velocity domain $\left[-0.7 \mathrm{~ms}^{-1}, 0.7 \mathrm{~ms}^{-1}\right]$. The width of the B-spline is $1.4\left[\mathrm{~ms}^{-1}\right]$. Therefore, this BSN must be trained at a reference acceleration that satisfies the following condition:

$$
\ddot{r}(t)<\frac{1.4\left[\mathrm{~ms}^{-1}\right]}{0.002[\mathrm{~s}]}=70\left[\mathrm{~ms}^{-2}\right] .
$$

- Coulomb friction BSN: The Coulomb friction is compensated by the BSN that has the reference sign of velocity as input. In total three B-splines have been defined on the input domain $[-1,1]$ (see Fig 3.18).

- Inertia BSN: The inertia is compensated by the BSN that has the reference acceleration as input. Since this is a linear phenomenon only few B-splines are needed. Therefore three B-splines are defined on the acceleration domain $\left(-7\left[\mathrm{~ms}^{-2}\right], 7\left[\mathrm{~ms}^{-2}\right]\right)$. 


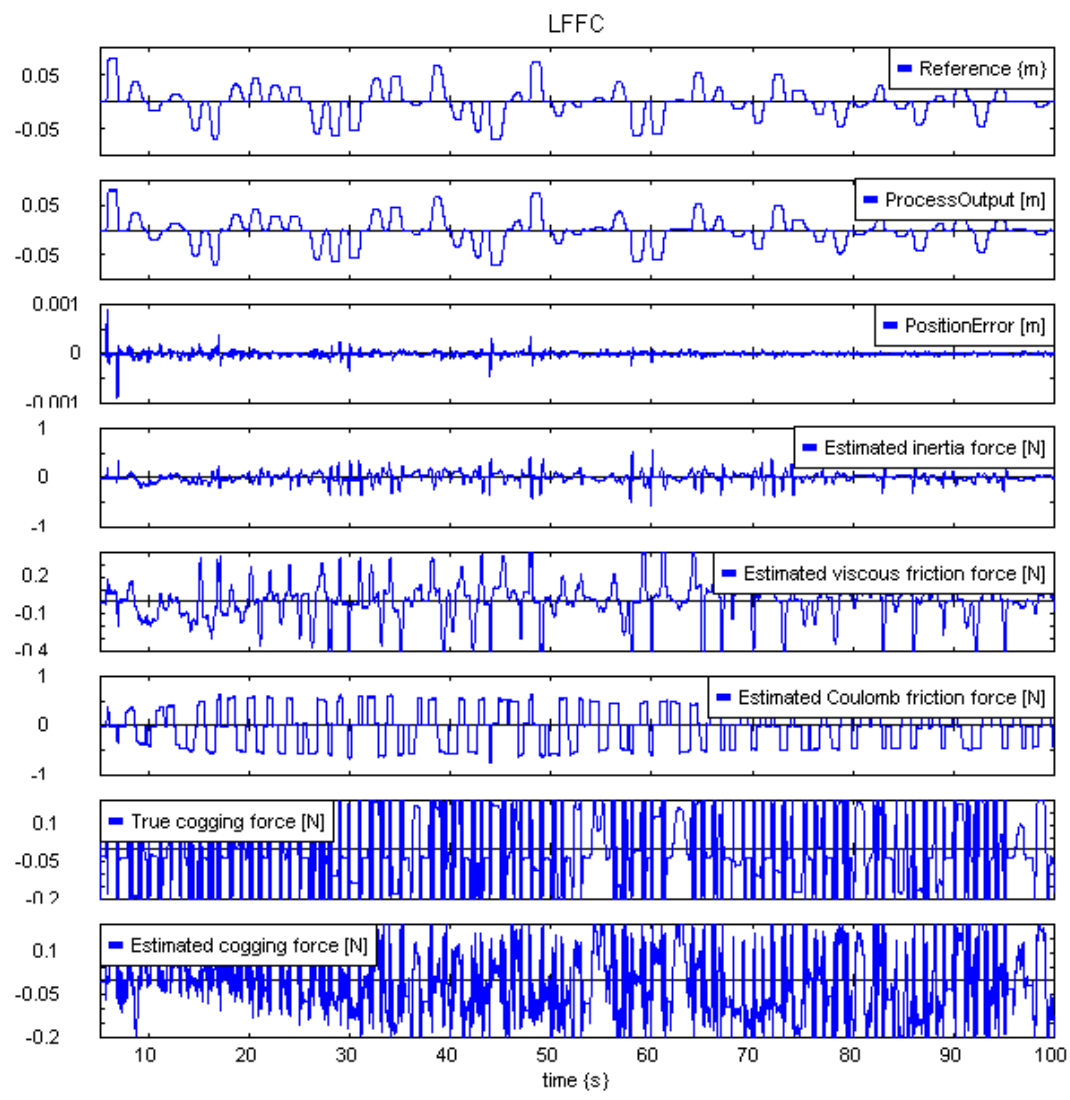

Figure 3.19: Simulation results.

\section{Step 5: Choose the learning rate}

The learning rate determines how fast the weights of the BSN are adjusted. The larger the learning rate the stronger weights can be updated, while a small learning rate implies that the weights are updated slowly. The learning rate is chosen relatively small, for example $\gamma=0.1$. Also, a large $\gamma$ means less attenuation of noise.

As can be seen in Fig 3.14, for the inertia, viscous, Coulomb, and cogging BSN in the feed-forward part the output signal from the learning filter is used to adapt the weights. The design of this filter follows from (3.21), (3.22), (3.23), (3.24), and (3.25). For (3.24) the parameters are chosen as: $\varsigma=1$, and $\omega_{n}=500[\mathrm{rad} / \mathrm{s}]$, then

$$
F(s)=\frac{s^{2}+86 s+15700}{0.000304 s^{3}+0.3668 s^{2}+139,3 s+15700}
$$


To demonstrate the usefulness and accuracy of the method, numerical simulations will be performed in which the plant is controlled by a parsimonious LFFC. The plant has to track random reference motions. The following observations are given:

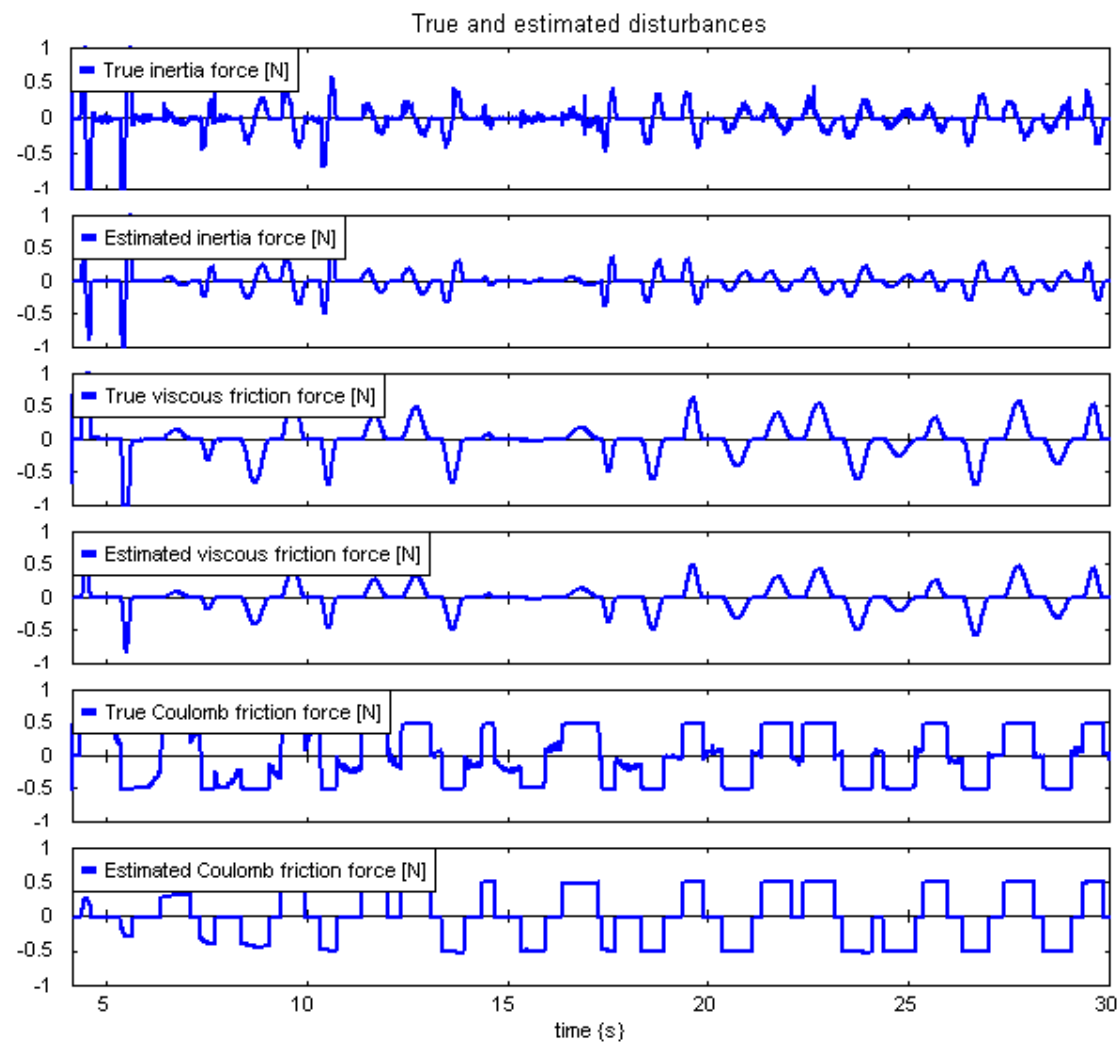

Figure 3.20: True disturbances in the model and corresponding estimated disturbances.

1- The parameters of the feedback controller are calculated in such way that it does not need to get a high performance. However, it guarantees robust stability. Hence, when the feedback controller is used alone the tracking error is large (see Fig 3.22a).

2- When the linear compensators (inertia and viscous friction) in the feedforward part are added, after 20 [s] on-line operations the tracking error is visibly decreased. By comparing two simulation results as indicated in Fig 3.22a and Fig 3.22b, we conclude that the parsimonious LFFC is able to compensate for the unknown inertia and unknown viscous friction.

3- The simulation results of the system in cases without and with Coulomb friction compensation are given in Fig 3.22. The contribution of the Coulomb 
friction compensator is clearly shown. In Fig 3.22c, without Coulomb friction compensator, the effect of Coulomb friction will result in a large tracking error. However, when the Coulomb friction BSN is added, this effect is considerably compensated (see Fig 3.22d). After a few motions the tracking error will converge to a smaller value. It is observed that the Coulomb friction force is significantly compensated in the feed-forward part (also indicated in Fig 3.20).

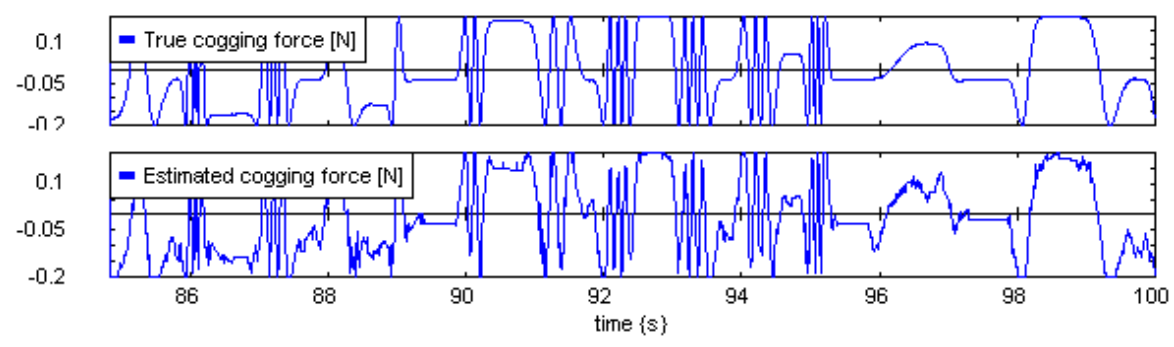

Figure 3.21: True and estimated cogging force.

4- The role of the cogging compensator is clearly shown by comparing the simulation results as indicated in Fig 3.22e and Fig 3.22f. When the cogging BSN is used, the tracking error is considerably decreased. In other words, the unknown cogging force is well compensated in the feed-forward controller (also indicated in Fig 3.21).
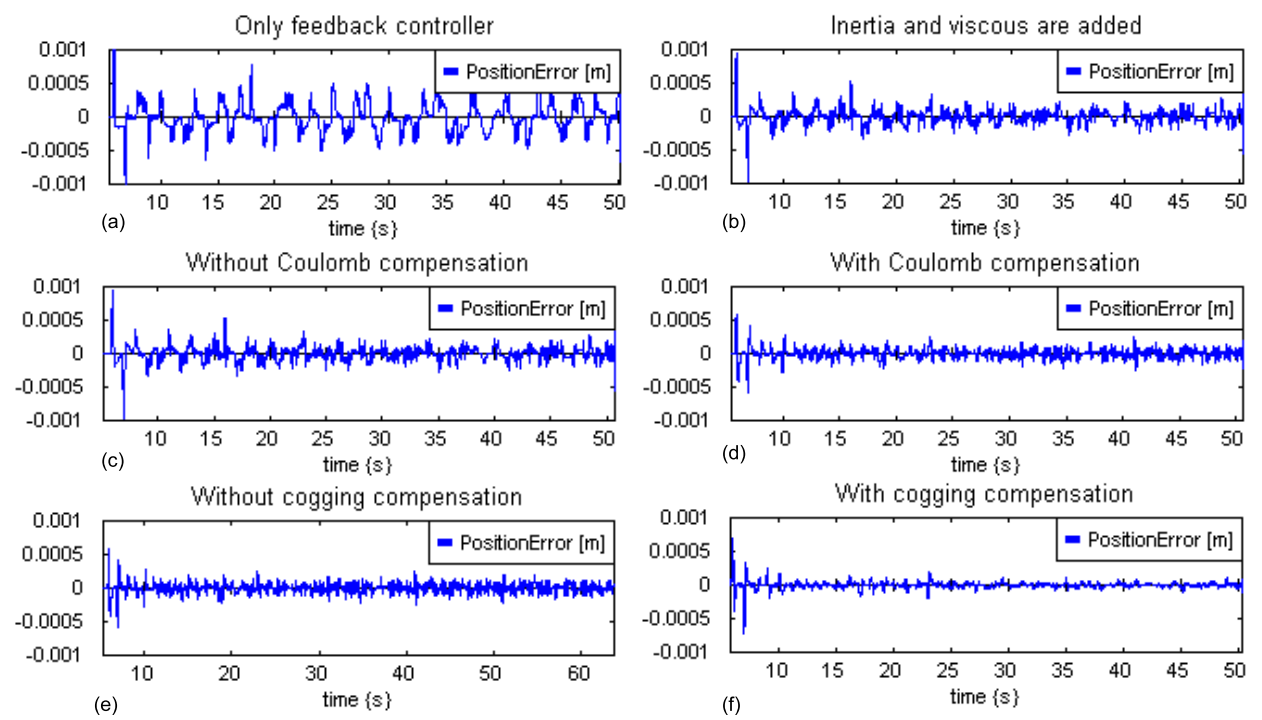

Figure 3.22: Comparison of simulation results. 
From the obtained observations we can conclude that the LFFC is suitable for controlling motion systems that are subject to reproducible disturbances. LFFC offers several potential advantages over conventional control algorithms. The effectiveness of the considered control strategy was verified through the simulation results. The LFFC was well able to compensate for the known disturbances. A fast rate of convergence and high robustness can be obtained. After a short training time, the parsimonious LFFC was able to obtain about a 7 times smaller peaks in the tracking error than the feedback controller (compare 3.22a with 3.22f).

\subsection{Conclusions}

Since motion systems may have complicated nonlinear mathematical models, control algorithms that require accurate process models are difficult to implement. To overcome this, several learning control methods have been proposed. These controllers are not designed fully prior to operation but trained during control. The characteristics of the Learning Control introduced here are that the controller can be chosen as the inverse dynamical model of the plant and it is composed of the feed-forward loop besides the feedback controller loop. This type of learning control is known as the learning feed-forward control scheme. The feedback controller can be designed for robustness mainly, which does not require an accurate process model. The feedback controller compensates unknown disturbances and it does not have to know exactly how a system will respond to disturbances. The feed-forward controller compensates the reproducible disturbances that depend on the state of the process before they can affect the system. Normally, a feed-forward controller is designed on the basis of an accurate process model of the process. The obtained LFFC does not suffer from a trade-off between high performance and robust stability.

In LFFC, a B-spline network is chosen for the feed-forward part. In order to learn the input-output behavior of the inverse dynamical model of the controlled object, a learning signal is required. The output of the feedback controller provides the training signal for the LFFC. The feed-forward design is formulated as a linear program that optimizes the number and position of the knots of the splines. Detailed design formulas were given for two parameters of B-spline network: the B-spline support width and the learning gain are given based on stability analysis in frequency domain [45]. There is a tradeoff between accuracy and computational complexity of training, since the number of inputs used affects the number of weights in the neural network. An on-line B-spline neural network is used because of its local weight-updating characteristic, which has the advantages of fast convergence speed and low computation complexity. This is very important for real-time control applications. 
The inputs of the feed-forward part depend on the plant and the reproducible disturbances that the LFFC has to compensate. In case of random reference motions the reference signal and its derivatives/integrals, are used as inputs. A correct state space representation of the plant is used to determine the specific inputs. Each of reference inputs of the LFFC will be used to compensate one specific disturbance.

The width of the B-splines should be chosen such that the BSN is able to learn the desired feed-forward signal. The smaller the support of the B-splines the more accurate the LFFC can be obtained. However, a too small support may result in unstable behavior. The frequency response of the closed loop system can be used to determine the minimum width of the B-splines, for which learning converges. The B-spline distribution is then chosen on the basis of qualitative process knowledge.

When the inputs of the BSN are chosen straightforwardly on basis of the plant model, a multidimensional BSN results. Large network sizes and poor generalizing ability may result, known as the curse of dimensionality. A parsimonious LFFC offers a solution by splitting up the multidimensional BSN into several lower-dimensional BSNs. In this case the implementation requires a low number of weights, which means that it is memory efficient and learn relatively fast, while it is relatively insensitive to noise. The parsimonious network structure can be designed on the basis of qualitative knowledge of the process and disturbances.

The effectiveness of the LFFC is illustrated through comparison with a PID controller, obtained by observing the system dynamic performances. The simulation results confirm that the LFFC is an attractive control strategy for motion systems that are subject to reproducible disturbances. 


\section{Chapter 4}

\section{LQG combined with MRAS-based LFFC}

\subsection{Introduction}

In Chapter 3, a function approximator with "standard" neural networks was used which enabled to approximate complex non-linear functions [41, 45]. However, they are not always suited for controller design applications because of too many adjustable parameters. Moreover, using neural networks for simple tasks is unnecessary. To deal with this problem, a MRAS-based learning feed-forward controller has been applied [43]. The idea of LFFC is applied but without using the complex B-spline neural networks. Instead, we propose to use MRAS-based adaptive components.

In this chapter we introduce a control structure, which consists of an LQG controller and an MRAS-based learning feed-forward controller. Feed-forward is used in addition to LQG, because LQG is still required to account for imperfections in the feed-forward system model. By adding a (learning) feed-forward component to the LQG controller, an extra degree of freedom in the controller design is created. A control scheme is derived which has good properties in common with both the LQG and LFFC controller. This control structure can capture many aspects of the control problem as mentioned in Chapter 1 . The responses to setpoint changes and reproducible disturbances are mainly obtained by the LFFC. The LQG is designed to obtain a stable closed-loop system that is insensitive to measurement noise and variations and uncertainties in process behavior.

This chapter is organized as follows. In Section 4.2, the new LFFC is introduced. It is called an MRAS-based Learning Feed-forward Control. The LFFC is used to cancel the unwanted signal, disturbing the input of the plant. Theoretical background of the MRAS-based LFFC is also given in this section. The design 
of the proposed controller is introduced in Section 4.3. The controller structures with Coulomb friction force and Cogging force compensation are given in Sections 4.3.3 and 4.3.4, respectively. At the end of this chapter, a review of the chapter is given, including a comparison with previous methods.

\subsection{An MRAS-based Learning Feed-Forward Con- troller}

This section will deal with the basic theory of an MRAS-based learning feedforward control as presented in the paper of Van Amerongen [43]. As was introduced in Chapter 3, LFFC consists of a feedback and a feed-forward controller. The feed-forward part is considered as a function approximator whose input-output mapping can be adapted during control and is intended to become the (stable part of the) inverse of the plant $[13,45]$. It is clear that if an accurate model of the process is available, and if its inverse exists, then process dynamics can be canceled by the inverse model. As a result, the output of the process will be equal to the desired output if no other disturbances are present. In order to approximate the state dependent function, some kind of function approximator is introduced. In this study, in order to obtain high stability and fast convergence for the design of a linear process, the feed-forward part is proposed using adaptive components. The mechanism that adjusts the input-output mapping of the adaptive components is based on the tracking error. The well-known Liapunov approach is used to find stable adaptive laws for the feed-forward parameters in such way that learning converges.

\subsubsection{Theoretical Background}

\subsubsection{Introduction}

As was introduced in Chapter 2, there are two main types of MRAS ([40, 4] and [22]). 1. Direct MRAS : the idea is to create a closed loop controller with parameters that can be adjusted to change the response of the system. The output of the process is compared to a desired response from a reference model. The controller parameters are adjusted based on this error. Finally, the controller parameters converge to suitable values, that is, the process response tracks the reference response with a vanishing error. 2. Indirect MRAS: there are two tasks at each time step. The first is the identification of relevant plant parameters. Then, the controller parameters are computed based on the estimated plant parameters.

A setpoint generator, in mechatronic systems called a motion profile, can also act and be used as a reference model. This leads to the basic structure of Fig 


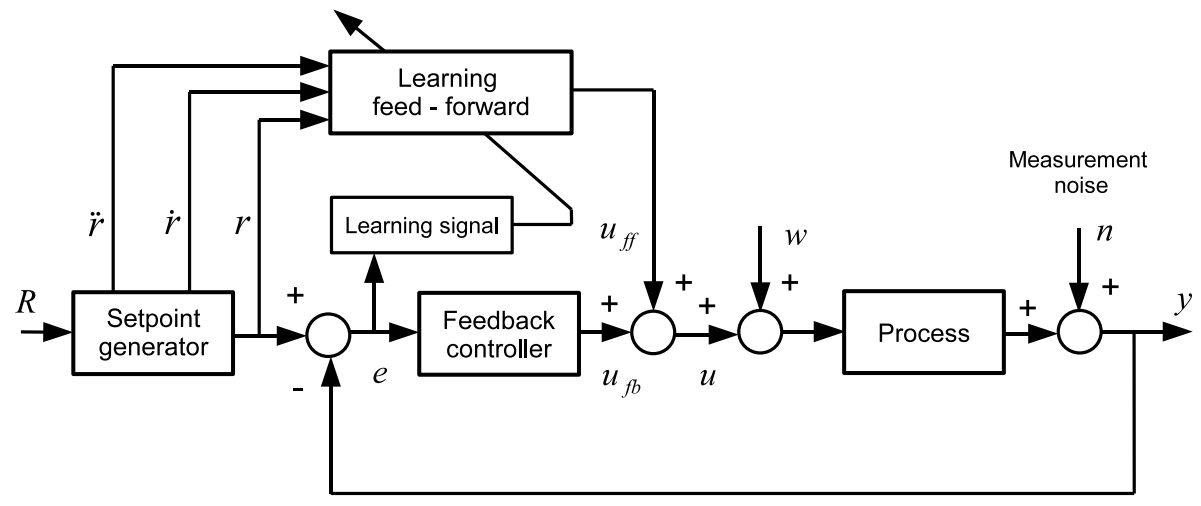

Figure 4.1: Learning Feed-Forward Controller

4.1. A typical controller for a high-precision motion system consists of a feedforward controller and a feedback controller. The inputs to the feed-forward part are the states of the setpoint generator. The feed-forward controller generates a feed-forward signal by summing the profile setpoint signals with properly chosen weights. The feed-forward parameters are adjusted all the time. This implies that they follow changes in the process. As a result, it can be expected that a proper feed-forward controller signal is generated, effective for providing good tracking control performance. Note that, addition of the proper feed-forward component may improve performance, without affecting the stability, and robustness properties [5].

\subsubsection{MRAS-based Feed-Forward Control}

A state variable filter (SVF) can be used as the reference model to generate a set of profile setpoint signals including position, velocity, and acceleration. For the second-order case, such a filter is described by a second order transfer function. Note that, denominator coefficients should always have positive values to guarantee stabilization. By means of the feed-forward controller, the SVF output signals can be used to generate an inverse model of the process. Let the feedback controlled process be described by the transfer function $H_{p}$, and the reference model by $H_{r e f}$

$$
\begin{gathered}
H_{p}=\frac{1}{a_{p} s^{2}+b_{p} s+c_{p}} \\
H_{r e f}=\frac{\omega_{n}^{2}}{s^{2}+2 \varsigma \omega_{n} s+\omega_{n}^{2}}
\end{gathered}
$$




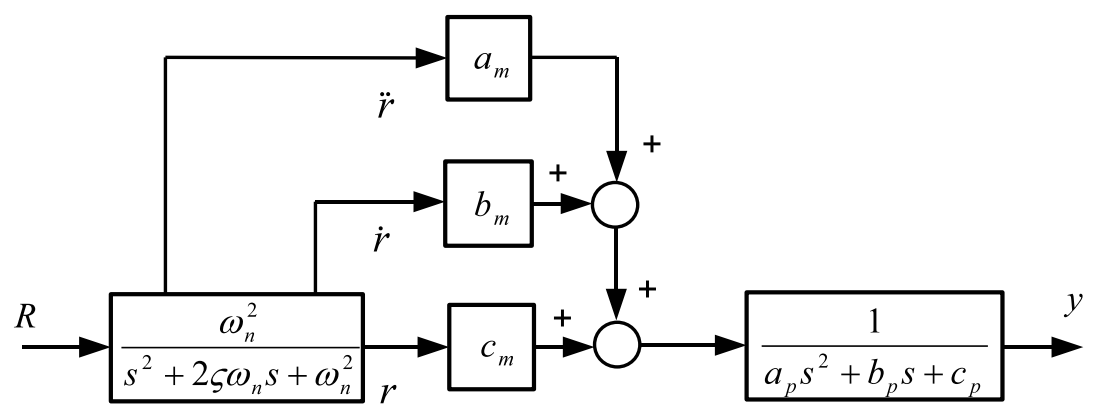

Figure 4.2: Realization of an inverse model of the process

In Figure 4.2 the transfer from reference $R$ to process output $Y$ is:

$$
H_{t o t}=\frac{\omega_{n}^{2}}{s^{2}+2 \varsigma \omega_{n} s+\omega_{n}^{2}} \frac{a_{m} s^{2}+b_{m} s+c_{m}}{a_{p} s^{2}+b_{p} s+c_{p}}
$$

If $a_{m}, b_{m}$ and $c_{m}$ are equal to respectively $a_{p}, b_{p}$ and $c_{p}, H_{t o t}$ is equal to the desired response, given by $H_{\text {ref }}$. The desired response from the setpoint generator is compared to the process response. Based on this error, we should try to find a learning mechanism that adjusts the parameters $a_{m}, b_{m}$ and $c_{m}$, such that they converge to $a_{p}, b_{p}$ and $c_{p}$, respectively. When there are disturbances present, the first thing that might come to mind is using a feedback controller. However, when we model these disturbances as a 'constant' disturbance, this disturbance could also be compensated in a feed-forward manner by adding a 'constant' input $d_{m}$ to be found by the learning mechanism. The resulting controller is given in Fig 4.3.

\subsubsection{Adaptive laws}

As can be seen in Fig 4.3, the setpoint generator can be used as the reference model. The learning signal is determined by the difference between the output of the reference model and the process. In order to find adaptive laws for the feedforward parameters $a_{m}, b_{m}, c_{m}$ and $d_{m}$, such that they converge to ideal values that cause the process response matches the response of the reference model, the Liapunov stability approach can be used. The design procedure will be illustrated with the second-order example. We assume that the process parameters are unknown and vary slowly. In general, the feedback controller is used to compensate for random disturbances and possibly also to generate a learning signal for the learning mechanism. We assume at this stage that the disturbance is zero. The design problem is thus: Find (stable) adjustment laws for the adjustable parameters $a_{m}, b_{m}$, and $c_{m}$ such that the error e between the reference model and the process as well as the errors in the feed-forward parameters asymptotically go to zero. 


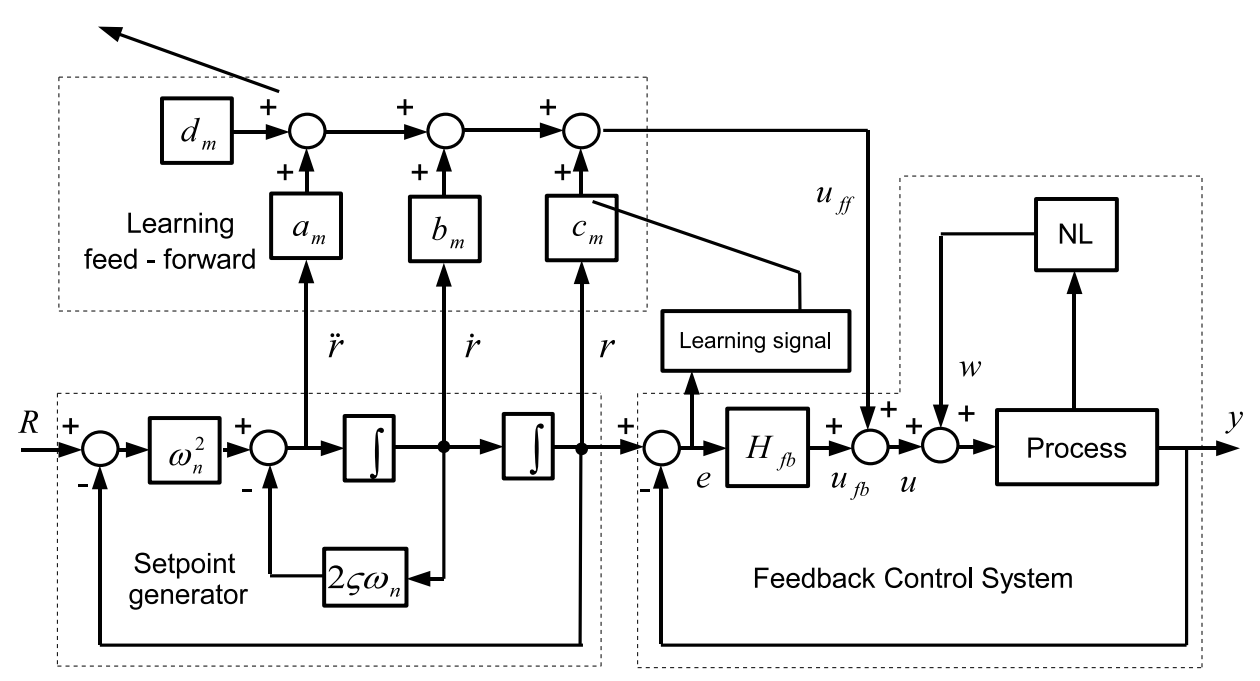

Figure 4.3: MRAS-based Learning Feed-Forward Controller

Equations (4.1) and (4.2) are rewritten in state space form. This gives the second-order reference model:

$$
\dot{x}_{m}=\left[\begin{array}{cc}
0 & 1 \\
-\omega_{n}^{2} & -2 \varsigma \omega_{n}
\end{array}\right] x_{m}+\left[\begin{array}{c}
0 \\
\omega_{n}^{2}
\end{array}\right] R
$$

An extra state $x_{m, 3}$ is defined as

$$
x_{m, 3}=\dot{x}_{m, 2}=\omega_{n}^{2} R-\omega_{n}^{2} x_{m, 1}-2 \varsigma \omega_{n} x_{m, 2}
$$

This gives the process equations

$$
\dot{x}_{p}=\left[\begin{array}{cc}
0 & 1 \\
-\frac{c_{p}}{a_{p}} & -\frac{b_{p}}{a_{p}}
\end{array}\right] x_{p}+\left[\begin{array}{cc}
0 & 0 \\
\frac{c_{m}}{a_{p}} & \frac{b_{m}}{a_{p}}
\end{array}\right] x_{m}+\left[\begin{array}{c}
0 \\
\frac{a_{m}}{a_{p}}
\end{array}\right] x_{m, 3} .
$$

Because the parameters $a_{m}, b_{m}$, and $c_{m}$ should converge to $a_{p}, b_{p}$ and $c_{p}$ we define the error in the parameters as

$$
\begin{gathered}
\frac{a_{m}-a_{p}}{a_{p}}=\frac{a_{m}}{a_{p}}-1=a \Rightarrow \frac{a_{m}}{a_{p}}=1+a \\
\frac{b_{m}-b_{p}}{a_{p}}=b_{m}^{\prime}-b_{p}^{\prime}=b \\
\frac{c_{m}-c_{p}}{a_{p}}=c_{m}^{\prime}-c_{p}^{\prime}=c .
\end{gathered}
$$


After substitution of equations (4.7) in equation (4.6), and using (4.5) the process model is modified as

$$
\begin{aligned}
\dot{x}_{p}= & {\left[\begin{array}{cc}
0 & 1 \\
-c_{p}^{\prime} & -b_{p}^{\prime}
\end{array}\right] x_{p}+\left[\begin{array}{cc}
0 & 0 \\
c_{m}^{\prime} & b_{m}^{\prime}
\end{array}\right] x_{m}+} \\
& +\left[\begin{array}{cc}
0 & 0 \\
-\omega_{n}^{2} & -2 \varsigma \omega_{n}
\end{array}\right] x_{m}+\left[\begin{array}{c}
0 \\
\omega_{n}^{2}
\end{array}\right] R+\left[\begin{array}{l}
0 \\
a
\end{array}\right] x_{m, 3}
\end{aligned}
$$

We define the error $e=\left(e_{1}, e_{2}\right)^{T}=x_{m}-x_{p}$. By subtracting equation (4.8) from equation (4.4), it follows that

$$
\begin{aligned}
\dot{e}= & {\left[\begin{array}{cc}
0 & 1 \\
-\omega_{n}^{2} & -2 \varsigma \omega_{n}
\end{array}\right] x_{m}+\left[\begin{array}{c}
0 \\
\omega_{n}^{2}
\end{array}\right] R-\left[\begin{array}{cc}
0 & 1 \\
-\omega_{n}^{2} & -2 \varsigma \omega_{n}
\end{array}\right] x_{m}-} \\
& -\left[\begin{array}{c}
0 \\
\omega_{n}^{2}
\end{array}\right] R-\left[\begin{array}{cc}
0 & 1 \\
-c_{p}^{\prime} & b_{p}^{\prime}
\end{array}\right] x_{p}-\left[\begin{array}{cc}
0 & 0 \\
c_{m}^{\prime} & b_{m}^{\prime}
\end{array}\right] x_{m}-\left[\begin{array}{l}
0 \\
a
\end{array}\right] x_{m, 3}
\end{aligned}
$$

This simplifies into:

$$
\dot{e}=\left[\begin{array}{cc}
0 & 1 \\
-c_{m}^{\prime} & -b_{m}^{\prime}
\end{array}\right] x_{m}-\left[\begin{array}{cc}
0 & 1 \\
-c_{p}^{\prime} & -b_{p}^{\prime}
\end{array}\right] x_{p}-\left[\begin{array}{l}
0 \\
a
\end{array}\right] x_{m, 3}
$$

and can be rewritten as

$$
\dot{e}=A_{m} x_{m}-A_{p} x_{p}-\left[\begin{array}{l}
0 \\
a
\end{array}\right] x_{m, 3}
$$

or

$$
\begin{gathered}
\dot{e}=A_{m} x_{m}-\underbrace{A_{p} x_{m}+A_{p} x_{m}}_{=0}-A_{p} x_{p}-\left[\begin{array}{l}
0 \\
a
\end{array}\right] x_{m, 3} \\
=\underbrace{A_{m} x_{m}-A_{p} x_{m}}_{A^{\prime} x_{m}}+\underbrace{A_{p} x_{m}-A_{p} x_{p}}_{A_{p} e}-\left[\begin{array}{l}
0 \\
a
\end{array}\right] x_{m, 3} \\
= \\
A^{\prime} x_{m}-A_{p} e-\left[\begin{array}{l}
0 \\
a
\end{array}\right] x_{m, 3},
\end{gathered}
$$

in which the matrix $A^{\prime}$ contains the parameter errors $b$ and $c$

$$
A^{\prime}=A_{m}-A_{p}=\left[\begin{array}{cc}
0 & 0 \\
-c & -b
\end{array}\right]
$$

Combining the parameter errors $a, b$ and $c$ in the error vector $\varepsilon$ and in the error matrix $A$ and redefining $x_{m}$ gives

$$
\varepsilon=\left[\begin{array}{l}
-c \\
-b \\
-a
\end{array}\right], A=\left[\begin{array}{ccc}
0 & 0 & 0 \\
-c & -b & -a
\end{array}\right], x_{m}=\left[\begin{array}{c}
x_{m, 1} \\
x_{m, 2} \\
x_{m, 3}
\end{array}\right], \dot{e}=A x_{m}+A_{p} e .
$$


In order to proof the asymptotic stability of $e$ we define a positive definite Liapunov function:

$$
V=e^{T} P e+\varepsilon^{T} \alpha \varepsilon,
$$

in which $P$ a positive definite symmetrical matrix and $\alpha$ a diagonal matrix with in principle arbitrary coefficients $>0$. For a stable adaptive system the time derivative of $V$ should be $\leq 0$ :

$$
\frac{d V}{d t}=\dot{e}^{T} P e+e^{T} P \dot{e}+\dot{\varepsilon}^{T} \alpha \varepsilon+\varepsilon^{T} \alpha \dot{\varepsilon} .
$$

Substituting $\dot{e}$ from (4.14) and rearranging the terms yields:

$$
\frac{d V}{d t}=e^{T} A_{p}^{T} P e+e^{T} P A_{p} e+2 e^{T} P A x_{m}+2 \varepsilon^{T} \alpha \dot{\varepsilon} .
$$

With the first two terms of (4.17) equal to:

$$
e^{T}\left(A_{p}^{T} P+P A_{p}\right) e=-e^{T} Q e
$$

It follows that:

$$
\frac{d V}{d t}=-e^{T} Q e+2 e^{T} P A x_{m}+2 \varepsilon^{T} \alpha \dot{\varepsilon}
$$

When $Q$ is a positive definite symmetrical matrix the first term in equation (4.19) is always negative or zero and when the other terms are made equal to zero, $\frac{d V}{d t} \leq 0$. When $A_{p}$ is stable, we can find for any positive definite symmetrical matrix $Q$ a positive definite symmetrical matrix $P$ by solving the Liapunov equation

$$
A_{p}^{T} P+P A_{p}=-Q .
$$

If the process matrix $A_{p}$ is not stable, the process must be stabilized first by appropriate feedback.

The adjustment rules for the feed-forward gains follow from the condition that the remaining terms should be zero.

$$
2 e^{T} P A x_{m}+2 \varepsilon^{T} \alpha \dot{\varepsilon}=0 \Leftrightarrow \varepsilon^{T} \alpha \dot{\varepsilon}=-e^{T} P A x_{m} .
$$

After simplification (taking into account that only the last row of $A$ contains values $\neq 0$ ) this yields:

$$
\frac{d \varepsilon}{d t}=-\alpha^{-1}\left(p_{21} e+p_{22} \dot{e}\right) x_{m} .
$$

If it is assumed that the adaptation is fast compared with the variations in the process parameters it follows that:

$$
\frac{d \varepsilon}{d t}=\frac{d}{d t}\left[\begin{array}{c}
-\left(c_{m}+c_{p}\right) / a_{p} \\
-\left(b_{m}+b_{p}\right) / b_{p} \\
-\left(a_{m} / a_{p}-1\right)
\end{array}\right]=-\frac{d}{d t}\left[\begin{array}{c}
c_{m} / a_{p} \\
b_{m} / a_{p} \\
a_{m} / a_{p}
\end{array}\right]
$$


When the unknown "constant" parameter $a_{p}$ is part of the ('arbitrary') adaptive gains $\alpha^{-1}$, this yields the following adjustment rules:

$$
\begin{aligned}
a_{m} & =\frac{1}{\alpha_{33}} \int\left[\left(p_{21} e+p_{22} \dot{e}\right) x_{m, 3}\right] d t+a_{m}(0) \\
b_{m} & =\frac{1}{\alpha_{22}} \int\left[\left(p_{21} e+p_{22} \dot{e}\right) x_{m, 2}\right] d t+b_{m}(0) \\
c_{m} & =\frac{1}{\alpha_{11}} \int\left[\left(p_{21} e+p_{22} \dot{e}\right) x_{m, 1}\right] d t+c_{m}(0)
\end{aligned}
$$

Like in any MRAS-based system, adaptive disturbance compensation can be added, by realizing that the parameter $d_{m}$ acts on an extra input signal 1, instead of on one of the state variables:

$$
d_{m}=\frac{1}{\gamma} \int\left[\left(p_{21} e+p_{22} \dot{e}\right) 1\right] d t+d_{m}(0)
$$

in which $1 / \alpha_{11}, 1 / \alpha_{22}, 1 / \alpha_{33}$, and $1 / \gamma$ are called the adaptive gains. They determine the speed of adaptation and they can, under the condition that they are larger than zero, in principle, be arbitrarily chosen. Equations (4.24) can be generalized to an equation for higher-order systems. For an $n^{t h}$-order system we find for parameter $a_{m, i}$ :

$$
\frac{d a_{m, i}}{d t}=\frac{1}{\alpha_{i i}}\left(\sum_{k=1}^{n} P_{n k} e_{k}\right) x_{m, i}
$$

In the adjustment laws the derivative of the error is needed. This derivative can be obtained by means of a second-order state variable filter. The bandwidth of this filter has to be at least 10 times larger than the bandwidth of the reference model in order not to endanger the stability of the system. On the other hand, the combination of a state variable filter with not too large bandwidth and use of the model states for the adaptation as well as for the control makes the system relatively insensitive for noisy measurements and leads to a robust system.

\subsection{Design of the proposed controller}

The obtained knowledge in the previous sections is now used to formulate a practical design procedure. Fig 4.4 shows the block diagram of the proposed control structure, which combines an MRAS-based LFFC and a separate LQG controller. In the feed-forward control part, the parameter adaptation is driven by the tracking error between reference output and measured process output, while in the LQG part the observer is driven by the prediction errors between measured process variables and corresponding estimated variables. 


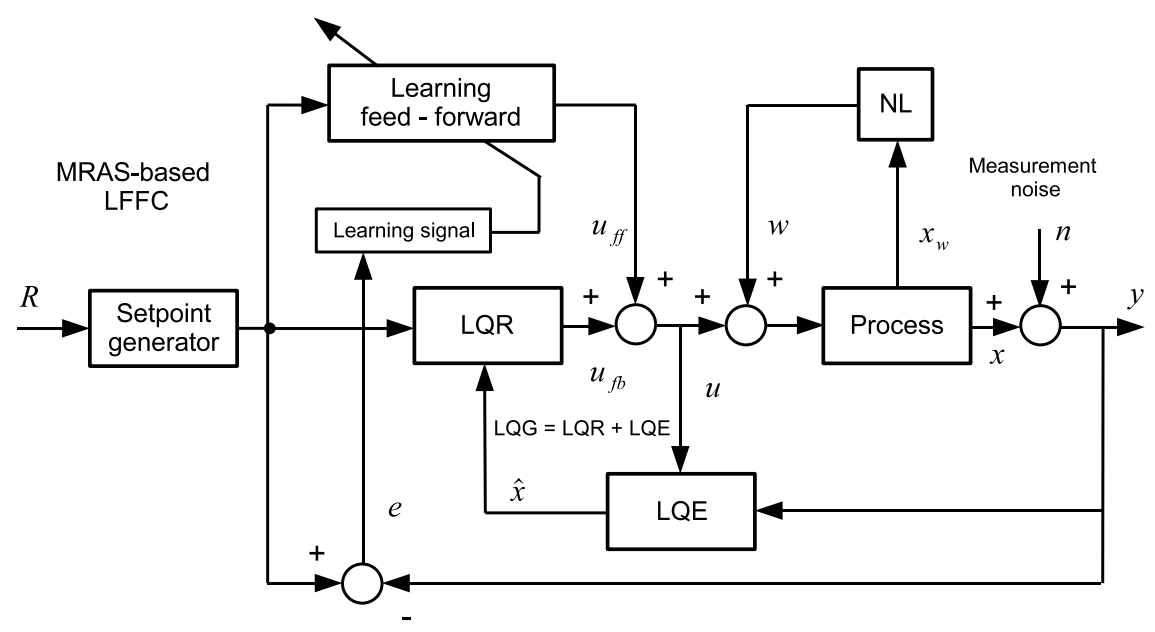

Figure 4.4: LQG combined with MRAS-based LFFC

For the MeDe5 setup, in order to take the flexible frame behavior into account, in this study the LQG design is based on the $4^{\text {th }}$ order approximation of the $6^{\text {th }}$ order model. The performance of the proposed controller will be investigated with the tracking error due to following a random trajectory. The ability to track a trajectory in the presence of load changes will be evaluated. The ability to attenuate the effect of measurement noise acting on the process output will also be investigated. By separating the design objectives, decoupling the MRAS-based LFFC, and the LQG controller, both objectives can be achieved.

There are two main design steps. In the first step, the design of an MRASbased LFFC is carried out and in the second step we address the design of an LQG controller.

\subsubsection{Design the MRAS-based LFFC}

In practice, the MeDe5 is implemented based on computer control. Therefore, the continuous-time feed-forward controller given in Section 4.3 has to be replaced by a discrete-time feed-forward controller.

- Instead of using equation

$$
\frac{d P_{c}}{d t}=A_{m}^{T} P_{c}+P_{c} A_{m}+Q_{c}
$$

where $A_{m}$ is the continuous time state matrix of the reference model, the Euler 
approximation of this equation is given

$$
\begin{aligned}
\frac{\operatorname{next}(P)-P}{T_{s}} & =A_{m}^{T} P+P A_{m}+Q_{d}, \\
\rightarrow \operatorname{next}(P) & =\left(A_{m}^{T} P+P A_{m}+Q_{d}\right) T_{s}+P,
\end{aligned}
$$

in which, $T_{s}$ is the sampling interval.

- The continuous-time integrators in Equation (4.24) are replaced by, the discrete-time integrators.

$$
\begin{aligned}
a_{m} & =\frac{1}{\alpha_{33}} \sum\left[\left(p_{21} e+p_{22} \dot{e}\right) x_{m, 3}\right] T_{s}+a_{m}(0), \\
b_{m} & =\frac{1}{\alpha_{22}} \sum\left[\left(p_{21} e+p_{22} \dot{e}\right) x_{m, 2}\right] T_{s}+b_{m}(0), \\
c_{m} & =\frac{1}{\alpha_{11}} \sum\left[\left(p_{21} e+p_{22} \dot{e}\right) x_{m, 1}\right] T_{s}+c_{m}(0) .
\end{aligned}
$$

- The continuous-time reference model and state variable filter in the learning block solve the following differential equation

$$
\frac{d x}{d t}=A_{F} x+B_{F} u
$$

In discrete time domain, this could be approximated with the Euler approximation given by:

$$
\begin{aligned}
\frac{\operatorname{next}(x)-x}{T_{s}} & =A_{F} x+B_{F} u \\
\rightarrow \operatorname{next}(x) & =\left(A_{F} x+B_{F} u\right) T_{s}+x .
\end{aligned}
$$

Feedback controller: As discussed in the previous sections, tracking performance is obtained by the MRAS-based LFF. The feedback controller is designed such that it features robust stability for closed loop when used alone. Initially, the feedback controller is of the PD-type with the chosen parameters $K_{p}=100$ and $K_{d}=1$.

Reference model: Explicit position, velocity, and acceleration profile setpoint signals are created using the reference model, which is described by the transfer function

$$
H_{r e f}=\frac{\omega_{n}^{2}}{s^{2}+2 \varsigma \omega_{n} s+\omega_{n}^{2}} .
$$

The parameters of the reference model are chosen such that the higher order dynamics of the system will not be activated [22]. The following values are chosen: 


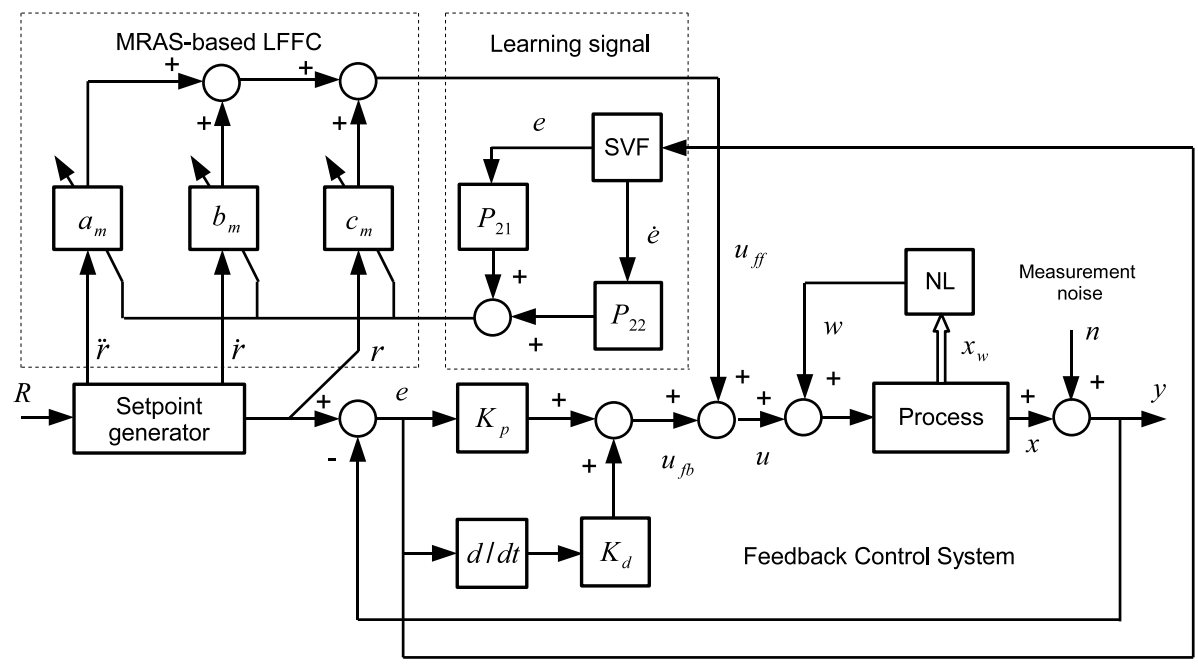

Figure 4.5: MRAS-based LFFC controlled system

$\omega_{n}=10[\mathrm{rad} / \mathrm{s}]$, and $\varsigma=1$, such that:

$$
\begin{aligned}
& A_{m}=\left[\begin{array}{cc}
0 & 1 \\
-\omega_{n}^{2} & -2 \varsigma \omega_{n}
\end{array}\right]=\left[\begin{array}{cc}
0 & 1 \\
-100 & -20
\end{array}\right], \\
& B_{m}=\left[\begin{array}{c}
0 \\
\omega_{n}^{2}
\end{array}\right]=\left[\begin{array}{c}
0 \\
100
\end{array}\right] .
\end{aligned}
$$

State variable filter: As mentioned in Section 4.3, the derivative of the error can be created using a state variable filter. The parameters of this state variable filter are chosen in such a way that the parameters of the reference model can vary without the need to change the parameters of the state variable filter every time $[42,22]$. The parameters are chosen as: $\omega_{n}=1000[\mathrm{rad} / \mathrm{s}]$ and $\varsigma=0.7$, then

$$
\begin{aligned}
& A_{F}=\left[\begin{array}{cc}
0 & 1 \\
-\omega_{n}^{2} & -2 \varsigma \omega_{n}
\end{array}\right]=\left[\begin{array}{cc}
0 & 1 \\
-10^{6} & -1.4 * 10^{3}
\end{array}\right], \\
& B_{F}=\left[\begin{array}{c}
0 \\
\omega_{n}^{2}
\end{array}\right]=\left[\begin{array}{c}
0 \\
10^{6}
\end{array}\right] .
\end{aligned}
$$

The learning signal for the adaptive components is given

$$
u_{\text {Learning }}(k)=p_{21} e(k)+p_{22} \dot{e}(k) .
$$

- $Q_{d}$ is chosen positive definite matrix and $P$ is solved from Equation (4.28). The following numerical values are used:

$$
Q_{d}=\left[\begin{array}{cc}
100 & 0 \\
0 & 100
\end{array}\right], T_{s}=0.001
$$




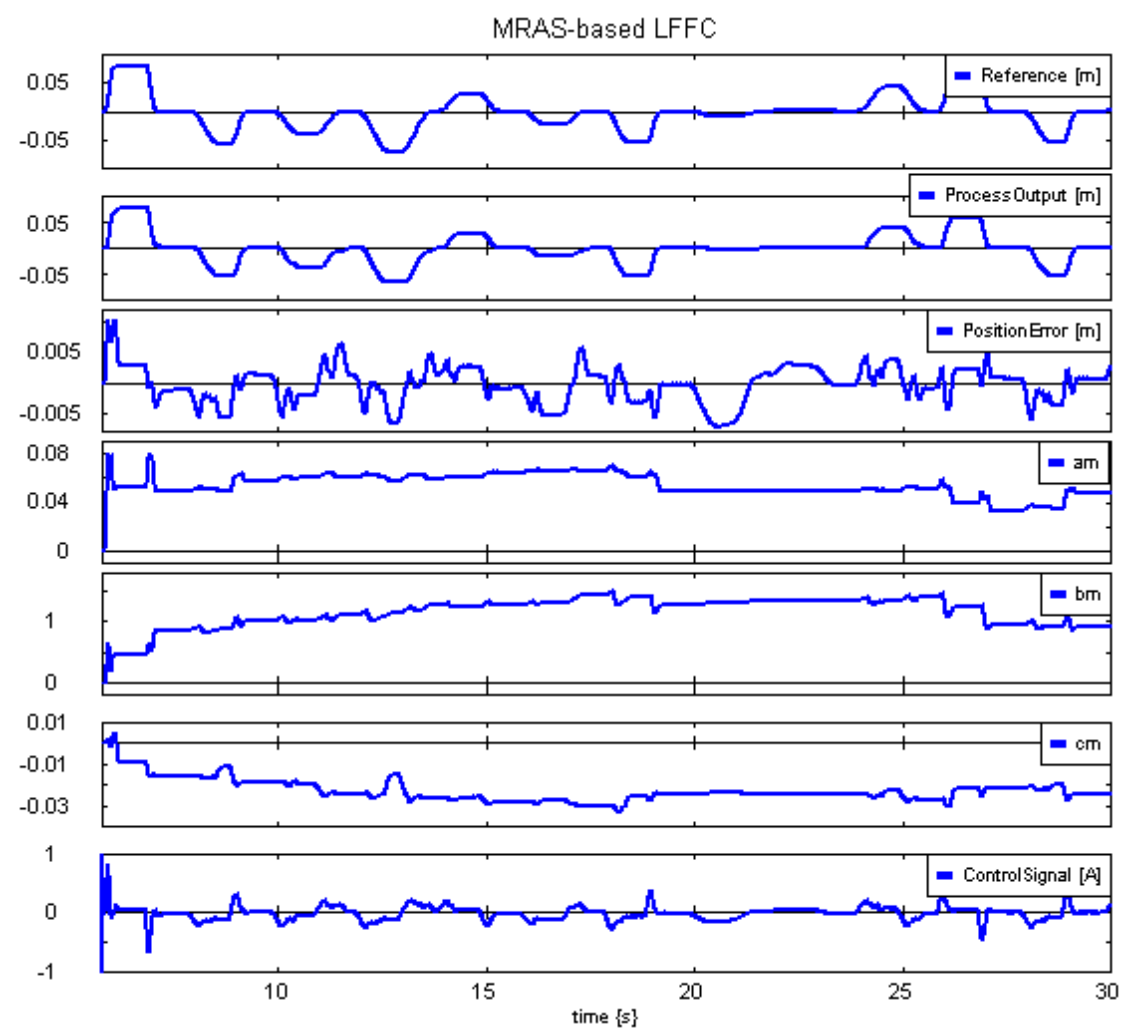

Figure 4.6: Simulation results when the feedback controller is switched off

These settings lead to the following Liapunov gains:

$$
p_{21}=0.3785, p_{22}=0.8359 \text {. }
$$

- The adaptive gains determine the speed of adaptation and they can be arbitrary chosen. The following settings are chosen $\left(1 / \alpha_{33}\right)=100,\left(1 / \alpha_{22}\right)=25000$, and $\left(1 / \alpha_{11}\right)=20$.

- The fixed feed-forward parameters are chosen initially, the learning process then makes them effective for compensating the disturbances. In this simulation, the initial values of the feed-forward gains are chosen $a_{m}(0)=b_{m}(0)=c_{m}(0)=0$.

Fig 4.6 shows the corresponding responses for the system of Fig 4.5. The adaptive feed-forward controller automatically finds the right parameters $\left(a_{m}, b_{m}\right.$ and $c_{m}$ ) to result in the stable system and properly generate the inversion of the process model. There are two major advantages of the MRAS-based LFFC compared with control based on feedback from the output. First of all, this structure can eliminate the influence of measurement noise by switching off the feedback 


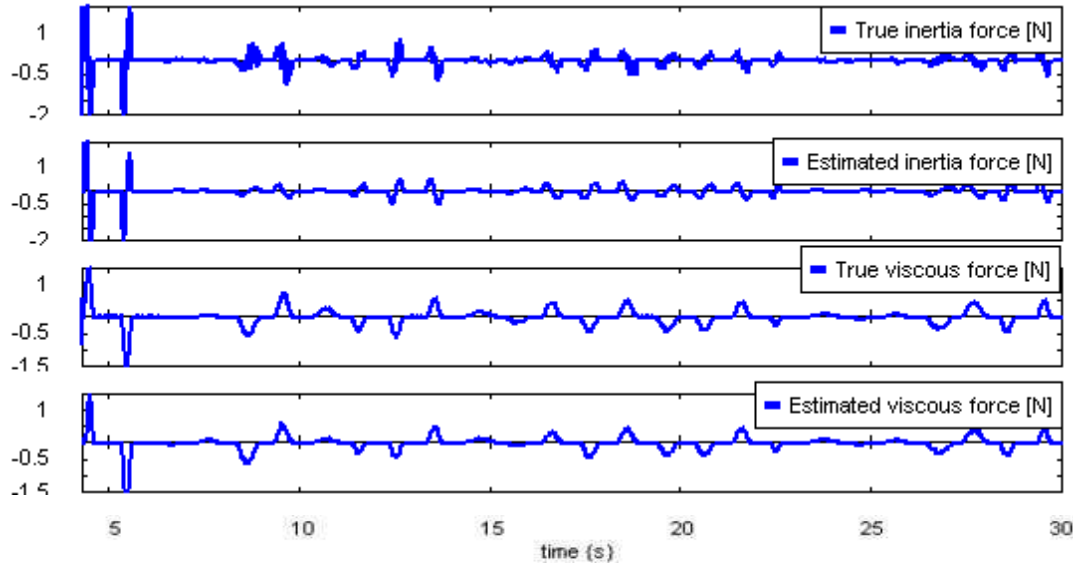

Figure 4.7: Linear disturbances are well compensated

controller completely, or almost completely [43]. In the ideal case, the effects of the measurement noise can then be totally eliminated. Furthermore, this controller

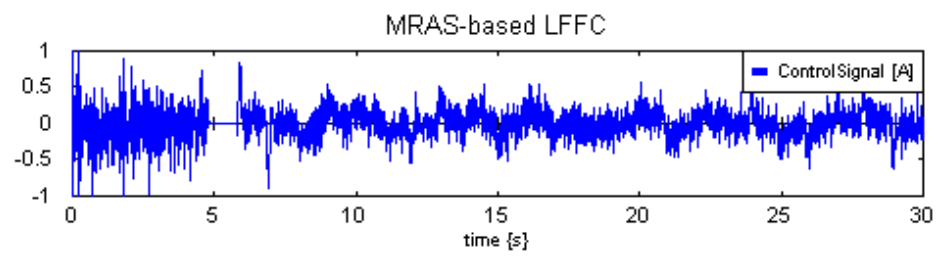

Figure 4.8: When the feedback controller is added, the control signal is corrupted by measurement noise

can reduce the influence of some disturbances. Such a structure can considerably improve the performance of the controlled system with respect to reference changes and plant parameter variations. An MRAS-based LFFC deals much better with noisy measurements than direct MRAS that has already been discussed in Chapter 2. The simulation results indicated in Fig 4.7 show that the inertia force and viscous friction force are well compensated by the feed-forward signals. This structure is suitable for the control of linear systems, or systems that can be approximated as such. It is very robust [43]. Also note that this $2^{\text {nd }}$ order LFFC is able to compensate the dynamics of $6^{\text {th }}$ order process.

However, it is clear that, when used alone a feed-forward controller cannot effectively compensate every disturbance, and robust stability cannot be guaranteed. When a feedback controller is used, sensor noises may be amplified and deteriorate performance. It is clearly observed in Fig 4.8 that the control signal is corrupted by measurement noise. 


\subsubsection{Discrete LQG design}

The design procedure for the discrete LQG controlled system based on the $2^{\text {nd }}$ order linear process model was introduced in Chapter 2. In this section we carry out the design with the $4^{\text {th }}$ - order linear process model. The LQG controller should be good enough to stabilize the process when used alone, but when combined with a feed-forward controller it does not need to minimize the position tracking error.

Discrete state matrices of the plant: The linear plant model to be controlled is

$$
\left\{\begin{array}{l}
x_{k+1}=A x_{k}+B u_{k} \\
y_{k}=C x_{k}+D u_{k},
\end{array}\right.
$$

in which (see Appendix A)

$$
\begin{gathered}
A=\left[\begin{array}{cccc}
1.0000 & 0.0000 & -0.0100 & 0.0000 \\
0.0010 & 1.0000 & 0.0000 & 0.0000 \\
0.0075 & 7.5000 & 0.9787 & -7.5000 \\
0.0000 & 0.0000 & 0.0010 & 1.0000
\end{array}\right], B=\left[\begin{array}{l}
0.0190 \\
0.0000 \\
0.0261 \\
0.0000
\end{array}\right] \\
C=\left[\begin{array}{llll}
0 & 1 & 0 & 0 \\
0 & 0 & 0 & 1
\end{array}\right], D=\left[\begin{array}{l}
0 \\
0
\end{array}\right] . \\
x=\left[\begin{array}{llll}
v_{L} & x_{L} & v_{F} & x_{F}
\end{array}\right]^{T} .
\end{gathered}
$$

In formula (4.40) $v_{L}$ is the velocity of the load, $x_{L}$ denotes the position of the load, $v_{F}$ is the velocity of the frame, and $x_{F}$ denotes the position of the frame.

Discrete optimal state feedback (LQR): The output of the state feedback controller is

$$
u=-K x
$$

in which $x$ represents the state of the system and $K$ is the gain vector based on the linear process model and optimization criterion

$$
K=\left(B^{T} P B+R_{R}\right)^{-1} B^{T} P A,
$$

where $P$ is the solution of the reduced matrix Riccati equation

$$
A^{T} P A-P-A^{T} P B\left(B^{T} P B+R_{R}\right)^{-1} B^{T} P A+Q_{R}+P=0 .
$$

In this equation $A$ and $B$ are discrete state matrices of the plant to be controlled, $Q_{R}$ and $R_{R}$ are matrices in the optimization criterion $\left(Q_{R}\right.$ is positive semi-definite weighting matrix and $R_{R}$ is positive definite weighting matrix). The following 


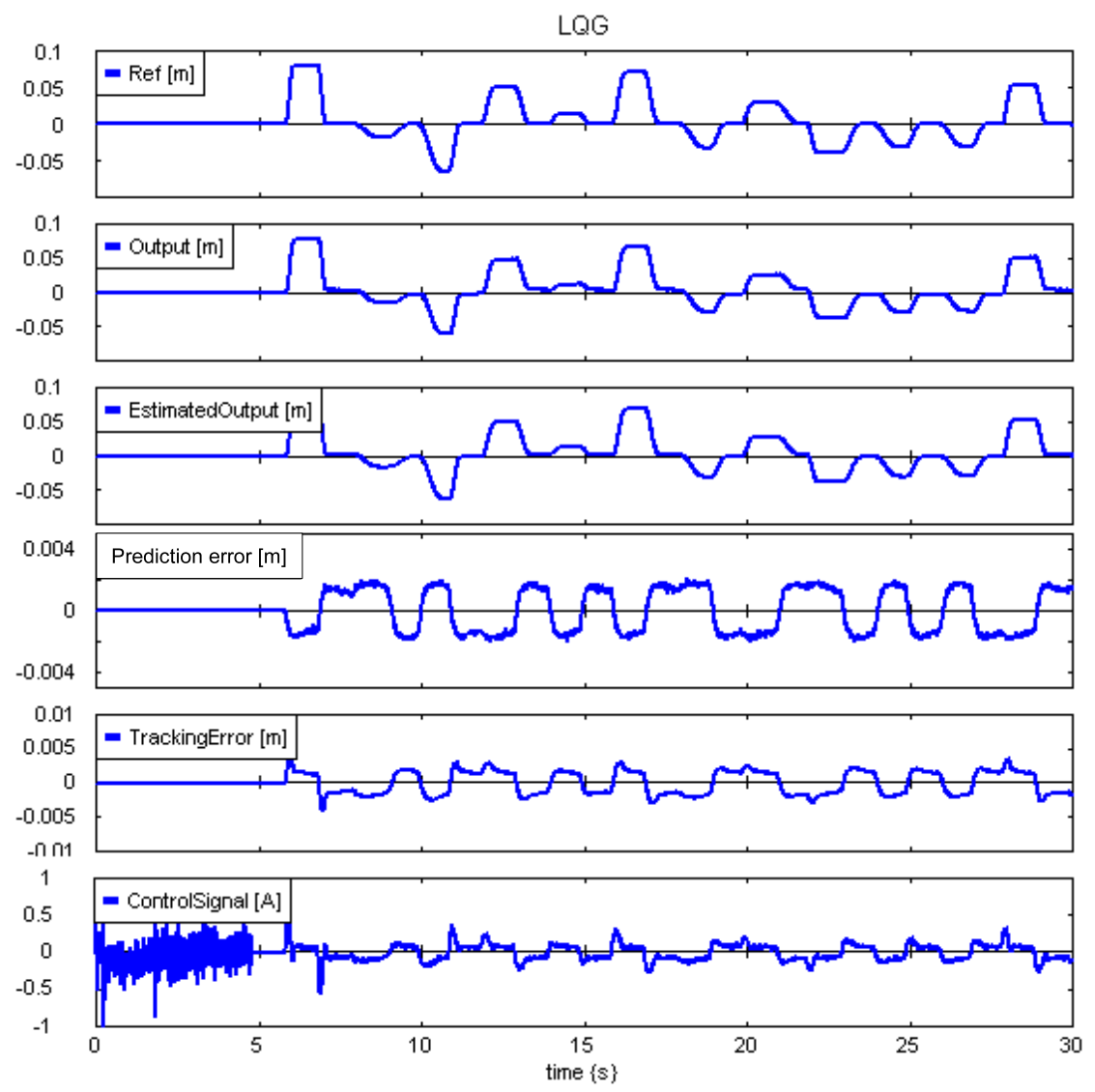

Figure 4.9: Estimated states and control signal are insensitive for measurement noise

settings are used

$$
Q_{R}=\left[\begin{array}{cccc}
0 & 0 & 0 & 0 \\
0 & 100 & 0 & 0 \\
0 & 0 & 0 & 0 \\
0 & 0 & 0 & 100
\end{array}\right], R_{R}=0.5
$$

These values result in the following stationary feedback controller gains:

$$
K_{L Q R}=\left[\begin{array}{llll}
1.008 & 9.412 & 0.010 & 10.38
\end{array}\right] .
$$

Discrete Linear Quadratic Estimator (LQE) : The feedback matrix $L$ yielding optimal estimation of the process states is defined as

$$
L=P C^{T}\left(C P C^{T}+R_{E} I\right)^{-1},
$$


where $P$ is the solution of the following matrix Riccati equation

$$
\operatorname{next}(P)=A(I-L C) P A^{T}+Q_{E},
$$

in which $A$ and $C$ are discrete state matrices of the plant to be controlled, $Q_{E}$ is the system noise covariance, and $R_{E}$ the sensor noise covariance. The following settings were used

$$
Q_{E}=\left[\begin{array}{cccc}
10 & 0 & 0 & 0 \\
0 & 100 & 0 & 0 \\
0 & 0 & 100 & 0 \\
0 & 0 & 0 & 10
\end{array}\right], R_{E}=1 .
$$

The settings result in the following stationary gains

$$
L_{L Q E}=\left[\begin{array}{cc}
0.3048 & -0.0059 \\
0.9167 & 0.0000 \\
0.6294 & -0.3915 \\
0.0000 & 0.9160
\end{array}\right] .
$$

The simulation results of the LQG controlled system are shown in Fig 4.9. As can be seen, noise on the measurements of the process has almost no influence on the system. The real position measurement (second line) and the position state error (fourth line) are corrupted by measurement noise, whereas, the estimated position state (third line) and the control signal (lowest line) are almost clean.

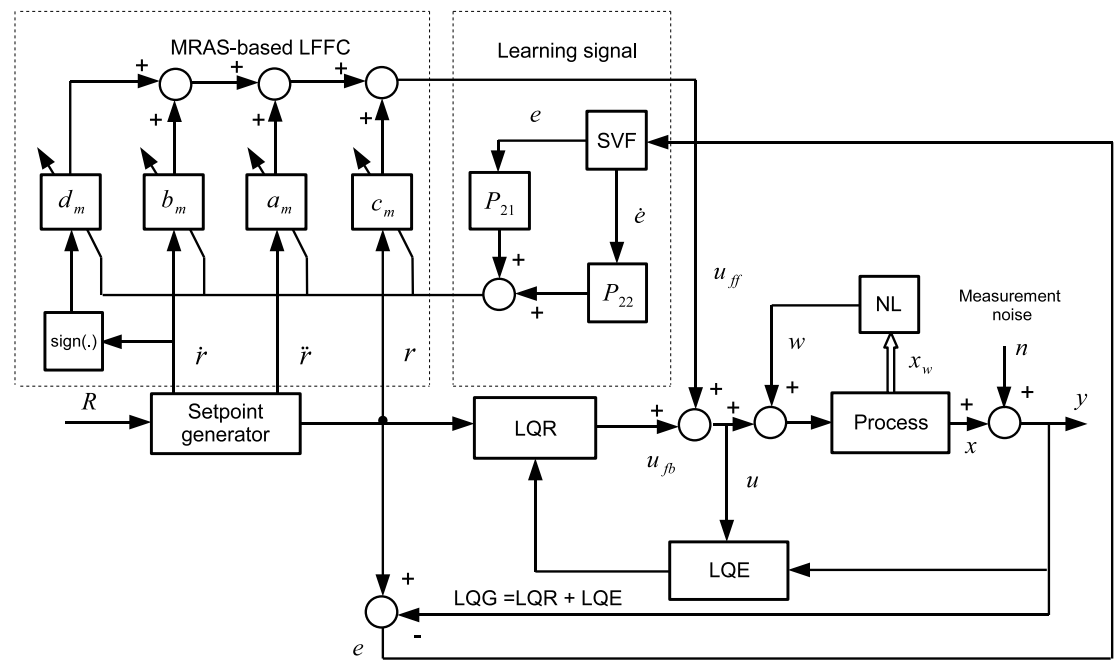

Figure 4.10: The proposed control structure with Coulomb friction compensation 


\subsubsection{LQG combined with MRAS-based LFFC with friction compensation}

With the MRAS-based learning feed-forward control as given in Section 4.2, only compensation for the effect of the viscous friction is mentioned. In order to improve tracking systems capabilities we have to take the effect of the Coulomb friction into account. This is can be realized by adding an extra learning component in the learning feed-forward part (See Fig 4.10). The Coulomb friction force depends on the direction of the velocity, therefore, the adjustable gain has to be dependent on the sign of the velocity. Thus the adjustment rule for the adjustable parameter to compensate the Coulomb friction is

$$
d_{m}=\frac{1}{\theta} \Sigma\left[\left(p_{21} e+p_{22} \dot{e}\right) \operatorname{sign}\left(x_{m, 2}\right)\right] T_{s}+d_{m}(0)
$$

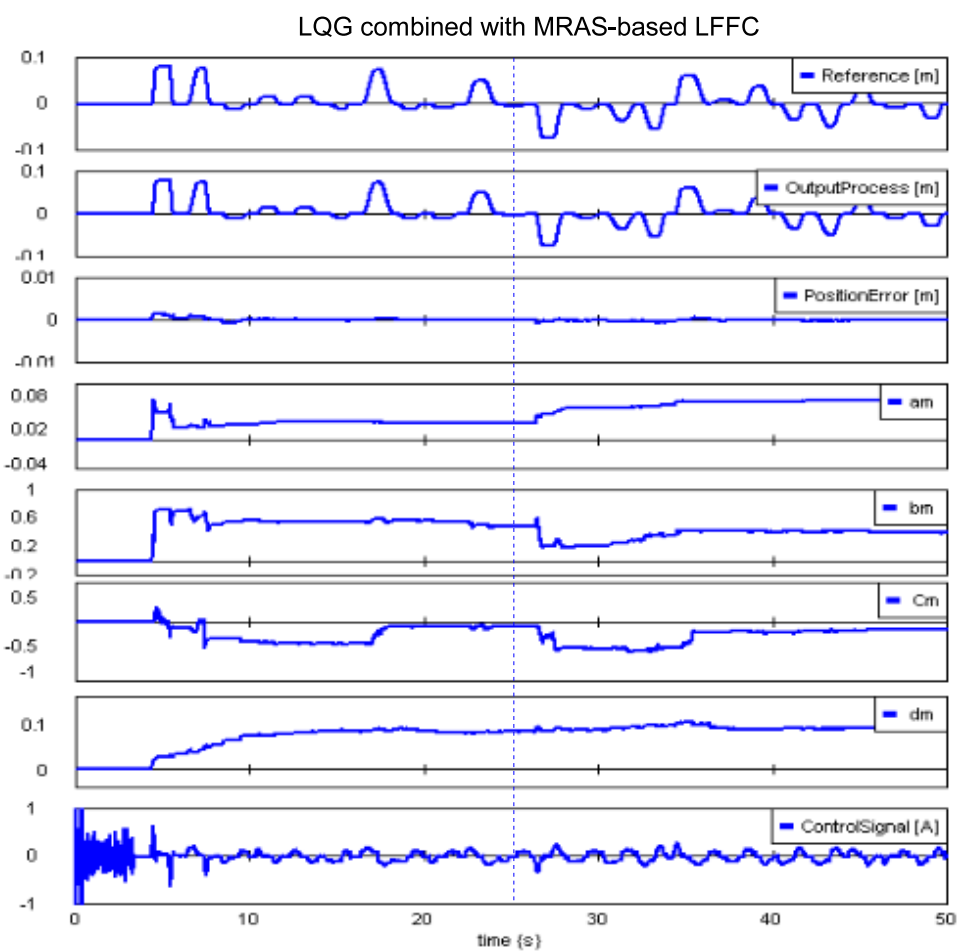

Figure 4.11: Simulation results in the presence of measurement noise and mass variation

The adjustment of the parameter depends on the sign of $x_{m, 2}$, which is the reference velocity. To illustrate the design method, the values of the simulation 
parameters are used as given in the previous simulations and the value of the adaptive gain of the Coulomb friction compensation is chosen as $(1 / \theta)=3000$.
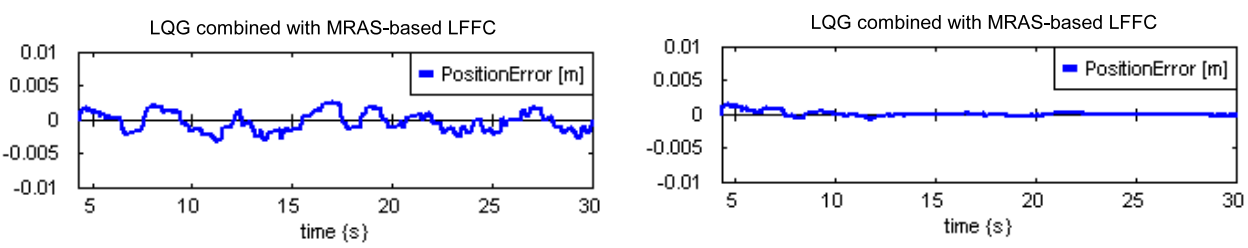

Figure 4.12: Position error without (left) and with (right) Coulomb friction compensation

To illustrate the performance of the controller in the presence of measurement noise and plant parameter variations, the following numerical values are used: an input reference (with stroke $R=0.1[\mathrm{~m}]$, measurement noise on slider position (with amplitude $A=0.0001[\mathrm{~m}]$ ), and a parameter variation in the load (with mass variation $\Delta_{m}=0.1[\mathrm{~kg}]$. The experiments consist of three phases: $t=0-5[s]$; homing operator, the system is controlled by a fixed PID controller; $t=5-25[\mathrm{~s}]$; normal parameter, the system is controlled by the proposed controller; $t=25-50[\mathrm{~s}]$; at $t=25[\mathrm{~s}]$ a parameter variation is added to the load of the plant. The simulation results (see Fig 4.11) show that when a mass varia-

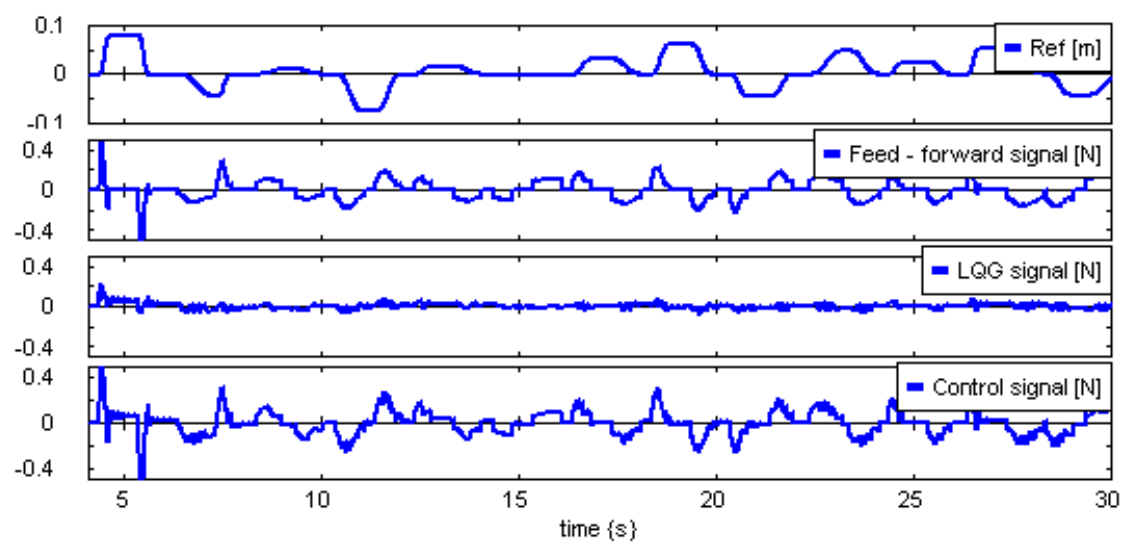

Figure 4.13: Feed-forward and LQG output signals

tion of the load is switched on, after a few motions, the feed forward controller is able to give a good control signal for the process. The parameters $a_{m}, b_{m}, c_{m}$ and $d_{m}$ quickly search the new stationary values. The influence of measurement noise is well eliminated. It is observed that the control signal is almost clean. By combining LQG and MRAS-based LFFC, it is possible to improve both tracking errors and the measurement noise rejection property. The compensation of the 

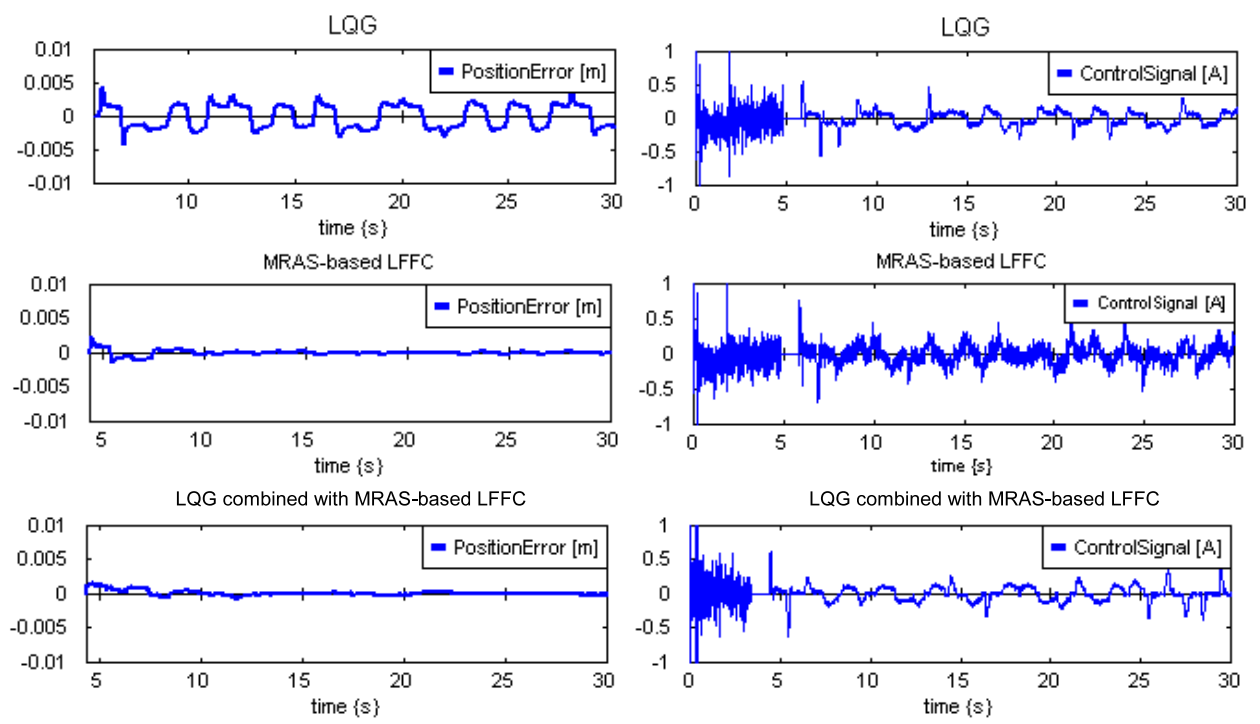

Figure 4.14: Comparison of the controllers

Coulomb friction force can be clearly observed in Fig 4.12. Without Coulomb friction compensation, the effect of friction can be seen by a large tracking error. The maximum tracking error in the case of stationary gains will be $0.3[\mathrm{~mm}]$. When the adaptive Coulomb friction compensator is used, the effect of friction was compensated considerably. It can be seen that the convergence is faster and the tracking error will converge to a smaller value. The maximum tracking error in the case of stationary gains will be $0.02[\mathrm{~mm}]$. It can be stated that in motion control systems, Coulomb friction compensation is the key factor to obtain small tracking errors. The control signal is the sum of the feedback signal and the feed-
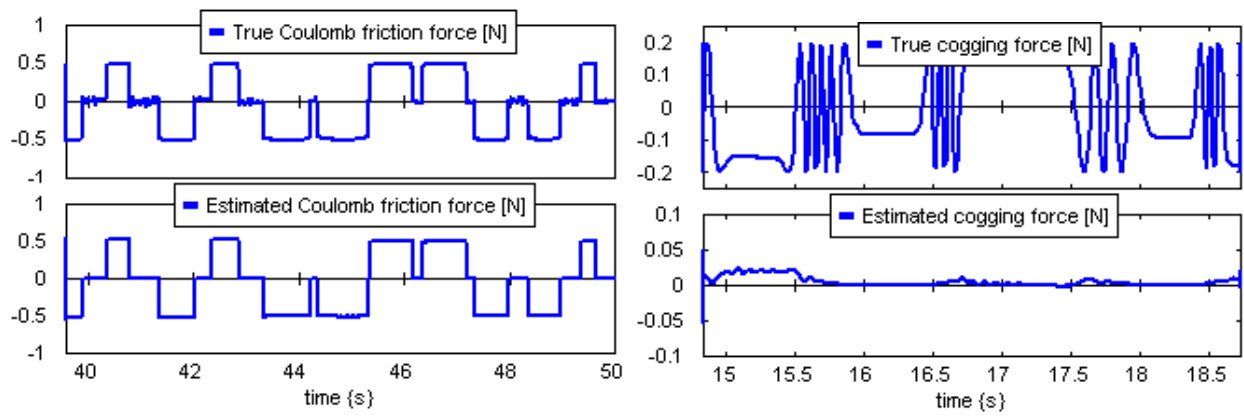

Figure 4.15: (Left) Coulomb friction force is well compensated by adaptive $d_{m}$; (Right) Adaptive $c_{m}$ fails to compensate the cogging force 
forward signal. As can be seen in Fig 4.13, in the first run, the control signal is determined by both the control signals, whereas, in the second run, it is determined almost completely by the feed forward controller. The combined controller is superior to the two methods when used separately (see Fig 2). The advantages of both methods are combined, whereas, the disadvantages are eliminated.

\subsubsection{LQG combined with MRAS-based LFFC with cogging compensation}

As introduced in Chapter 3, for the MeDe5, the cogging phenomenon is insignificant such that can be ignored. However, in order to illustrate the effectiveness of the proposed control structure, cogging force is added in the plant model. In this simulation, we assume that the cogging force has a magnitude of $0.2[N]$, and a period of $0.005[\mathrm{~m}]$. It was modeled as:

$$
F_{C}(x)=0.2 \sin \left(\frac{2 \pi}{0.005} x\right)=0.2 \sin (1256 x)[N]
$$

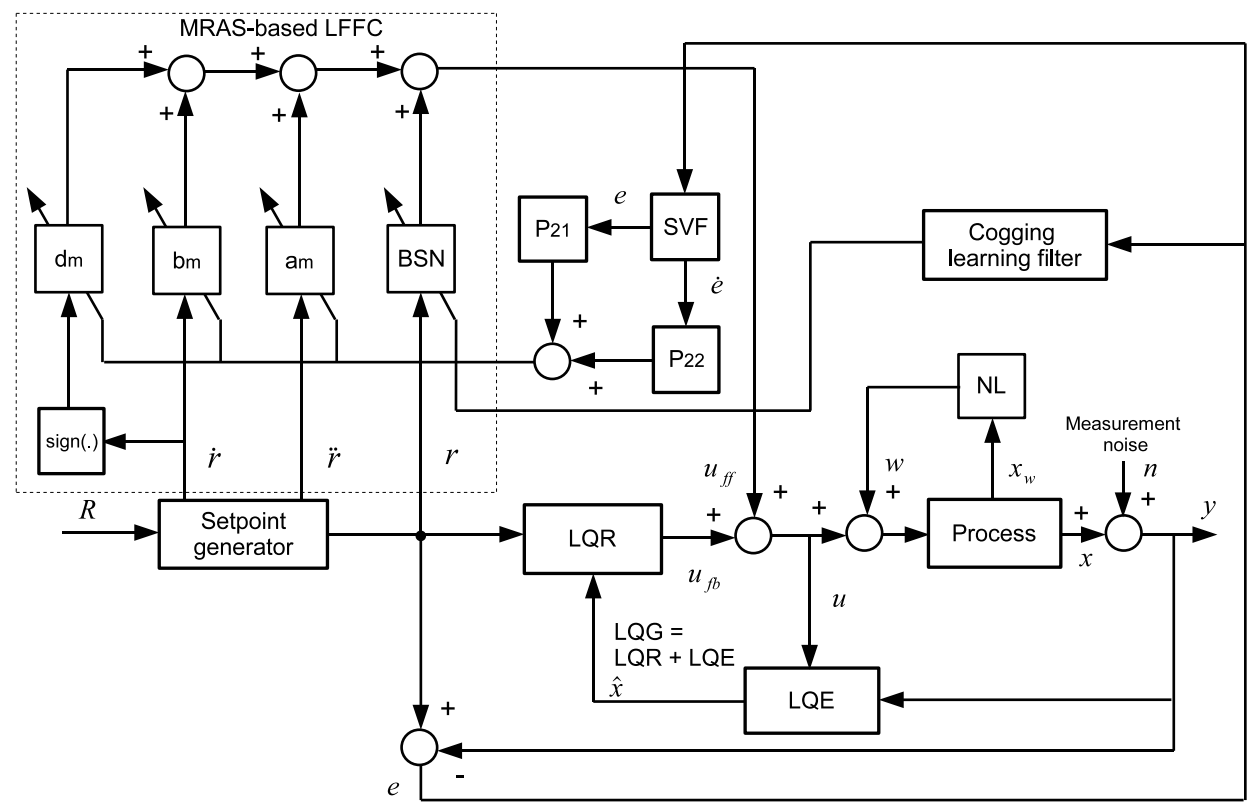

Figure 4.16: B-spline neural network is used to compensate the cogging force

The adaptive $c_{m}$ that has the reference position as input has to compensate the cogging force. However, with the proposed control structure, the simulation 
results show that the adaptation if $c_{m}$ is not fast enough such that the cogging force could not be compensated (see Fig 4.15). To overcome this problem, the solution is that the adaptive $c_{m}$ should be replaced by a B-spline neural network (BSN) with a separate learning signal, as shown in Fig 4.16. The parameters of this BSN are indicated in Chapter 3. In Fig 4.19, with an adaptive cogging compensator, the effect of cogging force could not be compensated. It results in a large tracking error. When the adaptive cogging compensator is replaced by the BSN, the effect of cogging force was compensated significantly (see Fig 4.18 and Fig 4.19). After a few motions, the feed forward controller is able to give an optimal control signal for which the process output will track the desired reference with a small error.

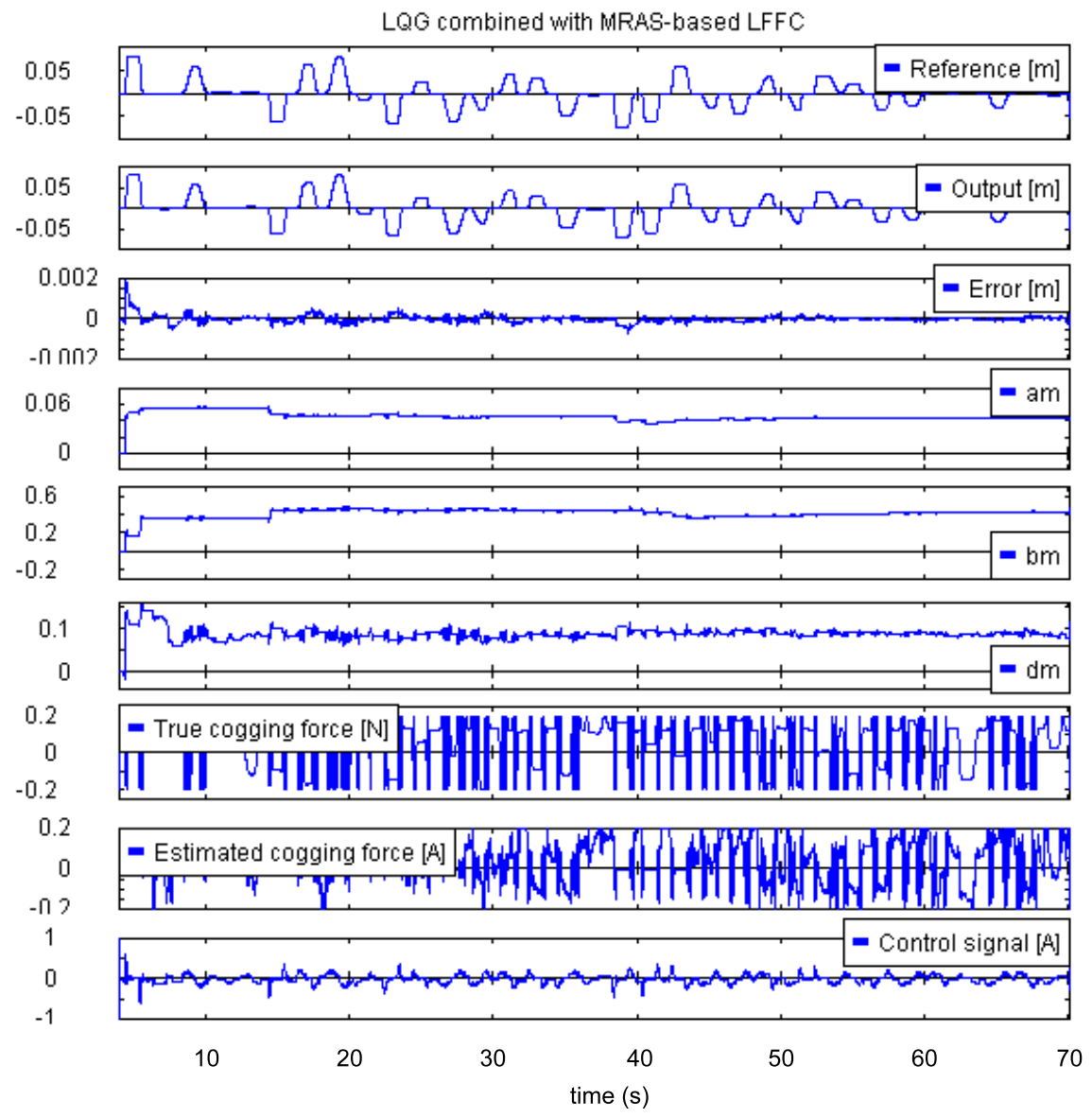

Figure 4.17: Simulation results when the BSN is used 


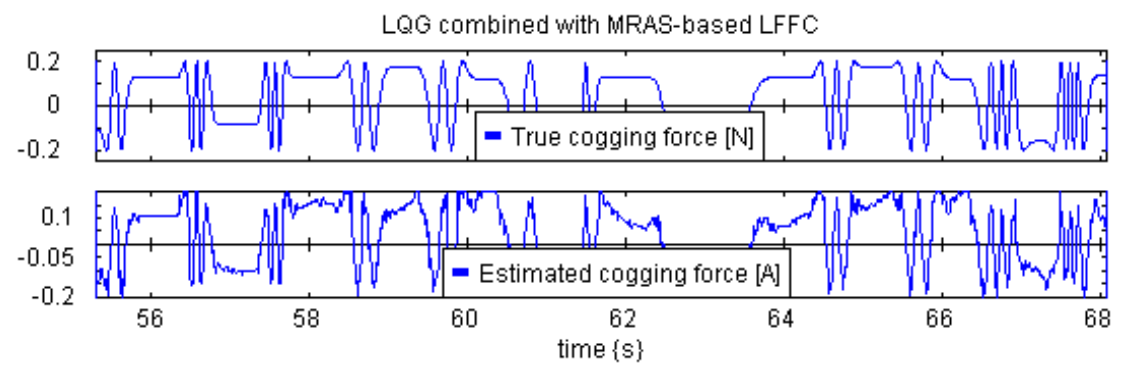

Figure 4.18: The BSN well compensates for the cogging force

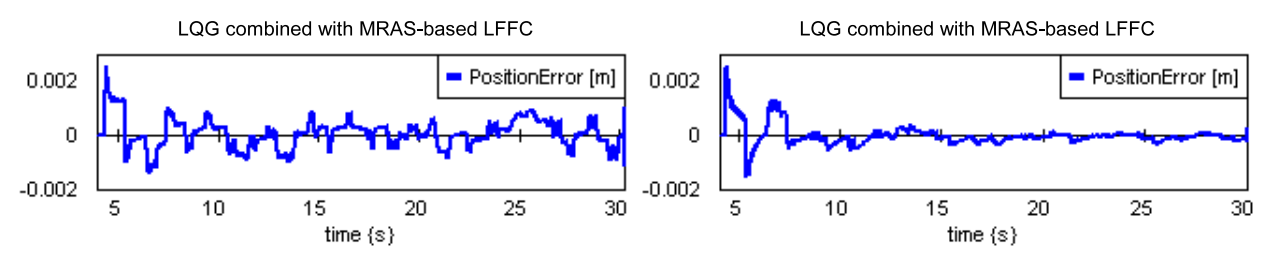

Figure 4.19: Position error without (left) and with (right) cogging compensation

\subsection{The LQG combined with MRAS-based LFFC controller compared with the PID controller}

The performances of the proposed controller and a conventional PID controller are compared in the presence of measurement noise and plant parameter variations. A measurement noise on the slider position with amplitude $A=0.0001[\mathrm{~m}]$ is added at $t=0[\mathrm{~s}]$, and a mass variation is added to the load of the plant at $t=25[\mathrm{~s}]$. The parameters of both controllers are given in previous sections. By comparing two simulation results as indicated in Fig 4.20, the main advantages of the proposed controller are clearly shown: (1) The proposed structure exhibits smaller tracking errors; (2) The system dynamics are relatively insensitive to changes in system parameters; (3) The control signal is relatively insensitive to measurement noise. With an MRAS-based LFFC combined with LQG, it is thus possible to obtain accurate control of a given plant, whereas with doing so is impossible in conventional control structures.

\subsection{Discussion}

1. In Section 4.2, the design of an MRAS-based LFFC was carried out with the second-order example, however the approach can be effectively applied to higher order systems as well. This was demonstrated by using a second- 

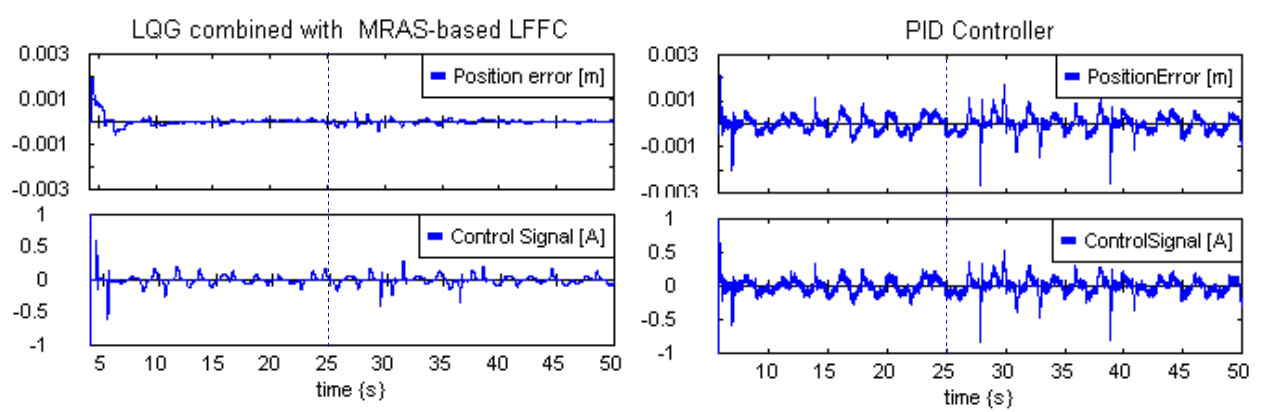

Figure 4.20: Comparison of the controllers

order controller structure for the control of a sixth-order process.

2. The advantages of the use of the profile setpoint signals is that they are easily accessible and noise free.

3. The design is based on the assumption that the difference between the reference state and the process state is small. If it would be large the inverse model of the plant cannot be realized in the feed-forward controller.

4. The adjustable parameter component has an integral component inside. This implies that even when the learning signal is corrupted by measurement noise the output signal is almost clean. This allows us using a large learning signal to shorten the setting time.

5. In case of an MRAS-based LFFC, when all disturbances can be effectively compensated for by a feed-forward signal, this allows us to reduce the values of the feedback controller gains. In this case measurement noise has almost no influence on the system.

6. The parameters of the LQG controller are given fixed values, corresponding with the nominal values of the plant. With the parameter variations of the plant considered here it appeared that the LQG was robust enough to deal with these variations and to produce good enough results for the basic feedback control system.

\subsection{Conclusions}

The plant is always subject to disturbances, model uncertainty, and measurement noise. The nature of the disturbances affects the quality of performance in a controlled system. Un-modeled dynamics and parametric uncertainty have a negative 
effect on the tracking performance and can even lead to instability. Measurement noise limits the achievable bandwidth of the closed-loop system. In order to reduce the effects of unwanted factors as mentioned we have to take them into account in the design procedure. The solution is in the form of a two-degrees-offreedom controller, which consists of an LQG controller and a separate MRASbased LFFC.

When LQG is used alone a compromise has to be made between performance and robustness specifications. The feed-forward may help to overcome this difficulty. As expected, a combined LQG and MRAS-based LFFC exhibits small tracking error, as well as good measurement noise rejection properties in the presence of plant parameter variations and measurement noise. The effectiveness of the controller is also illustrated through the comparison, obtained by observing the simulation results, when using PID controller. The control ability of the proposed structure is greatly improved when the adaptive feed-forward $c_{m}$ is replaced by the corresponding B-spline neural network element. The unknown cogging force is well compensated in the feed-forward controller. From results obtained of this study we can state that the combination between LQG and MRAS-based LFFC is an attractive approach for controlling electromechanical motion systems that are subject to reproducible disturbances, measurement noise, and model uncertainty. 


\section{Chapter 5}

\section{Applications}

\subsection{Introduction}

In the previous chapters, several control algorithms for electromechanical motion systems have been introduced. We have performed experiments to confirm the effectiveness of the proposed control methods to put them to practical use. The obtained knowledge and insight will be applied to the Tripod and the MeDe5 setup. However, all of the control algorithms introduced in Chapter 2 (PID, LQG, Direct MRAS, Indirect MRAS, and STR) have already been tested on the MeDe5 $[14,44]$. Thus, in this chapter we concentrate only on experimental tests for the control algorithms introduced in Chapters 3 and 4.

In Section 5.2 the LQG combined with MRAS-based LFFC is tested on the MeDe5. We address the problem relating to the precision control of the DC motor, which requires the needs of mechanical transmission from the rotary to linear motion. Our main focuses are: (i) whether and how the compensation of state dependent effects, e.g. friction, mass deviation, can be realized by the MRASbased LFFC; (ii) how the attenuation of measurement noise, and the reduction of plant parameter variations, can be realized by LQG controller. Experimental tests will be carried out to demonstrate the performance difference between the system based on the LQG controller and that based on the addition of the MRAS-based LFFC. In Section 5.3 the parsimonious LFFC introduced in Chapter 3 and the MRAS-based LFFC introduced in Chapter 4 are tested on the Tripod. We address the problem relating to the precision control of the linear motor to track desired motion trajectories. Our main interest is whether and how the compensation of state dependent effects, e.g. friction, cogging and mass deviation, can be realized by LFFC. Thus, experimental tests will be carried out to demonstrate the performance difference between the system based on the feedback controller only and that based on the addition of the feed-forward controller. 


\subsection{Implementation for the MeDe5}

This section describes the testing of LQG combined with the MRAS-based LFFC control algorithm on the MeDe5. The total system for testing in practice is depicted in Fig 5.1. To demonstrate the usefulness and accuracy of the method,

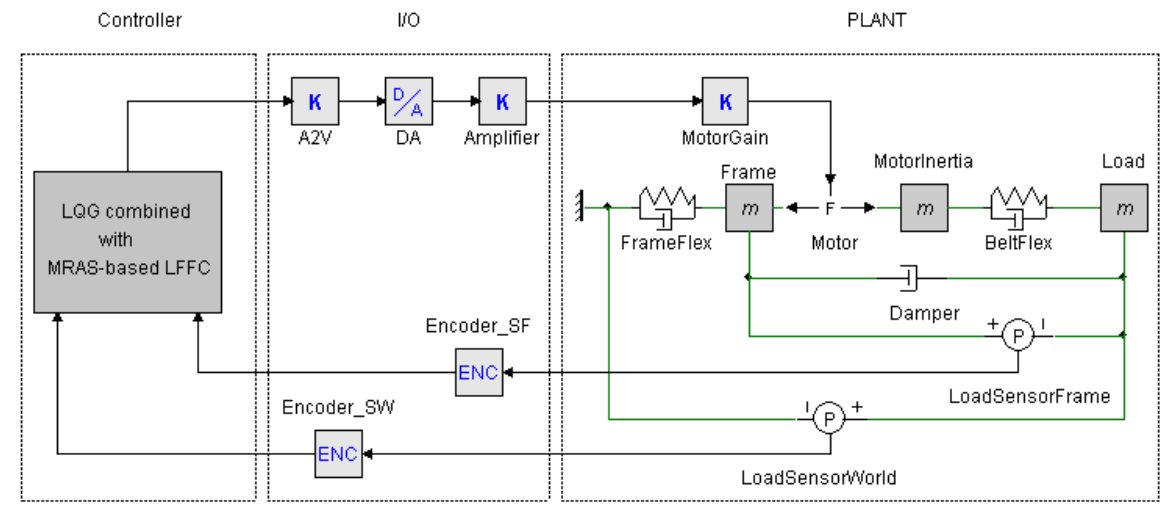

Figure 5.1: The MeDe5 with LQG combined with MRAS-based LFFC.

several experiments with the LQG controller alone and the addition of the MRASbased LFFC are performed. These experiments will completely follow the control designs introduced in Chapter 4. After generating and uploading the C-code of this controller into the PC104-board of the MeDe5, the performance of the system can be measured [14].

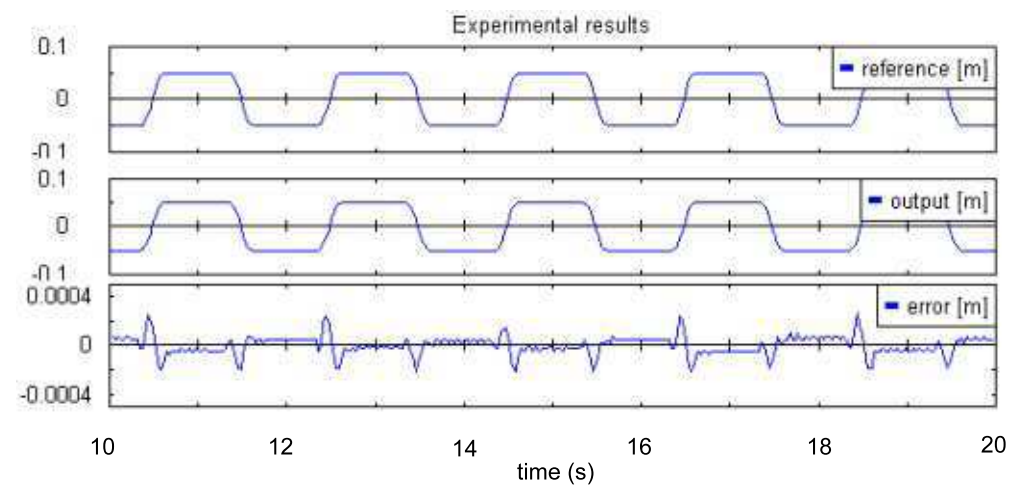

Figure 5.2: Experimental results when a repeating motion profile is used.

In the first experiment only the LQG controller is used. As can be seen in Fig 5.2, the LQG controlled system does not reach its reference values of $(-0.05 \mathrm{~m}$ and $0.05 \mathrm{~m})$. A considerable static error remains in the system due to friction. 
The value of the maximum tracking error is $0.25[\mathrm{~mm}]$. We expect, the remaining static error in the LQG system to be decreased by adding an MRAS-based LFFC to the LQG control algorithm.

Comparing the results of the LQG controlled system in simulation (see Fig 5.3 top) and in experiment (see Fig 5.3 bottom) it is observed that the maximum tracking error is significantly bigger in the simulation case. It can be explained that the mathematical model does not correspond the real process.
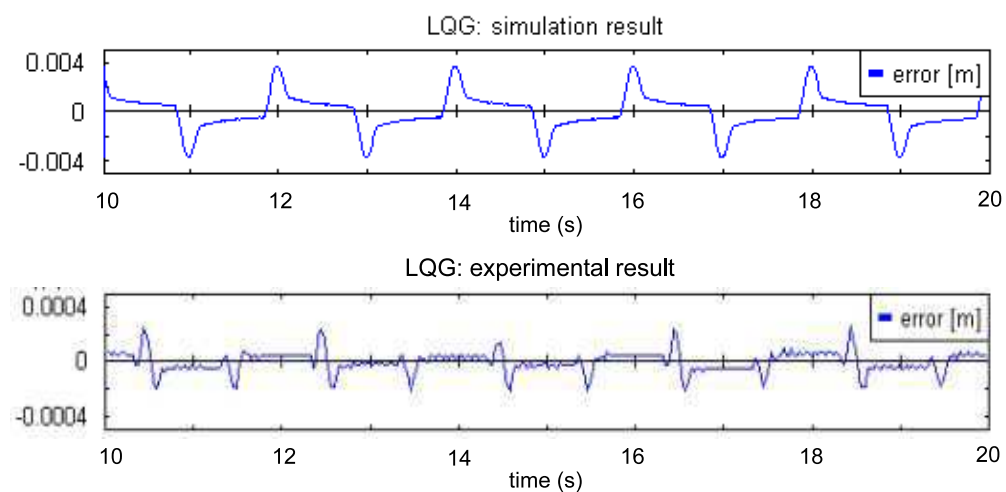

Figure 5.3: A comparison between simulation result and experimental result.

Next, the experimental test is repeated with a random motion profile, the maximum tracking error in this case will be as large as 0.5 [mm] (see Fig 5.4). Both in simulation and experimental results it is shown that the LQG controlled system operates better on a repeating motion than with a random motion.

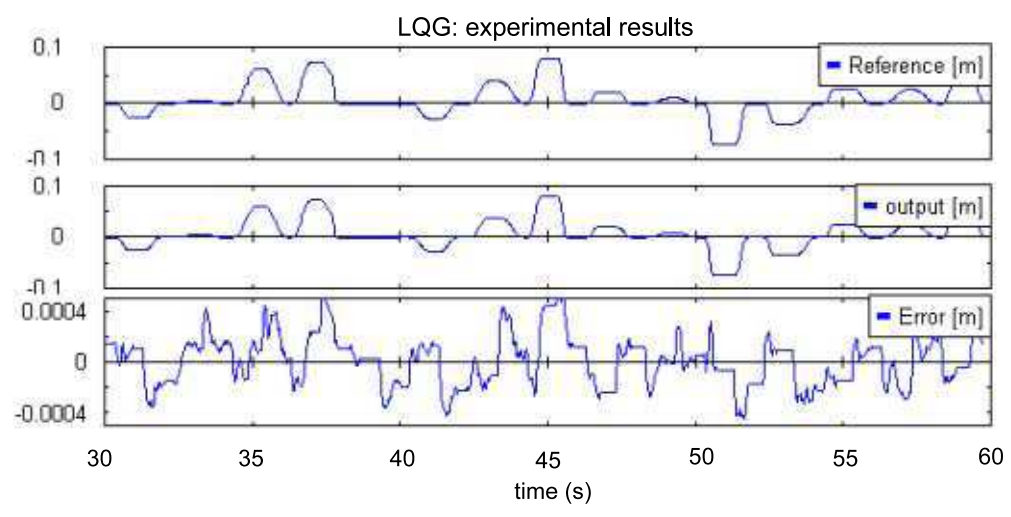

Figure 5.4: Experimental results when a random motion profile is used.

In the second experiment the MRAS-based LFFC is added to the LQG controller. The role of the MRAS-based LFFC is clearly shown by comparing two 
simulation results as indicated in Fig (5.5 top) and Fig (5.5 bottom). This addition significantly increases the performance of the controller: the large static error with the LQG controller almost disappears. The maximal tracking error with a repeating motion profile is about 0.035 [mm].
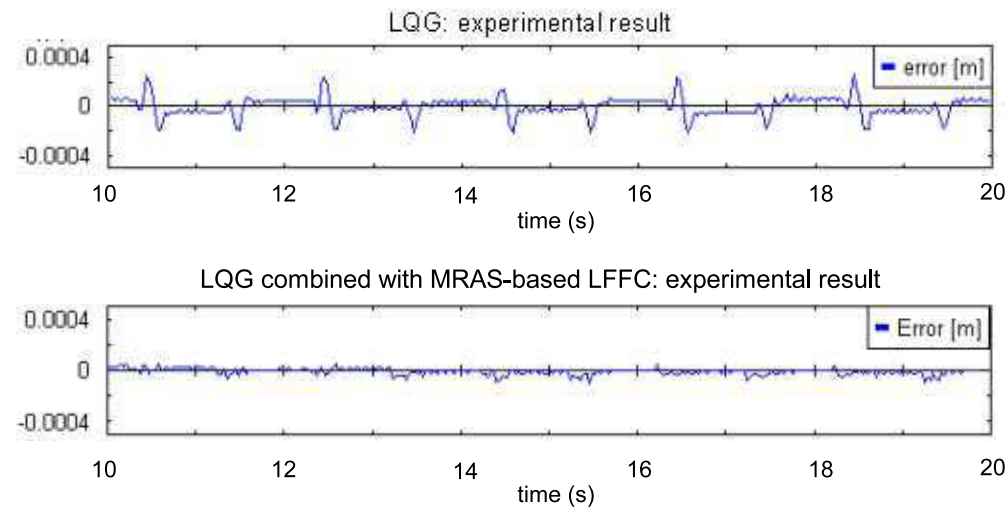

Figure 5.5: A comparison when a repeating motion profile is used.

The feed-forward parameters are shown in Fig 5.6 and the estimated disturbances in Fig 5.7. It is observed that the expected shapes of the estimated disturbances are obtained. In other worlds, the reproducible disturbances are well compensated by the feed-forward part.

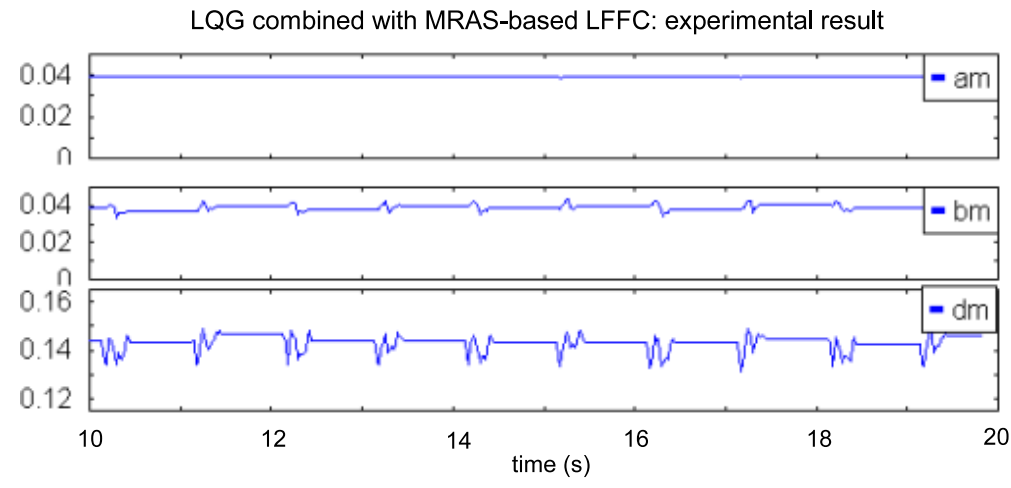

Figure 5.6: Feed-forward parameters.

The stability of the combination of an LQG controller and the MRAS-based LFFC was tested with a long time experiment. The system is stable and shows no drift in the parameters. It is confirmed by comparing two experimental results as indicated in Figures 5.6 and 5.8. The feed-forward parameters after 10 minutes 
and the corresponding feed-forward parameters after 60 minutes are almost the same.

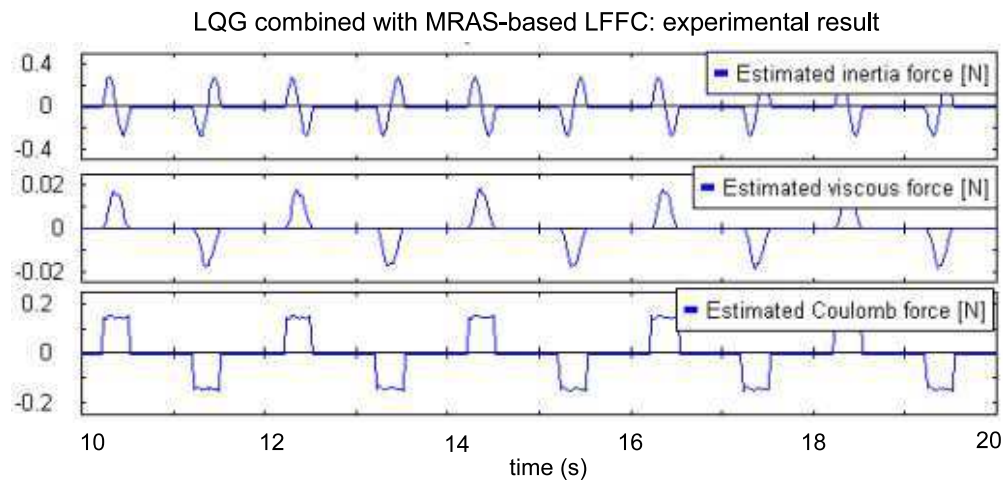

Figure 5.7: Estimated disturbances when a repeating motion profile is used.

Next, a random motion profile is used. The contribution of the MRAS-based LFFC is clearly shown. In Fig (5.9 top), without MRAS-based LFFC, the effect of reproducible disturbances will result in a large tracking error. However, when the MRAS-based LFFC is added, this effect is greatly compensated (see Fig 5.9 bottom). Since this was the primary goal of the feed-forward controller, it can be concluded that the benefit of the implementation of the MRAS-based LFFC has been successful. The value of the maximum tracking error is about $0.1[\mathrm{~mm}]$.

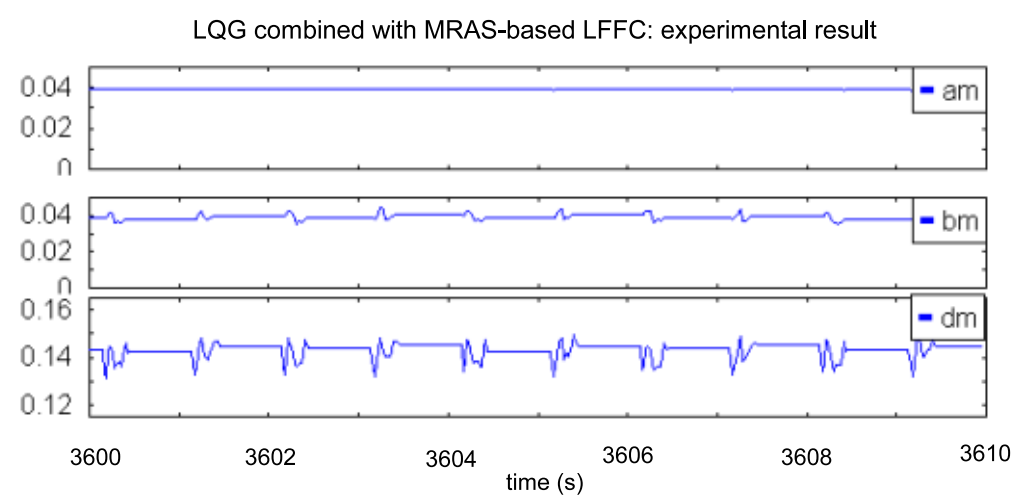

Figure 5.8: Long time experiment.

\section{Discussion}

1. When the LQG controller is used alone the tracking error is large. 


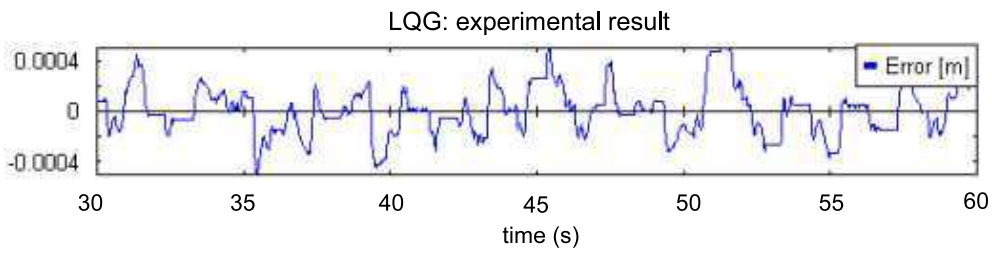

LQG combined with MRAS-based LFFC: experimental result

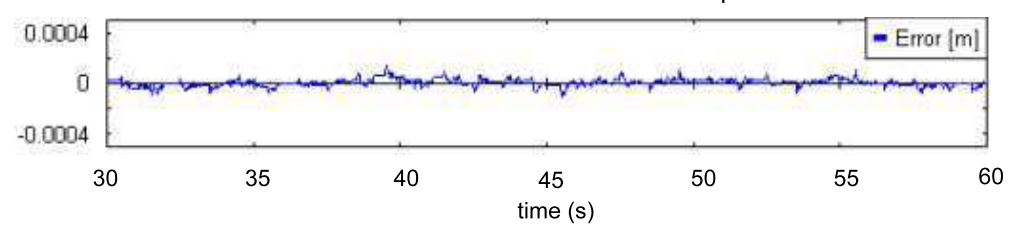

Figure 5.9: A comparison when a random motion profile is used.

2. The friction compensation in the MRAS-based LFFC makes a large enhancement on the behavior of the system. A combination of both the controllers will reduce the tracking error.

3. In order to obtain a faster rate of convergence the initial values of the adjustable feed-forward parameters should be chosen as close as possible to their corresponding final values.

4. The LQG combined with MRAS-based LFFC operates better on a repetitive motion instead of a random motion. It is proved in both simulation and experimental results.

5. Both simulation and experimental results are presented to verify the effectiveness of the proposed controller. The LQG combined with the MRASbased LFFC is able to obtain a 5 times smaller tracking error than the LQG controller alone. Moreover, the proposed controller also can obtain fast dynamic response and high stability. This is important for real-time control applications.

\subsection{Implementation for the Tripod}

In this section, we will apply NN-based LFFC and MRAS-based LFFC to the Tripod setup. The design of the parsimonious LFFC introduced in Chapter 3 and the design of the MRAS-based LFFC in Chapter 4 are repeated in short in Paragraphs 5.3.1 and 5.3.2, respectively. No detailed theoretical analyses are discussed in these paragraphs, however, main calculated results are given. The Tripod is a system controlled by a computer, which requires a discrete control algorithm [9] 
(see Fig 5.10 and Fig 5.13). In Section 5.3.3 the designed controllers will be tested on the real setup. The results are shown for different experiments to demonstrate the effectiveness of the proposed control schemes.

\subsubsection{Design of a parsimonious LFFC}

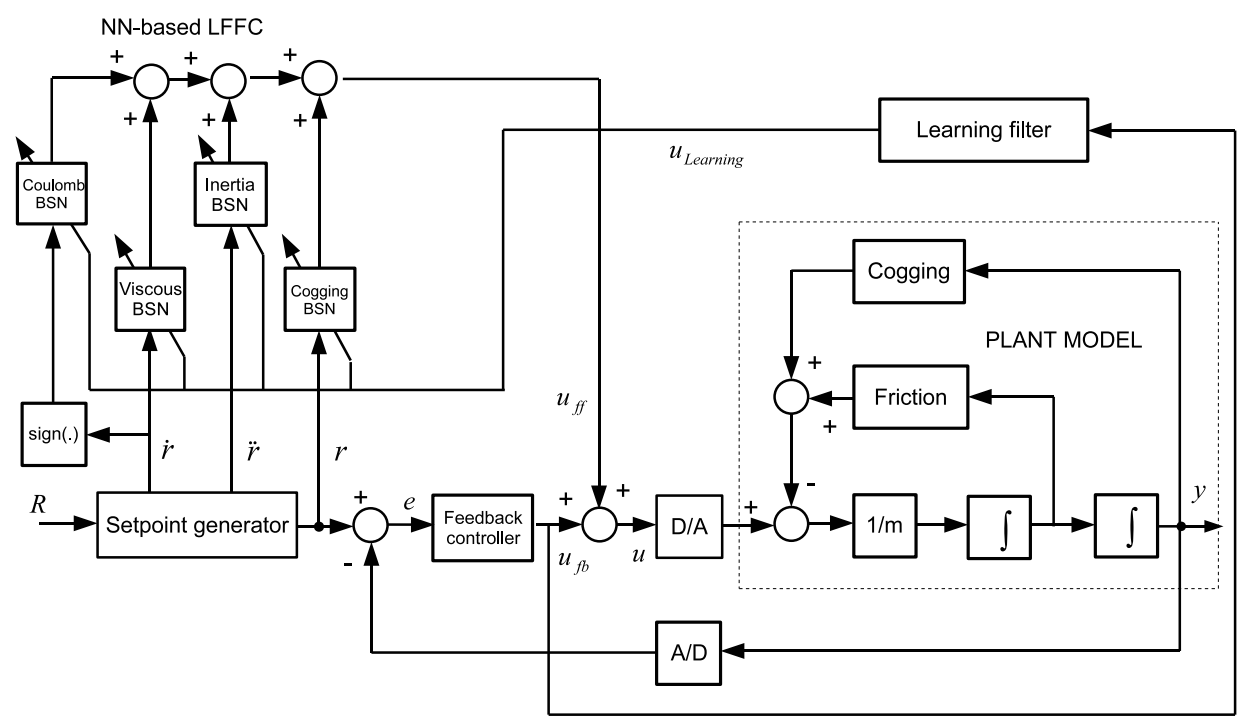

Figure 5.10: NN-based LFFC controlled system for each motor of the Tripod.

\section{Step 1: Design the feedback controller}

The behavior of each motor is modeled as a moving mass:

$$
P(s)=\frac{1}{m s^{2}},
$$

where $m(=6[\mathrm{~kg}])$ represents mass of the load. A PD-controller is chosen for the control of each motor. The transfer function of the controller is given by [9]:

$$
C(s)=K_{p} \frac{s \tau_{d}+1}{s \beta \tau_{d}+1}
$$

where $K_{p}=809.10^{4}, \tau_{d}=8.43 .10^{-3}$, and $\beta=0.1$.

\section{Step 2: Determine the inputs of the feed-forward part}


In the Tripod the plant is subject to the following disturbances: unknown friction force, and unknown cogging force. That is, we should select $[r, \dot{r}, \operatorname{sign}(\dot{r}), \ddot{r}]$ as inputs of the feed-forward controller.

\section{Step 3: Choose the structure of the feed-forward part}

For the Tripod design, the solution to prevent the curse of dimensionality is given in Fig 5.10. The proposed network structure should consist of four BSNs that have the reference position $r$, velocity $\dot{r}$, sign of velocity, and acceleration $\ddot{r}$ as inputs.

\section{Step 4: Choose the B-spline distribution}
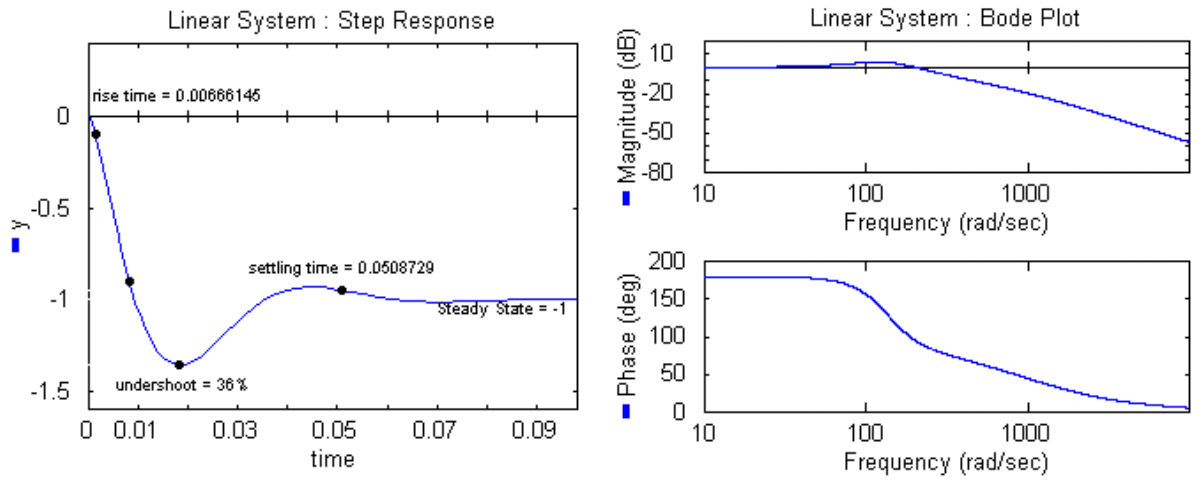

Figure 5.11: Step response of -T (left); Bode plot of -T (right).
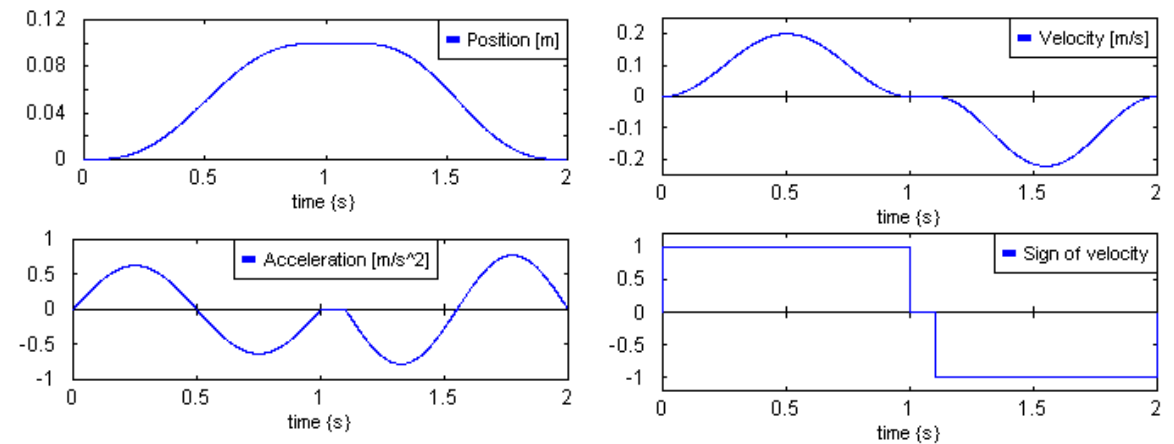

Figure 5.12: Reference inputs of the LFFC part.

The transfer function of $-T$ is determined by:

$$
-T(s)=\frac{-640 s-80900}{0.004215 s^{3}+5 s^{2}+640 s+80900} .
$$


The step response of $-T$ and the Bode plot of $-T$ are given in Fig 5.11. The reference inputs of the LFFC part are shown in Fig 5.12. The minimum support of the B-splines follows 3.12 and 3.13 is

$$
d_{\min }=0.00185[s]
$$

The maximum learning rate is:

$$
\gamma \leq 0.95
$$

For the cogging BSN, in total 500 B-splines have been defined on the position domain $(0[\mathrm{~m}], 0.1[\mathrm{~m}])$; We choose to define three B-splines on the velocity domain $\left(-0.2\left[\mathrm{~ms}^{-1}\right], 0.2\left[\mathrm{~ms}^{-1}\right]\right)$ for the viscous BSN; For the Coulomb friction BSN, three B-splines have been defined on the input domain $[-1,1]$; Three B-splines are defined on the acceleration domain $\left(-0.75\left[\mathrm{~ms}^{-2}\right], 0.75\left[\mathrm{~ms}^{-2}\right]\right)$ for the inertia BSN.

\section{Step 5: Choose the learning rate}

The learning rate is chosen relatively small, for example $\gamma=0.1$.

\subsubsection{Design of an MRAS-based LFFC}

Fig 5.13 shows the total system for testing in practice. The proposed controller consists of an MRAS-based LFFC and a separate feedback controller. The same feedback controller and the same cogging BSN are used as in case of the parsimonious LFFC introduced in Section 5.3.1. The same setpoint generator and the same state variable filter are used as in case of the MRAS-based LFFC introduced in Section 4.3.1. The Liapunov gains following from (4.28), (4.33) and (4.36) are: $p_{21}=0.3785, p_{22}=0.8359$. The adjustable parameters of the feed-forward controller are given by:

$$
\begin{gathered}
a_{m}=\frac{1}{\alpha_{a}} \sum\left[\left(p_{21} e+p_{22} \dot{e}\right) \ddot{r}\right] T_{s}+a_{m}(0) . \\
b_{m}=\frac{1}{\alpha_{b}} \sum\left[\left(p_{21} e+p_{22} \dot{e}\right) \dot{r}\right] T_{s}+b_{m}(0) . \\
d_{m}=\frac{1}{\alpha_{d}} \sum\left[\left(p_{21} e+p_{22} \dot{e}\right) \operatorname{sign}(\dot{r})\right] T_{s}+d_{m}(0) .
\end{gathered}
$$

In this design, the initial values of the feed-forward gains are chosen $a_{m}(0)=b_{m}(0)=d_{m}(0)=0$ and the adaptive gains are $\left(1 / \alpha_{a}=7000\right),\left(1 / \alpha_{b}=\right.$ $200000)$, and $\left(1 / \alpha_{d}=30000\right)$. These values are the same as used in the simulation experiments. 


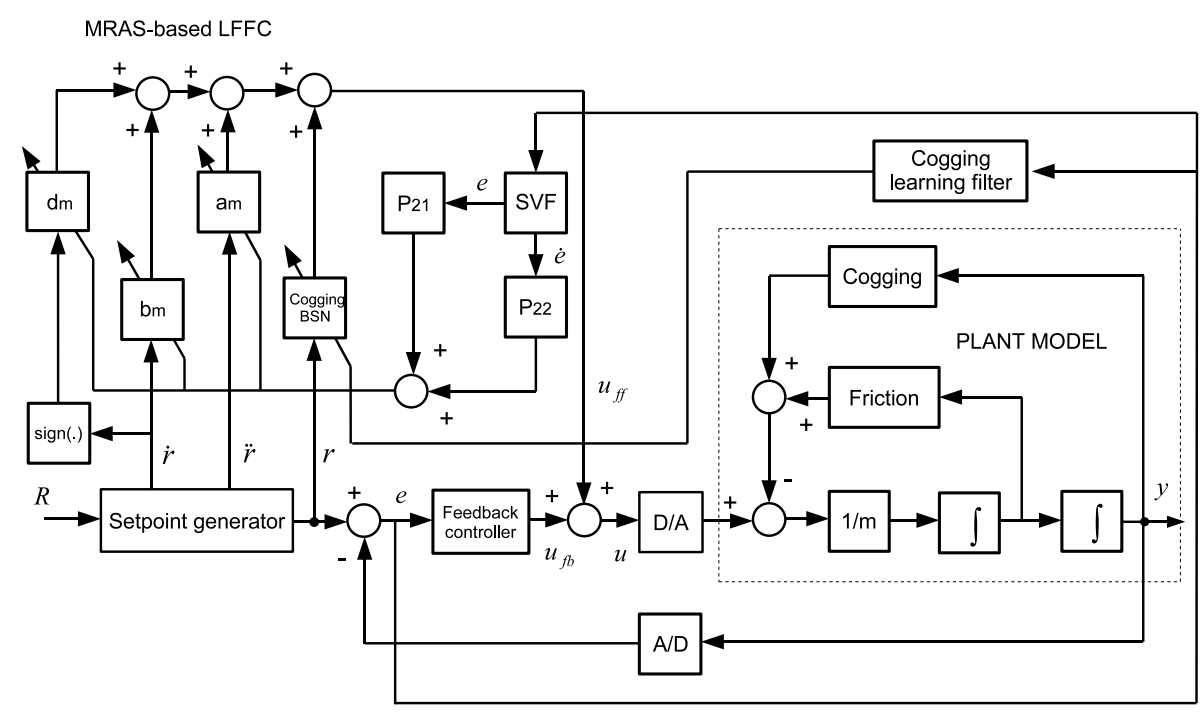

Figure 5.13: MRAS-based LFFC controlled system for each motor of the Tripod.

\subsubsection{Experimental results}

The experimental tests were carried out with only one motor moving. Fig 5.14 gives an overview of the input reference and the tracking errors for the different controllers that have been tested on the Tripod. In order to evaluate implementation of the controllers, the shape of the motion profile is purposely generated (see Fig 5.14 - top plot ). It consists of four phases; $t=0-20[\mathrm{~s}]$ and $t=36-56[\mathrm{~s}]$ : the repeating motion profiles are generated with stroke $R=0.1[\mathrm{~m}]$ and period $T=1[s] ; t=20-36[s]$ and $t=56-72[s]$ : the random motion profiles are generated.

In the first experiment only the PD controller is used. A large tracking error remains in the system due to reproducible disturbances (see Fig 5.14 - second plot). The maximum tracking error with a "slow" reference motion is 0.2 [mm] and with a "fast" reference motion it is about $0.4[\mathrm{~mm}]$. We expect, that the large tracking error with the PD controller will disappear by adding a LFFC.

In the second experiment the NN-based LFFC is added to the PD controller. With a "slow" reference motion, the reproducible disturbances are well compensated by the feed-forward part. The maximum tracking error in this situation (after 10 motions) is 0.04 [mm]. Note that convergence is fast; in about 2 strokes, the error is minimal. However, this value will be as large as 0.4 [mm] with a "fast" reference motion (see Fig 5.14 - third plot).

In the third experiment the MRAS-based LFFC is added to the PD controller. The correction signal is added directly to the output of the PD controller. First, 


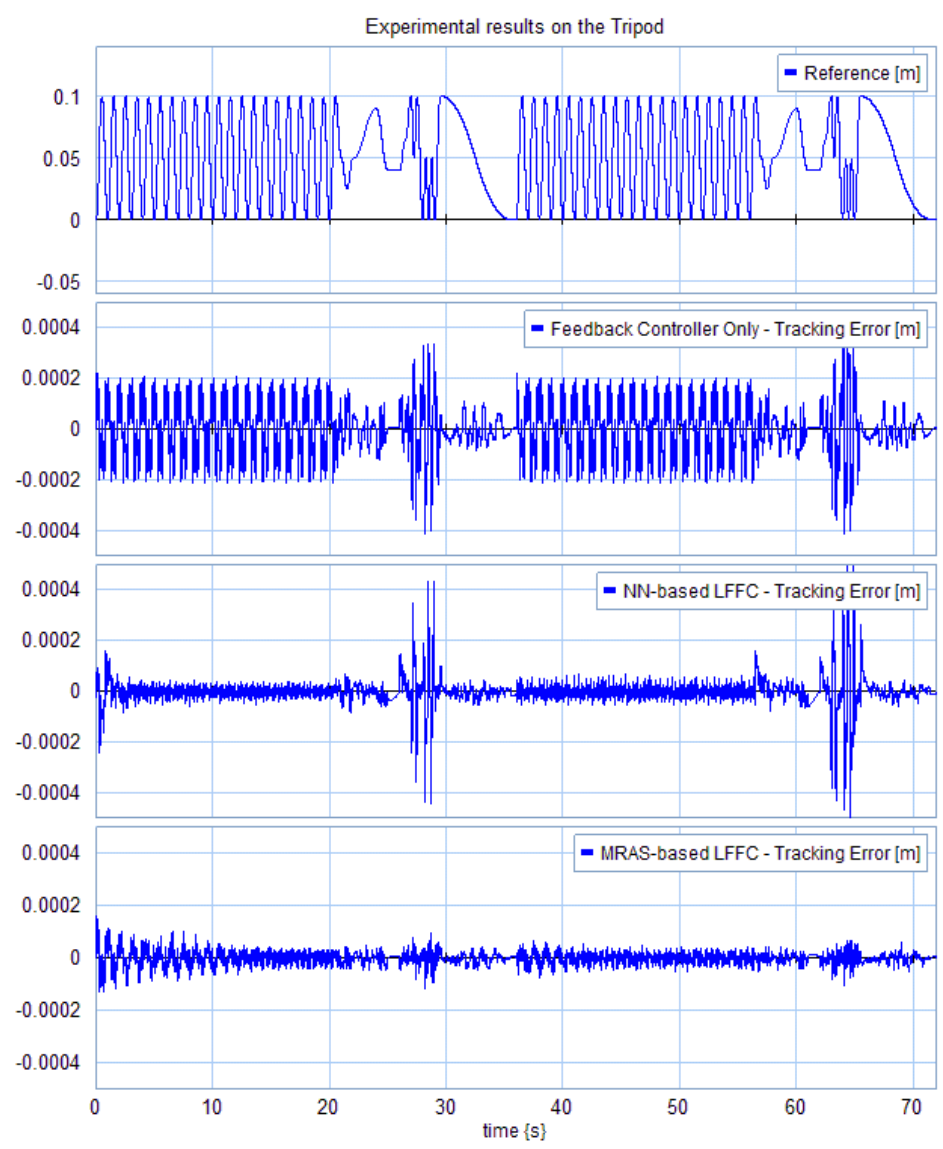

Figure 5.14: Experimental results.

with a "slow" reference motion the maximum tracking error (after 25 motions) is about 0.04 [mm]. It is observed that the influence of plant disturbances is significantly reduced. After that a "fast" motion profile is used, which results in a maximum tracking error of 0.1 [mm] (see Fig 5.14 - lowest plot). In this situation the reproducible disturbance are also well compensated by the feed-forward part. The feed-forward parameters are shown in Fig 5.15. The controlled system is stable and shows convergence in the parameters.

\section{Discussion}

1. Both simulation and experimental results are presented to verify the effectiveness of the NN-based LFFC and of the MRAS-based LFFC. These control algorithms ensure the precision and robustness of the controlled systems with respect to reproducible disturbances. They outperform the PID controller. 


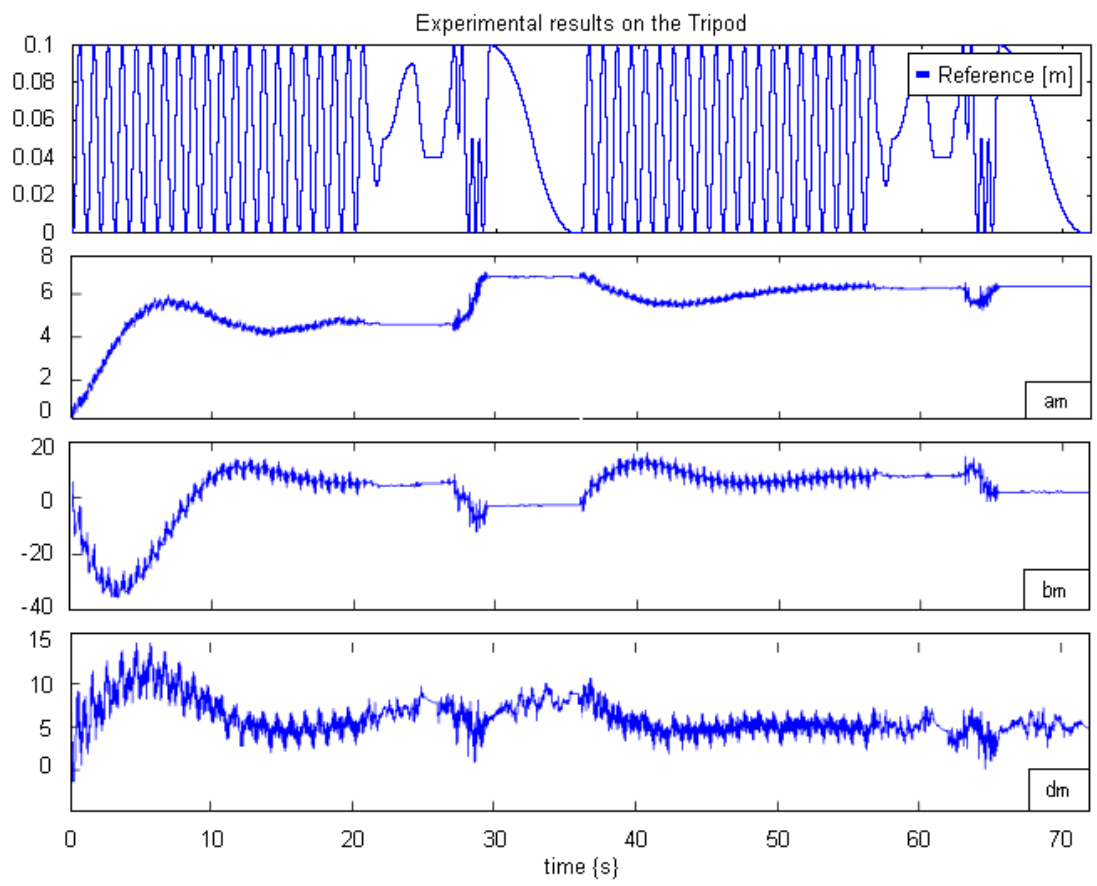

Figure 5.15: MRAS-based LFFC; Experimental parameters of the feed-forward part.

2. With a "slow" reference motion, both control algorithms show almost the same tracking errors after convergence. However, with a "fast" reference motion the MRAS-based LFFC operates much better. These results confirm an important point: when performing a 'straightforward' training (i.e., no particular sequence of carefully selected motions), the NN-based LFFC has the risk of not learning the appropriate mapping for a non-trained reference motion; with the MRAS-based LFFC, this risk is much smaller. Hence, the simplicity and 'degree' of control of the MRAS-based scheme is attractive for this reason.

3. If we would do "strategic training", the results with the NN-based LFFC would be good as well [9]. It is performed by carefully selecting a sequence of training motions such that any possible combination of reference inputs is covered. However, doing strategic training experiments is rather involved and in practice not desirable. 


\section{Chapter 6}

\section{Discussion}

\subsection{Review}

The aim of this research was to develop advanced controllers for electromechanical motion systems. A new controller is proposed in this thesis to take into account the inherent non-linear disturbances, measurement noise, and variations and uncertainties in process behavior. It consists of a Linear Quadratic Gaussian (LQG) controller and a separate supplementary MRAS-based Learning Feed-Forward Controller (LFFC). In the LQG controller, the parameter estimation is driven by the prediction error, while in the MRAS-based LFFC controller, the parameter adaptation is driven by the tracking error.

A two-degree-of-freedom optimal control law is derived, which has properties in common with both the LFFC and the LQG control laws. The responses to control problems are completely separated. In general, the LQG controller is designed to robustly stabilize the system, compensate random disturbances, and to attenuate the influence of measurement noise, whereas the adaptive feed-forward controller is used to improve the tracking performance in the presence of reproducible disturbances.

LQG is basically a combination of a Linear Quadratic Estimator (LQE) with a Linear Quadratic Regulation (LQR). The Separation principle guarantees that if a stable LQE and a stable LQR are designed for a linear time-invariant system, then a combined LQE and LQR results in a stable LQG system. The LQG design is based on a fixed mathematical model of the process. The estimator and feedback controller may be designed independently. It enables us to compromise between regulation performance and control effort, and to take into account process and measurement noise. The selection of weighting matrices $\mathrm{Q}$ and $\mathrm{R}$ has a direct effect on the system performance.

The MRAS-based LFFC aims to acquire the (stable part of the) inverse dy- 
namics of the plant. A reference model is used to generate a desired set of states. The feed-forward signal is obtained by summing the profile set-point signals multiplied by appropriate weights. On-line parameter adaptation is utilized to reduce the effect of the disturbances such as mass deviation, friction force, and cogging force resulting in a dynamic inverse of the process. With feed-forward control, the state-dependent disturbances can be compensated, before they have time to affect the system. The control action for disturbance rejection is obtained from the feedforward path output. Compared to the NN-based LFFC, the MRAS-based LFFC is simpler to implement.

In order to find the adaptive laws for the feed-forward parameters, the wellknown Liapunov stability approach can be used. The A-matrix of the reference model is used to calculate the solution $P$ of the Liapunov equation. In the adjustment laws the derivative of the error is needed. This derivative can be obtained by means of a second-order state variable filter. The parameters of this state variable filter are chosen in such a way that the parameters of the reference model can vary without the need to change the parameters of the state variable filter every time. Moreover, the SVF offers a beneficial solution to reduce the influence of the measurement noise if the noise spectrum is principally located outside the band pass of the SVFs. The adjustable parameter component has an integral component inside. This implies that even when the learning signal is corrupted by measurement noise the parameter estimates are almost noise free. This allows us using a large learning signal to shorten the setting time

With a linear approach only compensation for influence of the viscous friction is realized. It is possible to take the effects of Coulomb friction force and cogging force into account to improve the behavior of the system. This can be done by, adding extra adaptive learning components in the learning feed-forward part. For the Coulomb friction adaptive component the sign of the reference velocity is used as the input. However, the learning feed-forward that uses merely MRASbased adaptive components is unable to compensate for cogging force. A hybrid learning feed-forward part offers a solution. The cogging can be dealt with by a B-spline neural network element.

The phase shift between the actual error and the measured error at low frequencies can be ignored. However, in the high frequency range the measured error exhibits phase lag that increases with frequency. A learning filter offers an efficient solution to correct the phase lag. For the inertia adaptive component, the viscous friction adaptive component, and the Coulomb friction adaptive component in the feed-forward part the Liapunov error can be used to adapt the weights. However, for the cogging BSN, the signal from a separate learning filter output is used to adapt the weights.

The design of the LQG and the MRAS-based LFFC can be carried out separately. For the MeDe5 designs, the LQG approach suffers from the disadvantage 
in that it does not take into account the effects of reproducible disturbances, in particular Coulomb friction. The MRAS-based LFFC may be of much lower order than the process. It can respond quickly to known and predictable kinds of disturbances, but cannot do much with random disturbances. It is clear that, the combination of LQG and MRAS based LFFC control structure is shown to be superior to the two control methods when used separately. This is a robust, high-performance control scheme that combines the advantages and overcomes the disadvantages of both types of techniques. The MRAS-based LFFC can be applied to arbitrary motion profiles.

Simulation results demonstrate the potential benefits of the proposed method. In comparison to a Proportional-Integral-Derivative (PID) controller, the LQG combined with MRAS-based LFFC has the following benefits: (1) it significantly reduced the tracking errors in responding to the input reference; (2) it effectively improved the robustness to changes in plant parameters and to measurement noise; (3) it can obtain fast transient response.

For the design of a control system for a linear process using an MRAS-based LFFC it can be expected that a proper feed-forward controller signal is generated, effective for providing good tracking control performance. This is also true for some non-linearities such as Coulomb friction.

The LQG combined with MRAS-based LFFC was tested on the MeDe5. Both simulation and experimental results were presented to verify the effectiveness of the proposed control strategy. The simulation results confirm a promising tracking control capability of the proposed controller in the presence of measurement noise and plant parameter variations. From the experiment results, it is observed that the convergence of the adaptive parameters is satisfying and its control precision is better than that of the LQG controller. Moreover, the proposed controller also can obtain fast dynamic response. Thus, the LQG combined with MRAS-based LFFC is an efficient control algorithm of improving the tracking performance for electromechanical motion systems.

NN-based LFFC has been widely regarded as one of the standard control paradigms for motion systems. The use of NN-based LFFC can improve not only the disturbance rejection, but also the stability robustness of the controlled systems. One of the main drawbacks of the NN-based LFFC is the requirement that the training motions are chosen carefully, such that all possibly relevant input combinations are covered. This requirement may be quite restrictive in practical applications. To overcome such problem, the use of MRAS-based LFFC can be applied. Compared to the NN-based LFFC, the MRAS-based LFFC is simpler to implement. The resulting control laws are simple and thus interesting for use in practical applications. By implementing both controllers on the Tripod setup, the performances of each method are compared. The experimental results show that both control algorithms reach almost the same tracking error after convergence 
and are superior to the PID controller. However, after convergence the MRASbased LFFC is able to generate a much better feed-forward control and hence obtain about a 5 times smaller maximum tracking error than the NN-based LFFC with an untrained reference motion.

\subsection{Conclusions}

The conclusions that can be drawn from this research concerning advanced controller for electromechanical motion systems are:

1. In the design of an electromechanical motion system, the following typical factors have to be considered: (a) reduction of the effect of reproducible disturbances; (b) attenuation of measurement noise; and (c) uncertainties and variations in plant behavior. It is difficult to control such a system with feedback alone to obtain high performance and robustness.

2. A PID controller has some limitations. In order to cope with the fact that the parameters of the system being controlled are slowly time-varying and/or uncertain, and the presence of reproducible disturbances, high PID gains are required. However, having large gains can lead to system instability. In general, for the PID control design, a compromise has to be made between performance and robust stability.

3. In the LQG design the main issue is to trade-off attenuation of the process disturbances and the fluctuations created by measurement noise that is injected in the system due to the feedback. However, the LQG algorithm may fail to ensure closed-loop stability if the variations or/and uncertainties are large enough.

4. When the parameters of the system are unknown or time varying, an adaptive control algorithm could be used. The stability of the closed-loop system can be guaranteed by the Lyapunov theory. Direct MRAS offers a potential solution to reduce the tracking errors. However, this control algorithm may fail to be robust to measurement noise. Indirect MRAS offers an effective solution to improve the control performance in the presence of parametric uncertainty and measurement noise. However, a small tracking error cannot always be obtained. In general, by using a single degree of freedom controller, small tracking errors and good measurement noise rejection properties cannot be implemented simultaneously.

5. Since most practical motion control systems are subject to reproducible disturbances, NN-based LFFC is used to address this problem. The NN-based 
LFFC can be designed on the basis of qualitative knowledge of the plant and the disturbances. If a set of the reproducible disturbances need to be rejected, a corresponding set of feed-forward signals is created, with each individual feed-forward signal designed to reject only one of the known reproducible disturbances. When the feedback signal is used to learn the statedependent effects, learning filter can be used to correct the phase lag. Simulation and experimental results show that the NN-based LFFC can achieve a low tracking error compared with conventional control methods. This approach can be effectively used for non-repetitive motions. NN-based LFFC can achieve good disturbance, but the performance is limited by measurement noise. Furthermore, it is not clear how the learning rule of LFFC is obtained in the stability sense.

6. LQG combined with MRAS-based LFFC offers a potential solution to deliver more accurate and high overall performance in the presence of all the preceding issues. In comparison to NN-based LFFC positive points of MRAS-based LFFC are its simplicity, and its flexibility against variations in the motion profile to be realized. Moreover, an important difference is that the stability of the MRAS-based LFFC is analyzed by the Liapunov theory. In other words, the stability properties of the MRAS-based LFFC are better understood. The design of the LQG and the MRAS-based LFFC can be carried out separately. We investigated the effect of the controller from the simulation results and the experimental results. Compared to the case with LQG controller only, the proposed controller, for instance, can do the following: (1) Improve the transient behavior of the system; (2) Decrease the sensitivity to plant parameter changes; (3) Eliminate steady-state errors; and (4) Decrease the influence of load disturbances and measurement noise. The simulation results show that the proposed method exhibits better performances than linear or other controllers, especially when large plant parameter variations and measurement noise occur. Strong properties achieved via the proposed method confirm that LQG combined with MRAS-based LFFC is an attractive approach for controlling electromechanical motion systems.

\subsection{Recommendations for future work}

1. In Chapter 4, as can be seen in Fig 4.11, after a short time the parameters $a_{m}$, $b_{m}$, and $c_{m}$ in the feed-forward part converse quickly to stationary values. They denote the characteristic of the process model and could be used for the LQG design. The optimal steady-state filter gain $L$ in the LQE and the feedback gain $K$ in the LQR can be determined based on the parameters of 
the feed-forward part, which follows continuously the process at different load conditions. This will result in "an adaptive LQG combined with the MRAS-based LFFC" like in the control structure of Fig 2.22. In addition, the derivative of the error could be obtained from the LQE instead of the SVF.

2. In Chapter 5, the experiments were carried out with a single linear motor, which was not operated together with the other motors of the Tripod. The LFFC only had to learn the characteristics of the single motor. In the next design and experiments, all three motors have to move simultaneously. Apart from the motor characteristics, the dynamic coupling from the other motors has to be learned. 


\section{Appendix A: The MeDe5}

As was introduced in Chapter 1, the MeDe5 has a slider, which is connected to the motor inertia with an elastic belt. The Motor and slider are placed on an elastic frame (see Fig 1). If all these springs and masses are taken into account, the MeDe5 can be considered as a 6th order system [14].

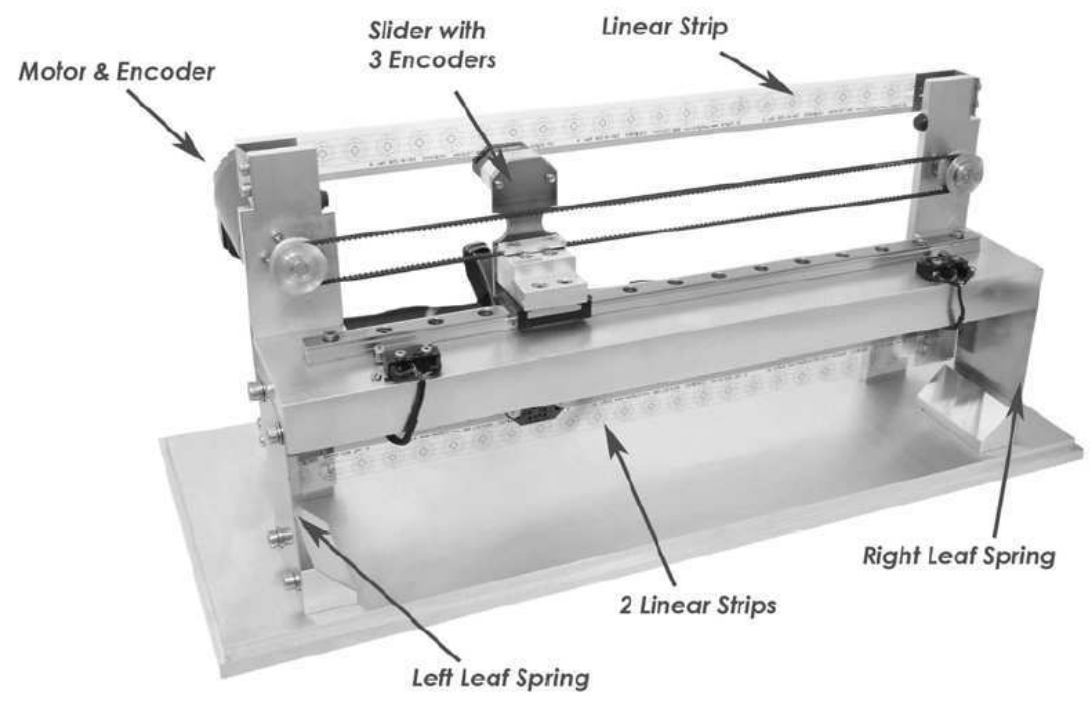

Figure 1: Mechanical setup of the MeDe5

The $6^{\text {th }}$ order linear plant model is described in state space form as given in Formulas A.2 and A.3. The abbreviations used in these formulas are explained in Table 1.

$$
\begin{gathered}
\left\{\begin{array}{l}
\dot{x}=A x+B u \\
y=C x+D u .
\end{array}\right. \\
{\left[\begin{array}{c}
\dot{v}_{L} \\
\dot{x}_{L} \\
\dot{v}_{F} \\
\dot{x}_{F} \\
\dot{v}_{M} \\
\dot{x}_{M}
\end{array}\right]=\left[\begin{array}{cccccc}
\frac{-\left(d_{B}+d\right)}{m} & 0 & \frac{d}{m} & 0 & \frac{d_{B}}{m} & \frac{k_{B}}{m} \\
1 & 0 & 0 & 0 & 0 & 0 \\
\frac{d}{m_{F}} & 0 & \frac{-\left(d_{F}+d\right)}{m_{F}} & \frac{k_{F}}{m_{F}} & 0 & 0 \\
0 & 0 & -1 & 0 & 0 & 0 \\
\frac{d_{B}}{m_{I}} & 0 & 0 & 0 & \frac{-d_{B}}{m_{I}} & \frac{-k_{B}}{m_{I}} \\
-1 & 0 & 0 & 1 & 0 & 0
\end{array}\right]\left[\begin{array}{c}
v_{L} \\
x_{L} \\
v_{F} \\
x_{F} \\
v_{M} \\
x_{M}
\end{array}\right]+\left[\begin{array}{c}
0 \\
0 \\
\frac{-k_{m}}{m_{F}} \\
0 \\
\frac{k_{m}}{m_{I}} \\
0
\end{array}\right] F}
\end{gathered}
$$




$$
y=\left[\begin{array}{llllll}
0 & 1 & 0 & 0 & 0 & 0 \\
0 & 0 & 0 & 1 & 0 & 0 \\
0 & 0 & 0 & 0 & 0 & 1
\end{array}\right]\left[\begin{array}{l}
v_{L} \\
x_{L} \\
v_{F} \\
x_{F} \\
v_{M} \\
x_{M}
\end{array}\right]+\left[\begin{array}{l}
0 \\
0 \\
0
\end{array}\right] F
$$

\begin{tabular}{|c|l|c|l|}
\hline$v_{L}$ & state1: Velocity of the load & $d_{B}$ & Damping of the belt \\
\hline$x_{L}$ & state2: Position of the load & $k_{B}$ & Stiffness of the belt \\
\hline$v_{F}$ & state3: Velocity of the frame & $d_{F}$ & Damping in the frame \\
\hline$x_{F}$ & state4: Position of the frame leaf springs & $k_{F}$ & Stiffness of the frame \\
\hline$v_{M}$ & state5: Velocity of motor inertia & $d$ & Damping in the damper \\
\hline$x_{M}$ & state6: Position of the belt & $m$ & Mass of the load \\
\hline$k_{m}$ & Motor constant & $m_{I}$ & Mass of the motor inertia \\
\hline$F$ & Applied force on the process & $m_{F}$ & Mass of the frame \\
\hline
\end{tabular}

Table 1: Explanation of the variables and parameters used in Formulas A.2 and A.3

When the motor-inertia and the belt-flexibility are not taken into account (see Fig 2), a fourth order approximation model is obtained with a state space description as given in Formulas A.4 and A.5.

$$
\begin{aligned}
& {\left[\begin{array}{c}
\dot{v}_{L} \\
\dot{x}_{L} \\
\dot{v}_{F} \\
\dot{x}_{F}
\end{array}\right]=\left[\begin{array}{cccc}
\frac{-d}{m} & 0 & \frac{d}{m} & 0 \\
1 & 0 & 0 & 0 \\
\frac{d}{m_{F}} & 0 & \frac{-\left(d_{F}+d\right)}{m_{F}} & \frac{k_{F}}{m_{F}} \\
0 & 0 & -1 & 0
\end{array}\right]\left[\begin{array}{c}
v_{L} \\
x_{L} \\
v_{F} \\
x_{F}
\end{array}\right]+\left[\begin{array}{c}
\frac{k_{m}}{m} \\
0 \\
\frac{-k_{m}}{m_{I}} \\
0
\end{array}\right] F,} \\
& y=\left[\begin{array}{llll}
0 & 1 & 0 & 0 \\
0 & 0 & 0 & 1
\end{array}\right]\left[\begin{array}{l}
v_{L} \\
x_{L} \\
v_{F} \\
x_{F}
\end{array}\right]+\left[\begin{array}{l}
0 \\
0
\end{array}\right] F,
\end{aligned}
$$

where

$$
\begin{gathered}
A_{c 4}=\left[\begin{array}{cccc}
\frac{-d}{m} & 0 & \frac{d}{m} & 0 \\
1 & 0 & 0 & 0 \\
\frac{d}{m_{F}} & 0 & \frac{-\left(d_{F}+d\right)}{m_{F}} & \frac{k_{F}}{m_{F}} \\
0 & 0 & -1 & 0
\end{array}\right]=\left[\begin{array}{cccc}
-10 & 0 & 10 & 0 \\
1 & 0 & 0 & 0 \\
3.75 & 0 & -11.25 & 7500 \\
0 & 0 & -1 & 0
\end{array}\right] . \\
B_{c 4}=\left[\begin{array}{c}
\frac{k_{m}}{m} \\
0 \\
\frac{-k_{m}}{m_{I}} \\
0
\end{array}\right]=\left[\begin{array}{c}
19 \\
0 \\
-7.125 \\
0
\end{array}\right], C_{c 4}=\left[\begin{array}{llll}
0 & 1 & 0 & 0 \\
0 & 0 & 0 & 1
\end{array}\right], D_{c 4}=\left[\begin{array}{l}
0 \\
0
\end{array}\right] .
\end{gathered}
$$




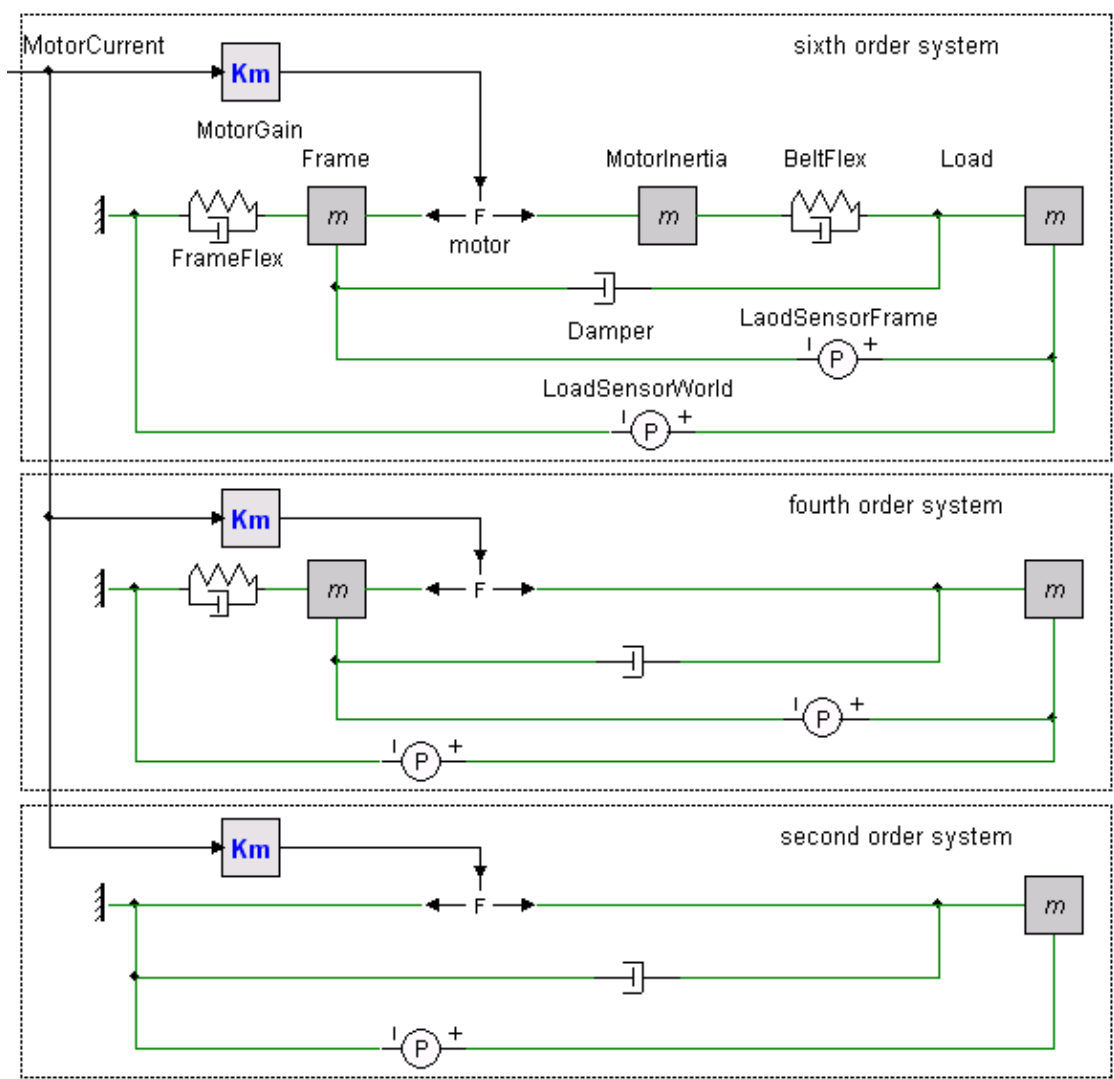

Figure 2: Comparison of 6th, 4th, and 2nd-order systems

A second order approximation model is obtained when the frame-flexibility is continuously not taken into account (see Fig 2). Its state space form is given in Formulas A.8 and A.9.

$$
\begin{gathered}
{\left[\begin{array}{c}
\dot{v}_{L} \\
\dot{x}_{L}
\end{array}\right]=\left[\begin{array}{cc}
\frac{-d}{m} & 0 \\
1 & 0
\end{array}\right]\left[\begin{array}{c}
v_{L} \\
x_{L}
\end{array}\right]+\left[\begin{array}{c}
\frac{k_{m}}{m} \\
0
\end{array}\right] F .} \\
y=\left[\begin{array}{ll}
0 & 1
\end{array}\right]\left[\begin{array}{c}
v_{L} \\
x_{L}
\end{array}\right]+[0] F .
\end{gathered}
$$

where

$$
\begin{gathered}
A_{c 2}=\left[\begin{array}{cc}
\frac{-d}{m} & 0 \\
1 & 0
\end{array}\right]=\left[\begin{array}{cc}
-10 & 0 \\
1 & 0
\end{array}\right], B_{c 2}=\left[\begin{array}{c}
\frac{k_{m}}{m} \\
0
\end{array}\right]=\left[\begin{array}{c}
19 \\
0
\end{array}\right] . \\
C_{c 2}=\left[\begin{array}{ll}
0 & 1
\end{array}\right], D_{c 2}=[0] .
\end{gathered}
$$


When we mention the nonlinear friction term of the Damper element then:

$$
\left[\begin{array}{c}
\dot{v}_{L} \\
\dot{x}_{L}
\end{array}\right]=\left[\begin{array}{cc}
-\frac{d}{m} & 0 \\
1 & 0
\end{array}\right]\left[\begin{array}{l}
v_{L} \\
x_{L}
\end{array}\right]+\left[\begin{array}{c}
-\frac{d_{c}}{m} \operatorname{sign}\left(v_{L}\right) \\
0
\end{array}\right]+\left[\begin{array}{c}
\frac{k_{m}}{m} \\
0
\end{array}\right] F
$$

where $d_{c}$ is the Coulomb parameter of the Damper element.

Since discrete controllers will be implemented in the MeDe5 setup, discretization of these state matrices is required. This has been done by the C2DMcommand in Matlab, assuming a zero-order-hold discretization process and a sample frequency of $1[\mathrm{KHz}]$, the corresponding discrete state matrices are:

$$
\begin{gathered}
A_{d 4}=\left[\begin{array}{cccc}
1.0000 & 0.0000 & -0.0100 & 0.0000 \\
0.0010 & 1.0000 & 0.0000 & 0.0000 \\
0.0075 & 7.5000 & 0.9787 & -7.5000 \\
0.0000 & 0.0000 & 0.0010 & 1.0000
\end{array}\right], B_{d 4}=\left[\begin{array}{l}
0.0190 \\
0.0000 \\
0.0261 \\
0.0000
\end{array}\right] \\
C_{d 4}=\left[\begin{array}{cccc}
0 & 1 & 0 & 0 \\
0 & 0 & 0 & 1
\end{array}\right] \\
A_{d 2}=\left[\begin{array}{cc}
0.990 & 0.000 \\
0.001 & 1.000
\end{array}\right], B_{d 2}=\left[\begin{array}{l}
0.019 \\
0.000
\end{array}\right] \\
C_{d 2}=\left[\begin{array}{ll}
0 & 1
\end{array}\right], D_{d 4}=\left[\begin{array}{l}
0 \\
0
\end{array}\right], D_{d 2}=[0]
\end{gathered}
$$




\section{Bibliography}

[1] Ahn, H. S., Chen, Y. Q., and Dou, H., 2005, State-Periodic Adaptive Compensation of Cogging and Coulomb Friction in Permanent Magnet Linear Motors, 2005 American Control Confrence, Portland, OR, USA.

[2] Anderson, Brian D. O., Moore, John B., 1979, Optimal Filtering, Information and System Sciences Series, Thomas Kailath Editor, Prentice Hall Electrical Engineering Series, ISBN 0-13-638122-7.

[3] Aström, K. J., Hagglund, T., 1995, PID controllers: Theory, Design and Tuning, Instrument Society of America, Research Triangle Park, NC, USA.

[4] Aström, K. J., Wittenmark, B., 1989, Adaptive Control, Lund Institute of Technology, Addison-Wesley Publishing Company, TJ217.A67 1989.

[5] Aström, K. J., Wittenmark, B., 1997, Computer-Controlled Systems - Theory and Design, Third Edition, Prentice Hall Information and System sciences Series, Prentice Hall, Upper Saddle River, New Jersey 07458, ISNB 0-13314899-8.

[6] Berghuis, H., 1993, Model-Based Robot Control: from Theory to Practice, $\mathrm{PhD}$ thesis, University of Twente, Enschede,The Netherlands.

[7] Coelingh, H. J., 2000, Design Support for Motion Control Systems: a mechatronic approach, $\mathrm{PhD}$ thesis, University of Twente, Enschede, The Netherlands.

[8] Controllab Products B.V, 20-Sim-software version 3.6, 2006 (http://www.20sim.com).

[9] de Kruif, Bas J., 2004, Function Approximation for Learning Control: a key sample based approach, $\mathrm{PhD}$ thesis, University of Twente, Enschede, The Netherlands. 
[10] de Kruif, Bas J., de Vries, Theo J. A., 2003, Phase Correction for Learning Feed-Forward Control, Proceeding of the 2003 IEEE/ASME, International Conference on Advanced Intelligent Mechatronics.

[11] de Vries, Theo J. A., Idema, L. J., and Velthuis, Wubbe J. R., 1998, Parsimonious Learning Feed-Forward Control, Proceeding 6th European Symposium on Artificial Neural Networks ESANN (Bruges, Belgium), M. Verleysen (editor), ISBN 2-9600049-8-1.

[12] de Vries, Theo J. A., Velthuis, Wubble J. R., and van Amerongen, J., 2000, Learning Feed-Forward Control: A survey and Historical Note, Proc. 1th IFAC Conf. on Mechatronic Systems (Darmstadt, Germany, 18-20 Sept.2000), R. Tsermann (ed.), VDI/VDE Gesellschaft Mess-und Automatiserungstechnick GMA, Dusseldorf, Germany.

[13] de Vries, Theo J. A., Velthuis, Wubbe J. R., and Idema, L. J., 2001, Application of Parsimonious Learning Feed-Forward Control to Mechatronic Systems, IEE Pro. Control Theory Appl, Vol. 148, No. 4, July 2001.

[14] Dirne, H., Demonstrator of Advanced Controller, Master thesis, University of Twente, The Netherlands, May 2005.

[15] Dorf, R. C., Bishop, R. H., 2005, Mordern Control Systems, Tenth Edition, Pearson Educational International, Pearson Prentice Hall, Upper Saddle River, NJ 07458, ISBN: 0-13-127765-0.

[16] Eglence, M., Design and Realization of a Safe Control System for a Parallel Manipulator, Master thesis, University of Twente, The Netherlands, June 2003.

[17] Ge, S. S., Lee, T. H., and Ren, S. X., 2001, Adaptive Friction Compensation of Servo Mechanisms, International Journal of Systems Science, volume 32, number 4, pages 523-532.

[18] Hesselink, R. O., MRAS-based Learning Feed-Forward Control, Master thesis, University of Twente, The Netherlands, November 2006.

[19] Kawato, M., 1990, Feedback-Error-Learning Neural Network for Supervised Motor Learning, 365-372, Advanced Neural Computers, Amsterdam, The Netherlands, Elsevier Science Publishers B.V.

[20] Lammerts, Ivonne M. M., 1993, Adaptive Computed Reference Computed Torque Control of Flexible Manipulators, $\mathrm{PhD}$ thesis, Eindhoven University of Technology, Eindhoven, The Netherlands. 
[21] Landau, I. D., Lozano, R., and Saad, M. M'., 1998, Adaptive Control, Communications and Control Engineering, Springer-Verlag London Limited, printed in Great Britain, ISBN 354076187x.

[22] Landau, Y. D., 1979, Control and Systems Theory - Adaptive Control - The Model Reference Approach, Marcel Dekker, INC, ISBN 0-8247-6548-6.

[23] Maciejowski, J. M., 2003, Predictive Control with Constrains, Prentice Hall, ISBN 0201398230 PPR.

[24] Miller, W. T., Sutton, R. S., and Werbos, P. J., 1995, Neural Networks for Control, The MIT Press Cambridge, Massachustts London, England, ISBN 0-262-13261-3 (HB), 0-262-63161-X (PB).

[25] Moore, K. L., 1993, Iterative learning control for deterministic systems, Advances in Industrial Control. Springer - Verlag.

[26] Ng, G. W., Application of Neural Networks to Adaptive Control of Nonlinear Systems, 1997, Control Systems Center Series, Research Studies Press LTD, Jonh Wiley and Sons INC, ISBN 086380214.

[27] Nguyen, C. D., Dao, P. B., Phan, M. X., 2006, A Review of Classical and Advanced Controllers, HUT conference of Automatic, Ha noi, 2006.

[28] Nguyen, C. D., Dao, P. B., Phan, M. X., MRAS-based Controllers, Vietnamese conference of Mechatronics, Ha noi, 2006.

[29] Nguyen, C. D., Dao, P. B., de Vries, Theo J. A., van Amerongen, J., 2007, Learning Feed-Forward Controller for Electromechanical Motion Systems, Vietnamese conference of Mechanic, Ha noi, 2007.

[30] Nguyen, C. D., Dao, P. B., de Vries, Theo J. A., van Amerongen, J., An Advanced Controller Applied to Electromechanical Motion Systems, Vietnamese conference of Mechanic, Ha noi, 2007.

[31] Nguyen, H. T., Prasad, N. R., Walker, C. L., and Walker, E. A., 2003, A First Course in Fuzzy and Neural Control, Chapman and Hall/CRC, A CRC Press Company.

[32] Nogaard, M., Ravn, O., Poulsen, N. K., and Hansen, L. K., 2000, Neural Network for Modeling and Control of Dynamic Systems, Advanced Textbooks in Control and Signal Processing, ISBN 1- 85233-227-1 Springer Verlag London. 
[33] Ogata, K., 1997, Modern Control Engineering, Third Edition, Prentice Hall International, Simon and Schuster/A Viacom Company, Upper Saddle River, NJ 07458.

[34] Otten, G., de Vries, Theo J. A., van Amerongen, J., Rankers, A. M., and Gaal, E. W., 1997, Linear Motor Motion Control Using a Learning FeedForward Controller, IEEE/ASME Transactions on Mechatronics, Vol. 2, No. 3, 1997.

[35] Paraskevopoulos, P. N., 1996, Digital Control Systems, Prentice Hall, Mathematical Composition Setters Ltd, ISBN 0-13-341876-6.

[36] Stramigioli, S., Intelligent Control Course 2004, Sheets of the lecture on Self Tuning Regulators, University of Twente, The Netherlands, 2004.

[37] Sunan, H., Kiong, T. K., and Heng, L. T., 2002, Applied Predictive Control, Advanced in Industrial Control, Springer, ISBN 18523333383.

[38] Tomizuka, M., 1987, Zero Phase Error Tracking Algorithm for Digital control, Journal of Dynamic Systems, Measurement, and Control, vol.109, pp.65-68.

[39] van Amerongen, J., de Vries, Theo J. A., and Coelingh, E., Computer Support for Mechatronic Control Design, The invited session on 'Computer Support for Mechatronics System Design' at the International Conference on Advanced Mechatronics, Okayama, Japan, 3-5 August 1998.

[40] van Amerongen, J., Intelligent Control (part 1)-MRAS, Lecture notes, University of Twente, The Netherlands, March 2004.

[41] van Amerongen, J., 2004, 15 Years of Experience with Mechatronics Research and Education, The 9th Mechatronics Forum International Conference, Ankara, Turkey.

[42] van Amerongen, J., de Vries, Theo J. A., Digital Control Engineering, University of Twente, The Netherlands, May 2005.

[43] van Amerongen, J., An MRAS-based learning feed-forward controller, Mechatronics 2006 - 4th IFAC-Symposium on Mechatronic Systems Heidelberg, Germany, September 12th-14th, 2006, pp. 6, 2006.

[44] van Twillert, H., Demonstrator of Advanced Controllers, Adaptive control configurations, Master thesis, University of Twente, The Netherlands, August 2005. 
[45] Velthuis, Wubbe J. R., 2000, Learning Feed-Forward Control: Theory, Design, and Applications, PhD thesis, University of Twente, Enschede, The Netherlands.

[46] Velthuis, Wubbe J. R., de Vries, Theo J. A., and van Amerongen, J., 2000, Design procedure for a Learning Feed-Forward Controller, Proc. 1th IFAC Conf. on Mechatronic Systems (Darmstadt, Germany, 18-20 Sept.2000), R. Tsermann (ed.), VDI/VDE Gesellschaft Mess-und Automatiserungstechnick GMA, Dusseldorf, Germany.

[47] Verwoerd, M., 2005, Iterative Learning Control - a critical review, $\mathrm{PhD}$ thesis, University of Twente, Enschede, The Netherlands.

[48] Wassink, M., Design of an Indirect Self Tuning Regulator simulation model for 20-sim, Report nr. 045 CE 2005, University of Twente, The Netherlands, 2005.

[49] Yao, B., 1997, High performance adaptive robust control of nonlinear systems: a general framework and new schemes, Proceeding of the IEEE Conference on Decision and Control, p.2489-94.

[50] Yao, B., Tomizuka, M., 1996, Smooth robust adaptive sliding mode control of robot manipulators with guaranteed transient performance. Trans J Dyn Syst, Meas, Control; 118(4):764-775.

[51] Yao, B., Xu, L., 2000, Adaptive robust motion control of linear motors for precision manufacturing, Proceeding of the Mechatronics 2002, S09574158.

[52] Zbikowski, R., Hunt, K. J., 1996, Neural Adaptive Control Technology, World Scientific Series in Robotics and Intelligent Systems - Vol.15, ISBN 9810225571. 


\section{Acknowledgments}

The research presented in this thesis will have never been accomplished without the help and support from many people. I would like to express my thanks to all of them.

First of all, I would like to express my gratitude to my promotor, Prof.dr.ir. Job van Amerongen, who brought me to the Control Engineering group. At the beginning, I had no background knowledge as many $\mathrm{PhD}$ students in the $\mathrm{CE}$ group. I went to his room every Wednesday, asking questions, discussing ideas. He suffered a lot from my English. He supported me all the time, giving me the necessary encouragements after each small success, which I achieved. With his extensive knowledge on Mechatronics, Intelligent Control, and Modeling and Simulation, he helped me a lot in solving problems. His comments on my work were always short but meaningful. I benefited a lot from his remarks. Thank you very much Prof. Job, I cannot express my appreciation enough to you.

Next, I wish to thank my assistant-promotor, Dr.ir. T.J.A. de Vries, for his guidance, discussions, and the Dutch translation of the summary. His criticisms about our works were strict and helpful. Every paragraph in this thesis became precise and clear after his corrections and comments.

I am thankful to the Vietnamese Government for financial support. Thanks to all members of the 322- project from the MOET for their management and service.

I would like to thank all the committee members for their careful reading and constructive comments to my thesis.

I would like to thank all the members of the CE group for a pleasant working atmosphere. I am very grateful to Carla for her useful helps with all documents relating to the period of my studying in the Netherlands. My thanks are expressed to Dr.ir. Peter, Dr.ir. Jan, Dr.ing. Raffaella, Dr. Maarten, Prof.dr.ir. Paul, Prof.dr.ir Stefano, Frank, Peter, Paul, and Christian for their supports. I thank Gerben, Marcel, and Alfred for their technical help. The other colleagues: Michel, Marcel, Aditya, Gijs , Martijin, Wei, Martin, Edwin, and Oscar made the wonderful time for me. I thank Tim for his working on the Tripod setup.

I would like to express my gratitude to all people of the Thainguyen University 
of Technology for their supports. My acknowledgments are also sent to Prof. dr. Vo Quang Lap - my supervisor for my bachelor and also master studies. I wish to thank Prof.dr. Nguyen Dang Binh for his encouragements and supports. My thanks and regards are expressed to Cao Thanh Long, Vu Ngoc Pi, Nguyen Van Du, Nguyen Tien Hung, Le Quoc Phong, and Nguyen Duc Thang for their cheerful friendship and help during the years.

My thanks are also sent to all members of the K15 I company for their useful helps to my family.

To my Vietnamese friends in Twente, Delft, Groningen, Leiden, Hanoi, and Thainguyen. I can only mention the names of some of them here: Nhon-Loi (Hanoi), Giang-Linh (Thainguyen), Kim-Van Anh, Ha-Hanh, Hien-Nhu, Tu-An, Trung-Thanh, Giang-Chi, Duy-Chi, Thang-Mai, Phuong-Ha, Ha-Huong, HoaHuong, Nhung, Phong, Chau, Nhi, Minh, So, Hanh (ITC), Minh and Khuong (Groningen), Hoan (Delft), Sam (Leiden). Thank all of you, my friends!

My most grateful worlds are expressed to my parents, my sister Canh-Ty and brothers Hung-Tam and Cuong-Cham for their whole life support and love that they have given me.

I thank my wife Linh for her patience love and sacrifices, which she has done for me, my daughter Thanh Van and my son Duy An.

Enschede, $10^{\text {th }}$ of March, 2008.

Nguyen Duy Cuong 


\section{About the Author}

Nguyen Duy Cuong was born on May 9th, 1962 in Camduong - Laocai, a small town in the north of Vietnam. After completing his secondary education in 1979, he started his bachelor study in Electrical Engineering at the Thainguyen University of Technology in Vietnam. In 1984, he obtained his bachelor degree and worked as a lecturer at the faculty of Electrical Engineering of the same university in the field of Electronic Technique. In 2001, he obtained his M.Sc. degree with a thesis on automatic control of an industrial furnace. In 2004, he was granted a scholarship from the Vietnamese Government through the 322 project and started his Ph.D. position at the Control Engineering group, University of Twente, The Netherlands, under the supervision of Prof.dr.ir. Job van Amerongen and Dr.ir. Theo.J.A. de Vries. He has successfully completed his $\mathrm{PhD}$ research on Advanced Controllers for Electromechanical Motion Systems. 